NBER WORKING PAPER SERIES

\title{
HOW NEWS AND ITS CONTEXT DRIVE RISK AND RETURNS AROUND THE WORLD
}

\author{
Charles W. Calomiris \\ Harry Mamaysky \\ Working Paper 24430 \\ http://www.nber.org/papers/w24430 \\ NATIONAL BUREAU OF ECONOMIC RESEARCH \\ 1050 Massachusetts Avenue \\ Cambridge, MA 02138 \\ March 2018
}

We gratefully acknowledge support from the Program for Financial Studies and the Bank of England, and excellent research assistance from Yong Wang, Minchen Zheng, and Sirui Wang. We thank the Thomson Reuters Corp. for graciously providing the data that was used in this study. For helpful comments we thank Kent Daniel, Robert Hodrick, Leif-Anders Thorsrud, Diego Garcia, an anonymous referee, and seminar participants at Catholic University of Chile, the 2016 RIDGE/ Banco Central del Uruguay Workshop on Financial Stability, the AlphaSimplex Group, the 2017 News \& Finance Conference at Columbia, Chapman University, Villanova University, Ohio State University, the Federal Reserve Bank of Kansas City, Arizona State University, the Global Risk Institute, and the University of Colorado. The views expressed herein are those of the authors and do not necessarily reflect the views of the National Bureau of Economic Research.

NBER working papers are circulated for discussion and comment purposes. They have not been peer-reviewed or been subject to the review by the NBER Board of Directors that accompanies official NBER publications.

(C) 2018 by Charles W. Calomiris and Harry Mamaysky. All rights reserved. Short sections of text, not to exceed two paragraphs, may be quoted without explicit permission provided that full credit, including $\odot$ notice, is given to the source. 
How News and Its Context Drive Risk and Returns Around the World

Charles W. Calomiris and Harry Mamaysky

NBER Working Paper No. 24430

March 2018

JEL No. G12,G14,G15

\begin{abstract}
$\underline{\text { ABSTRACT }}$
We develop a classification methodology for the context and content of news articles to predict risk and return in stock markets in 51 developed and emerging economies. A parsimonious summary of news, including topic-specific sentiment, frequency, and unusualness (entropy) of word flow, predicts future country-level returns, volatilities, and drawdowns. Economic and statistical significance are high and larger for year-ahead than monthly predictions. The effect of news measures on market outcomes differs by country type and over time. News stories about emerging markets contain more incremental information. Out-of-sample testing confirms the economic value of our approach for forecasting country-level market outcomes.
\end{abstract}

\author{
Charles W. Calomiris \\ Columbia Business School \\ 3022 Broadway Street, Uris Hall \\ New York, NY 10027 \\ and NBER \\ cc374@columbia.edu \\ Harry Mamaysky \\ Columbia Business School \\ Uris Hall \\ 3022 Broadway \\ New York, NY 10027 \\ hm2646@columbia.edu
}

An online appendix is available at http://www.nber.org/data-appendix/w24430 


\section{Introduction}

What is news and how is it associated with changes in stock market returns and risks? This is a fundamental question in asset pricing and has been the subject of decades of research (for example, Fama et al. 1969, Roll 1984). Recently, financial economists have brought new tools to bear on this question, including the analysis of the relationships between market outcomes for individual stocks or U.S. stock market indexes and various aspects of the words that appear in newspaper articles and other textual sources (for example, Tetlock 2007, 2010, 2011, Tetlock et al. 2008, Garcia 2013). This literature is still in its infancy, but already it has become apparent that it is possible to quantify salient aspects of word flow and link these measures to market outcomes. Moreover, the information contained in these textual analyses not only is associated with changes in returns and risk, but can provide incremental predictive value over and above other variables that are associated with changes in returns and risk (such as "value" or "momentum" measures, or measures of economic activity or changes in related asset prices, such as exchange rates or interest rates).

The promising early work in the literature linking textual analysis and stock returns has raised more questions that it has answered. Here is a list of nine important sets of questions that have been raised, and how our study addresses them:

First, and perhaps most importantly, how should one best measure news using word flow? One approach is to apply atheoretical methods (i.e., those with no a priori position regarding which particular words should be the focus of the analysis) to organize the flow of words in a comprehensive and unconstrained manner in order to see which parts of word flow matter for market outcomes. An alternative approach would be to identify, based on a priori criteria, key lists of words or combinations of key words to see how their presence matters for 
market outcomes (for example, Baker, Bloom and Davis 2016). A major advantage of the former approach is that it does not require researchers to know in advance what aspects of word flow are most relevant. The atheoretical approach also avoids data mining by imposing discipline on the process by which text is analyzed. There are only a few atheoretical measures of word flow to consider in empirical work: frequency, sentiment, unusualness (also known as entropy) of words, and topical categories that are identified without imposing any a priori notions of how to group words. In contrast, there is no limit to the number of a priori mappings that could be constructed based on particular words or combinations of words. A potential advantage of the alternative a priori approach, however, is that it may cut through noise by searching in more relevant places for words or phrases that have likely importance.

We adopt an atheoretical approach, and cast a broad net to see which aspects of word flow are most relevant for market outcomes. We then compare that approach with one prominent a priori approach (the Baker, Bloom and Davis measure of uncertainty), which confirms our view that an atheoretical approach may have desirable properties.

Second, which aspects of word flow should be the focus of measurement? There is a large literature showing that "sentiment" has explanatory power for returns. Articles that contain words with pre-identified positive sentiment value (as measured by a sentiment "dictionary") are associated with positive returns, while those with negative value are associated with negative returns. But sentiment is only one dimension of word flow. The frequency of the appearance of certain words or phrases (compared to their past frequency) may also be relevant, and it may also be that the context in which words appear (which we label "topics") is important to the meaning of word flow. In addition to measuring sentiment, the contextual frequency of word flow, and the way sentiment matters differently depending on context, other aspects of text flow may be 
relevant. Glasserman and Mamaysky (2016a) show that the unusualness (entropy) of word strings may have predictive power for market outcomes, especially when interacted with sentiment. As we show below, the effects of measured sentiment and frequency do vary across topic categories, so this decomposition of sentiment may be particularly useful in forecasting applications.

Our empirical approach will include these various measures of text flow and their interactions, and explore their incremental information content relative to non-textual variables often included in asset pricing studies. We also include controls that capture differences over time related to the electoral cycle, which we thought might be relevant when measuring the effects of news on market outcomes.

Third, the patterns that link frequency, topics, sentiment and entropy measures of word flow with market outcomes may vary over time. Regime changes over time ideally would be captured in a neutral manner (identifying switches endogenously using data patterns).

As a starting point, in this paper, we capture changes over time using a dividing point that is identified by principal components analysis. We show that the mapping from word flow measures to market outcomes changed somewhat after the global financial crisis. We further explore dynamic changes in coefficients using a rolling elastic net regression - which allows for model selection and coefficient shrinkage - for out-of-sample forecasting. We find that allowing coefficients to change over time is important for out-of-sample forecasting.

Fourth, given the potential importance of identifying topical context, how should one identify topics? One could adopt an a priori approach to identifying topic areas, or alternatively, employ an atheoretical approach that divides words into topic groups based on certain statistical 
properties of the corpus. Within the set of atheoretical means of identifying topics there are two common methods, namely the Louvain (Blondel et al. 2008) and Latent Dirichlet Allocation (LDA, see Blei et al. 2003) approaches, as we discuss further below. The Louvain method assigns salient words to mutually exclusive topic areas based on word co-occurrence (that is, each word belongs to only one topic area). The LDA method allows words to appear in more than one topic area (on a probabilistic basis).

After verifying with some exploratory analysis that our regression findings are similar under either approach, we focus on the Louvain method. The Louvain approach, variants of which have been applied in diverse fields from sociology (Rule, Cointet and Bearman 2015) to marketing (Netzer et al. 2012), has the major advantages of much faster computational speed, which results from the simplicity of a mutually exclusive approach to assigning words to topic areas, as well as greater ease of interpretability. ${ }^{1}$

Fifth, should all market outcomes (i.e., both changes in expected returns and changes in risk) be related to each of the identified word flow measures, or should some word flow measures be more important for risk and other measures more important for expected returns?

Our approach allows all word flow measures to matter for all of the market outcome measures. Our findings suggest that when a word flow measure has positive content for expected returns it also has negative content for changes in risk (i.e. risk, however measured, will be

\footnotetext{
${ }^{1}$ Rule, Cointet and Bearman (2015) discuss the pros and cons of various topic classification approaches, and reach a conclusion similar to ours - that co-occurrence approaches are appealing due to their simplicity and the ease of interpretability of results.
} 
lower). In other words, word flow measures largely divide into "good" and "bad" news, where bad news implies lower expected returns and higher risk, and good news implies the opposite. ${ }^{2}$

Sixth, one must decide which market measures to include in any analysis of the effects of word flow on market outcomes? Conceptually, the obvious focus, reflected in prior work, should be on expected returns and on risk. For each country, returns can be measured using the change in the value of the country stock market index. Risk, however, is a more subtle and problematic variable to measure. As is well known, if the returns process is characterized by Brownian motion and normality of the error term, then the standard deviation of returns (say, over a particular month) will be a sufficient statistic for risk. Those assumptions, however, generally are rejected, especially for emerging market (EM) countries, which exhibit pronounced momentum, and non-normality, both with respect to skew and kurtosis (see Bekaert et al. 1998, Karolyi 2015, and Ghysels et al. 2016).

Given those facts, to capture risk, in addition to using the standard deviation of returns $($ sigma $)$, we also employ the "maximum one-year drawdown." This measures, at any point in time, the maximum percentage decline that occurs from the current index value during the next year. This measure also is intended to capture the fact that "downside risk" may be treated differently from "upside risk" (the standard deviation of returns treats them as identical).

Seventh, the existing literature typically focuses on short-term analysis of individual U.S. companies or the U.S. stock market as a whole. ${ }^{3}$ Do empirical patterns that apply to individual

\footnotetext{
${ }^{2}$ In particular, there is no evidence in our data of a risk-return trade-off in predictability. Sentiment measures do not forecast higher returns because they forecast higher volatility. Instead, when they forecast higher returns, the news measures also forecast lower volatility and lower drawdowns.

${ }^{3}$ A notable exception is Froot et al. (2017), who analyze media reinforcement effects at the country index level.
} 
company stocks or the aggregate U.S. index also apply to other countries? When analyzing news and stock market behavior, should countries be lumped together or analyzed separately?

We analyze the aggregate monthly stock market returns and risks for 52 countries. ${ }^{4} \mathrm{We}$ divide countries into two groups: developed (DMs) and emerging (EMs) economies, which we believe allows for important environment differences across countries while still preserving sufficient statistical power. On the one hand, statistical power can be enhanced by a panel approach. On the other hand, important differences across countries may be lost. As we have already noted, there is a great deal of evidence suggesting that returns processes differ between EMs and DMs. Furthermore, the amount of risk and the nature of the news that drives risk differ dramatically between EMs and DMs (Beim and Calomiris 2001, Karolyi 2015). Those categorical differences likely reflect fundamental differences in political contexts, which result in different ranges of government policy choices, differences in information production for securities markets, different market liquidity (Calomiris, Love and Martinez-Peria 2012), differences in legal environment and corporate governance (La Porta et al. 1998), different fiscal, monetary and exchange rate regimes (Calvo and Reinhart 2002), differences in sovereign default risk (which is absent in most DMs but is relatively high in EMs, as described in Cruces and Trebesch 2013), and differences in the frequency and severity of banking crises (Laeven and Valencia 2014). EMs suffer larger and more frequent major drawdowns of stock returns than EMs (Kaminsky and Schmukler 2008). For all these reasons, we divide countries into EMs and DMs and perform separate panel analyses of each group of countries.

\footnotetext{
${ }^{4}$ We only use 51 countries in our panel regressions because we exclude Iceland, which experienced a drawdown of $95 \%$. Including this outlier affected coefficient magnitudes in our regression models, and therefore, we excluded it.
} 
Eighth, what source of news should one use? Given our global interest (across EMs and DMs) we need an English language news source covering many countries. Thomson Reuters generously provided their entire database of news articles from 1996 through 2015.

Specifically, our empirical study divides countries into two groups: DMs and EMs, and estimates the connections between a comprehensive set of word flow measures and market outcomes (returns, sigma and drawdown) for both samples, for the entire sample period (19982015) and for two sub-periods (April 1998 - February 2007, and March 2007-December 2015).

Finally, over what time frame should word flow predict risk and return? Much of the existing finance literature on the effects of sentiment on individual stocks' returns have focused on high-frequency predictions. Glasserman and Mamaysky (2016a) are an exception; using the U.S. stock market index, they find predictive power for risk several months following word flow. Similarly, Sinha (2016) and Heston and Sinha (2017) find that it can be useful to aggregate over longer periods of time when analyzing news for individual stocks. They find that when aggregating news over a week rather than a day one substantially lengthens the time horizon over which news forecasts returns. Weekly news predicts returns for 13 weeks, while daily news predicts returns for only two days. Motivated by these findings, we aggregate news at a monthly horizon, and then examine both one-month ahead and one year ahead predictions, and show that our country-level aggregates exhibit stronger predictive power for one year ahead returns and drawdowns than for one-month ahead forecasts of return and volatility.

Section 2 describes how we derive measures of word flow used in the study and provides a list of variables and sources for them. Section 3 presents regression results. Section 4 presents out-of-sample tests of our model. Section 5 concludes by summarizing our findings. 


\section{Data Construction, Variable Definitions, and Summary Statistics}

The analysis in this paper combines three types of data - market, macro and news - all of which are aggregated into a single data set at the month-country level. Our country level stock market index data are obtained from Bloomberg. Table 1 shows the mapping from each of our DM and EM countries to the corresponding stock market index. All index returns are converted into US dollar terms using end-of-day exchange rates from Bloomberg. For a given country, we calculate its one-month ahead return (return), its one-year ahead return $\left(\right.$ return $\left.^{12}\right)$, its realized monthly volatility (sigma) and its one-year ahead drawdown (drawdown) using these US dollar returns. Our macro data, such as interest rates, GDP growth rates, and credit ratios, are obtained from a myriad of sources, like the World Bank and the IMF, as detailed in the Appendix. Our textual data source is the Thomson Reuters Machine Readable News archive. This archive includes all Reuters News articles from 1996 to 2015, from which we use only the English language news. The measures of textual content we employ are constructed by us, as described below (also see the Appendix for more details).

Thomson Reuters News Analytics (TRNA) offers its own version of a sentiment measure as a commercial product, which has been used by Sinha (2016) and Heston and Sinha (2017), among others. The TRNA sentiment measure captures similar content to the sentiment measures we construct, but the TRNA sentiment measure is only available for a fraction of the articles in the Thomson Reuters database, and only from 2003. ${ }^{5}$ For this reason we use our own sentiment

\footnotetext{
${ }^{5}$ In response to a comment received after we completed our analysis, we purchased the TRNA sentiment data and compared regression results for the post-2003 period using our sentiment measure with those based on the TRNA sentiment measure. A detailed comparison is provided in Supplementary Appendix Tables A5-A9. We find that the two measures are correlated (correlation coefficients of the two approaches to measuring sentiment are generally greater than 0.3 and less than 0.4 , depending on topical context), and using the TRNA measure in our regression framework yields somewhat similar findings to those reported here, but the use of our measure generally results in more precise estimation and higher R-squared.
} 
measures constructed directly from the raw text of the Thomson Reuters Machine Readable News archive.

\subsection{Construction of Text Measures}

Our text processing can be broken up into four parts: (i) corpus selection and cleaning; (ii) construction of the document term matrix and topic classification; (iii) extraction of n-grams to allow for calculation of entropy; and (iv) calculation of article-level sentiment, topic and entropy measures. Here we present a high-level overview of the process. The Appendix contains more technical and methodological detail.

In the first step, we select our text corpus and then clean and preprocess it. For the EM analysis, our corpus consists of all articles tagged by Thomson Reuters with the N2:EMRG code, which indicates an article about an emerging market country. Our EM corpus consists of 5mm unique articles. Our DM corpus consists of all articles about the countries identified as developed market economies in Table 1. The DM corpus consists of $12 \mathrm{~mm}$ unique articles. All textual analysis in the paper is done separately for the EM and DM corpora.

In the second step, we calculate the document term matrix for the corpus under consideration. The document term matrix, with rows corresponding to articles and columns corresponding to words, counts the number of times a given word appears in a given article. For a given document term matrix, let us write $D_{j, w}$ for the number of times word $w$ appears in article

$j$. We restrict the words whose occurrences we count to our econ word list. This is a list of 
1,242 stemmed words, bigrams and trigrams ${ }^{6}$ that are descriptive of either market or economic phenomena (pre-stemming examples include barriers, currency, parliament, macroeconomist, and World Bank). This list was derived as follows: We began with the index from Beim and Calomiris' Emerging Financial Markets textbook. We then searched for words that co-occurred frequently in our articles database with the words in that list. The list itself, as well as the classification of each word into a topic, is available from the authors.

To define topic groups, we use the document term matrix to measure the tendency of groups of words to occur in articles together - we refer to this tendency as co-occurrence. Information about the co-occurrence of words, as measures by cosine similarity (see Appendix), is stored in the co-occurrence matrix (a symmetric matrix with a row and column for each of our econ words). The co-occurrence matrix defines a network of our 1,242 econ words, to which we apply the Louvain community detection algorithm to find non-overlapping clusters (i.e. a word can belong to only one cluster) of related words - we refer to these clusters as topics and label each one with what appears to us to be a natural topic title. Details of this procedure are given in the Appendix, but intuitively we are looking for groups of words that tend to co-occur in articles more frequently than would be expected by chance. This procedure yields five topics for each of our DM and EM corpora. ${ }^{7}$ Figures 1 and 2 show the most frequently occurring words in each of our EM and DM topics. ${ }^{8}$ For the EM corpus, we find five word groupings, which we label as: markets $(M k t)$, governments $($ Govt $)$, commodities (Comms), corporate governance and structure (Corp), and macroeconomic topics (Macro). For the DM corpus, we find similar, but not

\footnotetext{
${ }^{6}$ Bigrams and trigrams (or 3-grams) are word phrases of length two and three respectively.

${ }^{7}$ We found that recalculating topics over different subsamples of our data yielded very similar word groupings to those that were obtained over the entire sample. See the Appendix for more details.

${ }^{8}$ Figure 3 shows that the original Louvain clustering produced over 40 word groupings for each corpus, yet only 5 of these contained more than just a few words. We place words from the smaller groupings into the 5 large one for each corpus. This is discussed in greater detail in the Appendix.
} 
identical topics: markets (Mkt), governments (Govt), commodities (Comms), corporate governance and structure (Corp), and the extension of credit (Credit).

Table 2 shows that the word overlap between the topics we identify in our EM and DM corpora is often, though not always, sizeable. Our measure of word overlap is the Jaccard index, which for two sets $A$ and $B$, reports how many elements there are in their intersection divided by the number of elements in their union. The rows of the table correspond to DM topics, and the columns correspond to EM topics. For example, we see when we compare the Govt topic between our EM and DM corpora that $82 \%$ of all words common to the two topics are present in each topic separately. This indicates that the words that tend to co-occur in government-related articles in our EM and DM samples are quite similar. Similarly, the Jaccard overlap between the Mkt topic in our EM and DM samples is 59\%. There is some overlap in the Comms topic as well. We also note that there is a large overlap (of $46 \%$ ) between the Corp topic in EM and the Credit topic in DM. Our EM Macro topic has no close analogue in any of the DM topics (the closest is the DM Comms topic) - suggesting that news about EM economies tend to focus on topics of macroeconomic interest in a way that articles about DM economies do not. Perhaps this is because macroeconomic institutions in DM economies are more settled than their EM counterparts and therefore require less news coverage.

Tables 3 (for EM) and 4 (for DM) show four sample headlines of articles classified as belonging to each of the topics we identify in our analysis, which provide some examples of how our identified topics relate to articles used in our analysis. ${ }^{9}$ For example, in the emerging market corpus an article titled "Clinton says Putin can build strong, free Russia" is classified as being in

\footnotetext{
${ }^{9}$ In these tables an article is classified into topic $\tau$ if between $80 \%-90 \%$ of its econ words belong to that topic.
} 
the Govt topic. A Portuguese language article (which was wrongly coded by our data source as an English language article) entitled "Sao Paulo volta a registrar inflacao no comeco de marco" is classified - seemingly correctly - in the Macro topic. Presumably this article was included, despite the fact that it is not in English, because the relevant stemmed Portuguese words are identical to their stemmed English counterparts. While we explicitly select only English language articles from the Thomson Reuters data set, some of their language metadata is apparently incorrect. In the developed markets corpus, most of our sample articles seem to be classified correctly based on their headlines. For example "BRIEF-NQ Mobile announces termination of proposed divestment of Beijing Tianya" is in the Corp topic.

Thomson-Reuters' articles cover a wide range of topics. For example, sports articles are included, although they are often discussed from the perspectives of the economic or business implications of the sports-related event, which explains why sports articles have positive weights in the topic areas we identify. We considered restricting our sample of articles to those that were more narrowly focused on business, economics and politics topics, but we found that doing so slightly reduced the explanatory power of news for stock returns and risk, and so we retained the full sample of news articles for our analysis.

The third step of our textual analysis is the extraction of n-grams. We use n-grams, or more specifically 4-grams, to construct a measure of the entropy of a given article, following closely the methodology proposed in Glasserman and Mamaysky (2016a). An n-gram is a collection of $n$ contiguous words. ${ }^{10}$ We do not allow n-grams to cross sentence boundaries - so these are n-word phrases that appear entirely in a single sentence. Our measure of the entropy of

\footnotetext{
${ }^{10}$ The phrase "collection of $n$ contiguous" is an example of a 4-gram.
} 
a given 4-gram is the probability of observing the $4^{\text {th }}$ word in the phrase conditional on seeing the first 3 words. This conditional probability is estimated from a training corpus as follows

$$
m=\frac{\hat{c}\left(w_{1}, w_{2}, w_{3}, w_{4}\right)+1}{\hat{c}\left(w_{1}, w_{2}, w_{3}\right)+10}
$$

where $\hat{c}$ counts how frequently a given 4 -gram or 3 -gram occurred in a training corpus. Adding 1 to the numerator and 10 to the denominator is a simple way to handle cases where the 3 -word phrase that begins the 4-gram was not seen in the training corpus. In the Appendix, we discuss why this 1:10 rule is an appropriate choice.

For a given month $t$, the training corpus includes all articles from either the EM or DM corpus that appear from month $t-27$ to $t-4$ (we discuss this window choice in the Appendix). For example, consider the 4-gram "central bank cuts interest." Our conditional probability measure for this 4-gram would be high if the word "interest" very often followed the phrase "central bank cuts" in our training corpus. If many other words also followed the phrase "central bank cuts," then $m$ would be small and we would consider this 4-gram unusual. We extend the concept of entropy at the 4-gram level to the article level by calculating the negative average log probability of all 4-grams appearing in that article. For a given article $j$, this measure is given by

$$
H_{j}=-\sum_{i} p_{i} \log m_{i}
$$

where $p_{i}$ is the fraction of all 4-grams appearing in the article represented by the $i^{\text {th }} 4$-gram and $m_{i}$ is $i$ 's conditional probability from the training corpus. This measure is also known as the cross-entropy of $m$ with respect to $p$, and we will often refer to it as entropy in our analysis.

Intuitively, we characterize an article as unusual if it contains language that is unlikely to have been seen in the past. We conjecture that such new language may be needed to describe 
new market or economic phenomena, and that the presence of the latter may indicate heightened (or perhaps reduced) market risks. In the same way that the context of a news story might matter for its market relevance, the entropy of the news story may matter as well.

Finally, we combine our topic analysis with article level sentiment. Our article level sentiment measure for article $j$ is defined as

$$
s_{j}=\frac{P O S_{j}-N E G_{j}}{a_{j}}
$$

where $P O S_{j}, N E G_{j}$, and $a_{j}$ are the number of positive, negative and total words in the article. We use the Loughran-McDonald (2011) sentiment word lists to classify words as being positive or negative. This is the standard measure of sentiment that has been used in the finance literature (see, for example, Garcia 2013). Tables 3 (for EM) and 4 (for DM) show $s_{j}$ for the sample articles discussed earlier. In each topic, we report two sample articles with a very negative sentiment, as well as two sample articles with a very positive sentiment. For example, in the DM corpus the article "Euro rises above \$1.07 against dollar on war" in the $M k t$ topic plausibly receives a very negative sentiment value of -0.20 . Sometimes, the lack of semantic context causes our sentiment classification to assign an inappropriate value, given the actual meaning of the article. For example, the article “BRIEF-Moody's revises Pulte's outlook to stable from positive" which appears (appropriately) in the Credit topic is assigned a very positive sentiment score of 0.23 because it contains words like "positive" and "stable" - both positive sentiment words in Loughran-McDonald - though clearly being moved to stable outlook from a positive outlook is a mildly negative credit event. We regard these errors as inevitable noise in identifying sentiment that arises from the inherent complexity of combinations of words, and the consequent difficulty in coding sentiment of phrases using sentiment values of individual words. 
For topic $\tau$, let us define $e_{\tau, j}$ as the number of econ words in article $j$ that fall into topic $\tau$ and $e_{j}$ as the total number of econ words in article $\mathrm{j}$. Then $f_{\tau, j}=e_{\tau, j} / e_{j}$ defines the fraction of article $j$ 's econ words that fall into a specific topic (recall topics are defined as non-overlapping sets of econ words). We refer to $f_{\tau, j}$ as the frequency of topic $\tau$ in article $j$. We can decompose an article's sentiment into a context-specific sentiment measure via

$$
s_{\tau, j}=f_{\tau, j} \times s_{j}
$$

For example, an article with a sentiment measure of $-3 \%$ that was mostly about government issues with $e_{G o v t, j} / e_{j}=90 \%$ would have a government-specific sentiment of $-2.7 \%$. And its sentiment allocation to the other topics would be close to zero. Note also that since $\sum_{j} f_{\tau, j}=1$ we'll have $\sum_{\tau} s_{\tau, j}=s_{j}$, which justifies our use of the term "decomposition".

We are interested in testing in this paper whether article decomposition matters. Does negative or positive sentiment matter more or less for forecasting future market outcomes when it occurs in news stories about governments than when it occurs in news stories about macroeconomics? And how do these distinctions change when we consider DM or EM economies? Much of our empirical analysis will be focused on these and related questions.

It should also be noted that a similar decomposition can be applied to the article level entropy measure $H_{j}$. However, we did not find this decomposition to be useful and in all our empirical results report article-level entropy. Following Glasserman and Mamaysky (2016a), we compute article level context-specific sentiment interacted with entropy as follows

$$
\text { SentEnt }_{\tau, j}=f_{\tau, j} \times H_{j} \times s_{j}
$$


SentEnt is intended to capture the possibility that extreme sentiment (either positive or negative) may have more information content for future market outcomes when it occurs in articles that appear unusual (i.e. have high entropy).

\subsubsection{Aggregation of Article Data at the Daily and Monthly Level}

Once we have article level data - either entropy, context specific sentiment or entropy, or topic frequency - we aggregate these into a country level daily measure by weighting by the number of words (total, not just econ words) in the article in question divided by the total number of words in all articles about that country on a given day. For example, daily topic sentiment is

$$
s_{\tau} \equiv \sum_{j} \frac{a_{j}}{a} \times s_{\tau, j}
$$

where $a$ is the total number of words in all articles mentioning a given country on a given day. The analogous definition is applied for article entropy and frequency. The monthly measure of either sentiment, entropy, or topic frequency for a given country is the simple average of that month's daily measures.

\subsection{Data Summary and Preliminary Analysis}

Table 5 contains a brief description of the variables used in our analysis, and Table 6 contains summary statistics for those variables from 1998 to 2015. Several features of the data are worth mentioning. Compared to DM, EM returns were higher ( $1.04 \%$ vs $0.65 \%$ per month), more volatile ( $21.48 \%$ vs $19.20 \%$ annualized volatility), and subject to higher drawdowns (17.4\% vs 15.3\%). As reported by Ghysels et al. (2016), EM returns are also more right skewed as retpl 
(the positive portion of returns) averages $3.9 \%$ for EM and only $2.8 \%$ for DM, and retpl is also more persistent for emerging markets with an AR(1) coefficient of 0.12 vs 0.05 for developed markets. Emerging markets grew faster ( $g d p$ ), had higher inflation (gdpdefaltor), higher nominal interest rates (rate), and lower private sector debt to GDP ratios $(c p)$. The average number of articles per day for EM countries is 26.0 and for DM countries is 106.7. The fraction of these articles dealing with Corp, Govt, and Mkt topics are similar, and EM countries have many more Comms articles (15.9\% for EM vs only $2.7 \%$ for DM). Finally, the average article level entropy for both corpora is roughly 2.45 .

\subsubsection{Structural Break Around the Financial Crisis}

Figures 4 and 5 provide factor loadings and plots for each topic category related to the first two principal components for the $140 \mathrm{EM}$ ( 5 series for 28 countries) and $120 \mathrm{DM}$ ( 5 series for 24 countries) time series of country-month-topic sentiment. The first principal component (both for EMs and DMs) tracks the aggregate time series of market movements. For both EMs and DMs, the second principal component appears as a step function with a break at the timing of the global financial crisis. Interestingly, the second principal component has different factor loadings (both in sign and in absolute value) across different topic areas. Govt sentiment enters negatively and $M k t$ sentiment enters positively for the second principal component. That means that, prior to 2007, the sentiment score of market topic-related articles was more positive than government topic-related articles. That higher relative magnitude reversed after 2007, and the

sentiment score of market topic-related articles became relatively negative in comparison with government topic-related articles. In our regression findings below, we find important related 
breaks in regression coefficients (and some reversals in sign) that are related to this structural break in 2007-2008.

\section{$\underline{\text { 3. Empirical Findings }}$}

Here we present our empirical findings about the connections between various measures of word flow and our measures of expected return, the standard deviation of returns (sigma) and cumulative downside risk (drawdown). As a starting point for our analysis, following Tetlock et al.'s (20008) and Hendershott et al.'s (2015) analysis of company returns, we perform an event study of country stock returns around days in which sentiment scores for news for a given country are extremely positive or extremely negative. Specifically, we identify days for which positive or negative sentiment lies in the top decile of the historical distribution, and we do this for each of the five topical categories, separately for EMs and DMs. Figures 6 and 7 plot cumulative abnormal returns (for EMs and DMs, respectively) for the 10 days prior to and subsequent to the identified event dates (which appear as day 0 in the figures). Abnormal returns for each country are the residuals from regressing that country's US dollar returns on a constant and the appropriate MSCI index (either DM or EM) over the entire sample period. ${ }^{11}$ We plot these abnormal returns separately for positive and negative news dates, along with standard error

\footnotetext{
${ }^{11}$ When running lagged regressions prior to the event date as the control, we noticed that the pre-event estimated alpha was correlated with the news event itself. Positive (negative) news days tended to be preceded by positive (negative) alphas. Because of this, the pre-event window was not an appropriate baseline return model, and therefore we used a regression over the entire sample as the control.
} 
bands. ${ }^{12}$ We also plot (in between the positive and negative top deciles) the results for the middle decile $\left(45^{\text {th }}-55^{\text {th }}\right.$ percentile $)$ as a placebo. ${ }^{13}$

Interestingly, the plots for EMs and DMs are quite similar for the four common topical categories (Mkt, Govt, Comms, and Corp), and surprisingly, are also quite similar for the fifth (dissimilar) topical category (Macro for EMs, and Credit for DMs). For both sets of countries, the shapes of cumulative abnormal returns around event dates are often similar for negative and positive news, although there are also some interesting asymmetries. Positive and negative cumulative returns tend to occur in advance of, respectively, positive and negative big news days, with the exception of negative news days for Govt and Comms in DMs and also positive news days for Comms in DMs.

One noteworthy aspect of the event studies is that news events appear to cause more of a market reaction in our DM sample than in our EM sample (note the bigger event day price jump in the former compared to the latter). This reflects either more timely reporting by Reuters in their developed market news bureaus, or information leakage (perhaps due to weaker regulatory enforcement) in EM economies.

It is interesting to compare our event studies to those in Tetlock et al. (2008) - their Figure 3. Our country level abnormal returns, relative to their US firm level abnormal returns, have more pronounced pre- and post-event drifts around negative news events - a finding that

\footnotetext{
${ }^{12}$ Our standard errors are calculated under the assumption of serial and cross-sectional independence of events. Both assumptions are problematic in our data. Furthermore, it is possible that the pre-event country index performance has a causal relationship to the news event itself. Proper inference in this setting is beyond the scope of the present paper, and our standard errors should be interpreted with this caution in mind.

${ }^{13}$ The decile cutoffs are calculated over the entire sample. Note that the numbers of events in our three decile buckets are not the same. We bucket by the daily sentiment in each of the topic categories. Some of these event dates are either on non-trading days (e.g. weekends) or within 10 days of the start or end of the sample. We do not include such event days for the calculation of abnormal returns.
} 
seems to hold for both EM (for Mkt and Comms topics) and DM (for Mkt and Credit topics) markets. In Tetlock et al. (2008) abnormal returns on stocks seem to be very weakly meanreverting following negative news. Both their results and ours - in some cases - show a weak positive drift after positive news events. This more pronounced post-event country-level drift after negative news suggests that long/short news-based strategies may be more profitable at the country level than the individual stock level. Though tentative, this is potentially further evidence of the relative micro efficiency and macro inefficiency of markets (see Glasserman and Mamaysky $2016 b$ for a theoretical exploration of this question).

In results not reported here, we investigated whether these extreme positive and negative news days are predictable based on prior days' sentiment scores. We found no evidence of a preevent drift in sentiment - sentiment did not decrease (increase) in the 10 days leading up to a bottom (top) decile negative sentiment day. Our evidence suggests that news reports respond more slowly to underlying market or economic developments than do returns. This does not imply, however, that word flow measures lack predictive content for returns. Indeed, as our monthly analysis below shows, lagged word flow measures (including sentiment) do have predictive content for return, sigma and drawdown.

\subsection{Panel Regression Analysis of Risk and Return in EMs and DMs}

Turning to our regression analysis, in Tables 7-12, we report regressions employing country-month observations, divided into EM and DM samples, for our three dependent variables (return ${ }^{12}$, sigma and drawdown). ${ }^{14}$ We regress month $t$ values of the dependent

\footnotetext{
${ }^{14}$ Regression results for one-month ahead returns are shown in Supplementary Appendix Tables A3 and A4.
} 
variables on lagged (either $t-1$ or $t-2$ ) values of our explanatory variables. Our regressions are panels with country-month data, and country fixed effects. Section A.6 in the Appendix discusses some associated econometric issues. In each table, we report nine different regressions, which consist of three regressions for each of three time periods. The three time periods are April 1998-December 2015 (the entire sample period), April 1998-February 2007 (the pre-global financial crisis period), and March 2007-December 2015 (the post-global financial crisis period).

Within each time period we first report a Baseline regression, which includes control variables (non-textual predictors of the three dependent variables). Controls include two lags of monthly returns (for the sigma regressions we use retmi $=\max (-$ return, 0$)$ instead based on the findings in Bekaert and Hoerova 2014), two lags of monthly volatility, and single lags of other financial, macroeconomic, and electoral cycle control variables, all of which are described in Table 5. We included indicator variables that capture electoral timing by dividing time periods into pre-election and post-election periods, as described in Section 2 and the Appendix. ${ }^{15}$

In addition to the Baseline regression, for each time period, we report two additional regressions which examine the incremental predictive power of various word flow measures. Each of these specifications includes country level monthly entropy (entropyt-1), the monthly average of daily article counts (artcount $t_{-1}$ ), and the monthly frequency measure $f_{\tau}$ for each topic. The first specification (in column labeled Sent) includes each topic sentiment measure in its

\footnotetext{
${ }^{15}$ There is a large literature on forecasting country-level returns. The general conclusion has been that stock-level effects largely are also present at the country index level. For example, lagged valuation ratios and lagged interest rates have all been shown to forecast country-level returns (see Asness et al. 1997, Ang and Bekaert 2007, Angelidis and Tessaromatis 2017, Hjalmarsson 2010, among many others). Also, momentum and reversal effects have been documented (Asness et al. 2013 and Richards 1997). We control for these effects, and also introduce other market (exchange rate changes) and macroeconomic (inflation, GDP growth, etc.) variables as additional controls.
} 
simple form, i.e. $s_{\tau, j}$. The second specification (labeled SentEnt) includes the entropy interacted versions of the sentiment variables (SentEnt $\tau_{\tau, j}$ from (5)) . In the tables, we label rows showing the loadings on $s_{\tau, j}$ and SentEnt $\tau_{\tau, j}$ as $s M k t, s G o v t$, and so on; the column heading specifies whether these refer to the simple or the entropy interacted topic sentiment. All sentiment measures, except entropy, are normalized to have unit variance at the country level. See the Appendix for more details about our regression specifications.

Our findings with respect to Baseline variables' effects generally are consistent with prior studies and will not be commented on further here. ${ }^{16}$ Our results differ across EMs and DMs (coefficient values are not consistent across the two groups of countries), and overall, return, sigma and drawdown tend to be more predictable for DMs (as measured by higher R-squared). This confirms the view that the nature of news, and the range of potential news outcomes, differ in EMs and DMs (reflecting important differences in the political and economic environments, which are reflected in returns outcomes). Further observations follow.

Similarity of effect for returns and risk. When a word flow measure has a positive (negative) effect on return, it often tends to have a negative (positive) effect on sigma and a negative effect on drawdown. In other words, news contained in word flow is often either "good" or "bad" for all three dependent variables, where good news increases return and reduces risk measured either by sigma or drawdown. In fact, we never observe a coefficient in a return regression that is of

\footnotetext{
${ }^{16}$ We observe, as have others (e.g. Fama and French 1988) very little forecasting power for one-month ahead returns. One interesting finding is that GDP growth is negatively associated with returns and positively associated with drawdown. We can think of many potential explanations for this finding. First, it may be that positive GDP growth raises the probability of contractionary monetary policy, which may be bad news for the stock market. Second, it is possible that GDP growth serves as a proxy for states of the world in which coefficients on other variables in the model (such as momentum or value) would change if the model permitted time-varying parameters.
} 
the same sign (and statistically significant) as the same variable's coefficient in a sigma or drawdown regression.

Incremental R-squared. The economic importance of word flow measures (incremental contribution to R-squared) tends to be relatively small for return and sigma, both in EMs and DMs, compared to their contribution to return ${ }^{12}$ and drawdown. Volatility (sigma) is the most predictable of the three dependent variables, with values ranging from 0.45 to 0.48 in DMs and from 0.32 to 0.40 for EMs. The usefulness of Baseline control variables is especially high for predicting sigma in DMs, and the incremental contribution of word flow to sigma is small in DMs.

Effects of Specific Text Measures. In EMs, the economic importance of word flow measures is higher for all three measures, but it is especially high for return ${ }^{12}$ and drawdown. In DMs, Rsquared increases for return ${ }^{12}$ and drawdown, respectively, too (rising from 0.16 to 0.21 , and from 0.26 to 0.32 for the sample period as a whole). In EMs, the absolute value of the increase is slightly larger, but the increase in R-squared as a proportion of Baseline R-squared is much larger: for the sample period as a whole, including text measures roughly doubled the R-squared for both return ${ }^{12}$ and drawdown (from 0.07 to 0.13 and from 0.06 to 0.12 ). For the pre-crisis period, that difference between EMs and DMs is even greater: for EMs, R-squared for return ${ }^{12}$ and drawdown rise from 0.02 to 0.13 and from 0.08 to 0.22 , while for DMs these increase from 0.27 to 0.30 and from 0.40 to 0.45 . We interpret this as confirming that the nature of news tends to be different in EMs and DMs: In EMs, where events reported in the news often contain information about fundamental shifts in political and economic regimes (which is relatively absent in DMs) the incremental value of tracking word flow is greater. 
The impacts of individual text flow measures on annual returns and drawdowns often are economically large. In DMs, text measures are only significant for one year ahead returns (as shown in Table 7) during the period after 2007. During that period, a one standard deviation increase in entropy is associated with a $3.7 \%$ higher return over the next year (the product of its standard deviation, 0.17, and its coefficient, 21.87). A standard deviation increase in sMkt is associated with a $5.2 \%$ increase in return, while a standard deviation increase in $s G o v t$ is associated with a $3.9 \%$ reduction in return.

Magnitudes for drawdowns (shown in Table 11) are comparable for the aforementioned variables (and signs are opposite), with the exception of the drawdown consequences of an increase in entropy, which are about half as large in absolute value. Additionally, in the drawdown regressions for the earlier subperiod, entropy and sCorp are statistically significant. A one standard deviation increase in entropy now forecasts an increase in drawdown (of roughly the same absolute value, and the opposite sign as observed for the later period). A one standard deviation increase in $s$ Corp forecasts a $1.5 \%$ decrease in drawdown.

In EMs, as shown in Table 8, more text flow measures are statistically significant for one year ahead returns. A standard deviation increase in artcount forecasts a $10.5 \%$ decline in returns in the early subperiod; there is no significant effect in the later subperiod. entropy does not enter significantly in either subperiod. $f M k t$ switches signs from a large negative returns effect $(-11.0 \%$ per standard deviation) in the earlier period to a large positive effect $(8.8 \%)$ in the later period. $f$ Govt enters negatively in the earlier subperiod with a large magnitude (-10.4\%), but it does not enter in the later period. $s$ Corp enters negatively in the later period $(-8.2 \%)$, but not in the earlier period. fCorp enters negatively in the earlier period, but not in the later period. fMacro does not 
enter significantly in either subperiod, but its sign is consistently positive, and for the combined period, it shows a large and statistically significant effect of 5.9\%.

More variables are statistically significant in the EM drawdown regressions (Table 12), often in both subperiods. Coefficient magnitudes are similarly large and, when statistically significant, are of opposite sign to those observed in the returns regressions. A one standard deviation increase in entropy flips from forecasting an increase in drawdowns of 5.9\% $(0.17 \mathrm{x}$ 34.498) in the earlier subperiod to forecasting a decrease of 3.8\% in the later subperiod. sComms enters negatively in drawdowns, which mainly reflects its forecasting power pre-2007.

Entropy Interactions. Somewhat contrary to the findings in Glasserman and Mamaysky (2016a) (which focused on financial corporations' and US stock market returns, rather than country returns around the world) we do not find that interacting sentiment measures with entropy, the SentEnt specification, adds much explanatory power. Coefficient magnitudes and Rsquareds sometimes rise and sometimes fall across Sent and SentEnt specifications, but the changes tend to be small; interacting sentiment with entropy adds little. By itself, however, entropy enters as a significant in-sample predictor of drawdown for DMs and EMs in both subperiods, of sigma in EMs for the pre-2007 sub-period, of return in DMs in the post-2007 subperiod and of EMs in both sub-periods, and of return ${ }^{12}$ for DMs in the post-2007 period. ${ }^{17}$ Time Variation in Coefficients. Consistent with our principal component discussion in Section 2, we find important differences in coefficient values for word flow measures over time - that is,

\footnotetext{
${ }^{17}$ As we discuss below, out-of-sample results shown in Figures 10-12 and Appendix Figure A2 confirm the importance of including entropy in the model. We find that entropy is chosen for inclusion in the parsimonious elastic net model, for both EMs and DMs, for return and return ${ }^{12}$, for drawdown, and for sigma, although its importance and its sign vary over time. See also the discussion in Section 3.2.
} 
differences between the pre-2007 and post- 2007 periods. Tables A1 and A2 in the Supplementary Appendix summarize our panel results by subperiod. A "+" ("-") in the table indicates that an explanatory variable enters with a positive (negative) coefficient, and is significant at the $10 \%$ level or better. The symbol " $\varnothing$ " indicates that the explanatory variable is not present in that specification (for example, return $t_{t-1}$ is not present in the sigma panels). For DMs, negative coefficients on return for fGovt and sGovt are a feature of the post-2007 subperiod, as is the positive coefficient for return for $s M k t$ and $s$ Corp. For EMs, positive fGovt is associated with larger drawdown in the earlier sub-period, but not in the later. For EMs, the coefficient on entropy in the return ${ }^{12}$ regression is zero across the two sub-periods, while the coefficients on entropy in the drawdown regressions flip from positive to negative. For DM return $^{12}$, entropy matters (positively) only in the post-2007 period. entropy has no effect on DM sigma. For DM drawdown, entropy flips from positive significant to negative significant as we move from the earlier to the later sub-period. This sign flipping for entropy is examined in more detail in Section 3.2 below.

Sign of Sentiment and Market Outcomes. Topical context matters for the influence of frequency and sentiment. Coefficients for sentiment or frequency can be positive or negative, depending on the topic area, and depending on the period. There is no general finding that positive sentiment is always associated with good news. In DMs and EMs, positive sGovt or fGovt can be bad news, and positive $s$ Corp or fCorp can also be a negative news event; whereas positive sMkt is typically good news for DMs and positive sComms and fMacro are typically good news for EMs. Clearly, there is something to be gained by considering the context in which positive or negative sentiment is expressed. Note that sentiment is statistically significant as bad news only in the later sub-period (although frequency of market, government and corporate news 
is negative in EMs in the earlier subperiod). sCorp has a significant negative sign for EM return and return $^{12}$ and marginally negative for DM return ${ }^{12}$ and a significant positive sign for drawdown in EMs only for the later sub-period; and sGovt has a significant negative sign for DM return and marginally negative for return ${ }^{12}$ and a significant positive sign for drawdown only for the later sub-period.

One interpretation of our findings on sentiment is that negative sentiment can indicate good news if the negative sentiment is describing problems that government actions are trying to address. The notion that negative sentiment in the context of government responses is reflecting positive policy news events could also explain the post-crisis timing of the surprising coefficients for sentiment. In Section 3.4, we show that Govt and Corp sentiment and frequency both predict increases in future economic policy uncertainty (Baker, Bloom and Davis 2016) for DMs, which suggests that a policy channel is potentially at work. A similar pre- and post-crisis difference in influence could explain the observed sign flipping with respect to entropy. In the pre-crisis period, unusual word flow generally indicates risky times, but in the context of the post-crisis period, unusual word flow may be associated with unprecedented policy actions.

\subsection{Pre- and Post-Crisis Differences in the Meaning of News Flow}

To address this pre- and post-crisis interpretation of the two anomalies observed above - the negative news content of $s$ Corp and $s$ Govt in the post-2007 sub-period, and the flipping of the sign on entropy to imply positive news content in the post-2007 sub-period - we take a closer look at the changing patterns of co-occurrence among entropy, sentiment, and topical frequency over time. To examine the nature of the role of crisis influences, we divide the post-February 
2007 time-period into two sub-periods: the global crisis period from March 2007 to August 2011 (the midpoint of the post-February 2007 period), and the sub-period after August 2011. By splitting the post-February 2007 period in half, we are able to investigate whether post-crisis differences reflect changes that persist throughout the period, or changes that are only related to the onset of the global financial crisis. As before, we consider EM and DM countries separately.

Figures 8 and 9 display our results, for EM and DM countries respectively. We find that there are, indeed, changes in the patterns of co-occurrence among entropy, sentiment and topical frequency across time, and that these differ in interesting ways for EMs and DMs. In each Figure, we plot sentiment and frequency by topic first for all country-days within each subperiod, and additionally, for country-day observations in the top fifth percentile of entropy. Each chart shows the difference between the average country-day sentiment in that sub-period/entropy grouping (e.g., the early subsample-high entropy group, or the late subsample-average entropy group) and the full sample average, normalized by the full sample standard deviation. For example, the top-left chart in Figure 9 shows that in the 1996-2007 time period for DMs, average government sentiment was 0.15 standard deviations lower than the full sample average, whereas credit sentiment was 0.05 standard deviations higher. Our focus is on how sentiment and frequency by topic vary across time and across high versus typical entropy days. Our interpretation is that high entropy days contain particularly informative news flow, and are therefore worth singling out for analysis.

With respect to the top two panels of Figures 8 and 9, using all the articles in each subperiod, we observe substantial changes over time in topical frequency and topic-specific sentiment, which differ between EMs and DMs. This variation could account for the fact that our regression specifications in Tables 7-12 gained little from including interactions between entropy 
and sentiment (contrary to Glasserman and Mamaysky 2016). It may be that modeling sentiment as topic-specific and including topical frequency as a regressor, in an environment with such dramatic change over sub-periods in topical frequencies, captures much of the interaction between entropy and sentiment that would not otherwise be captured.

Conditional on observing high entropy country-days in EMs, the relative frequencies of the five topics are nearly constant over time. High entropy days in EM are associated with fewer market and more government related articles. Interestingly, high entropy days in EM are associated with generally lower sentiment levels across all topics except markets relative to average entropy days. Furthermore, high entropy EM days exhibit important changes over time in topic-specific sentiment. In high entropy days, government topic-related sentiment becomes less negative during the March 2007-August 2011 sub-period than it was before, commoditiesrelated sentiment scores become much more negative, and other topics show little change. In other words, unusual news related to commodities during the height of the global crisis tended to be negative in EMs. News related to government had slightly less negative sentiment during high entropy days that it had in the first period. EM country discussions related to government (which always tend to be sentiment negative in high entropy days) are less sentiment negative during the height of the global crisis. After August 2011, the topic-specific sentiment scores for high entropy days in EMs revert to their pre-March 2007 pattern.

As in EMs, high-entropy days in DMs are typically associated with lower sentiment in all topic areas (except markets). The sub-periods patterns for DMs during high entropy days are somewhat different however. First, for the pre-March 2007 sub-period, the high-entropy-day topical sentiment scores are quite similar to those of EMs. Second, as in EMs, during the second sub-period government-related articles on high entropy days are less negative than before, 
although they are still very negative relative to average entropy days in that sub-period. But for DMs, all the other topical areas on high-entropy days become more negative in their sentiment scores during the post-February 2007 period (with commodities-related sentiment scores showing the least change). It is not surprising that unusual DM news days related to commodities during the crisis were less negative than for EMs, given that DMs tend to be users rather than producers of commodities relative to EMs. Neither is it surprising that DMs, where the global crisis originated (with housing and banking crises originating in the U.S., Ireland, Spain, and the U.K.), are the countries where unusual news during the post-February 2007 period became particularly negative for market, corporate, and credit topics.

Even more striking is the fact that DM sentiment patterns for high entropy days did not revert to the pre-March 2007 patterns, as they did in EMs. Instead, DMs saw a continuation of the post-February 2007 topic-specific patterns for sentiment scores. It appears that the changes in the structure and content of news related to the onset of the crisis were more persistent in DMs, where the crisis and policy reactions to it were more long lasting. In additional tests not reported here, we investigated whether that persistence of DM sentiment negativity for the four nongovernment topic areas (relative to the first sub-period) is driven by a sub-sample of Eurozone or European countries. We found that it was not isolated to Europe or the Eurozone, but reflected persistent changes associated with the crisis that applied to DMs more generally.

The patterns observed in Figures 8 and 9 reinforce the interpretation that the two anomalies reported in Tables 7-12 - the negative news content of $s$ Corp and $s$ Govt in the post2007 sub-period, and the flipping of the sign on entropy to imply positive news content in the post-2007 sub-period - are related to how news coverage and its meaning change during a crisis. 


\subsection{Persistence of Effects and Endogeneity of News: A Panel VAR Approach}

It is noteworthy that the measured effects of news are greatest at long (one-year) time horizons.

This implies that our news measures likely capture fundamental economic influences rather than transitory "animal spirits" (see also Sinha 2016). ${ }^{18}$ In the Supplementary Appendix, we provide another perspective on the duration of news relevance by constructing Panel Vector Autoregressive (Panel VAR) models, separately for DMs and EMs, which measure the linkages among sentiment, entropy, monthly return and monthly volatility. ${ }^{19}$ These results are reported in Figures A3-A6. This approach is also useful for gauging the extent to which news may itself reflect past market outcomes. Because of the need to constrain the dimension of the model, we collapse the various topical sentiment measures into a single sentiment index, which - by ignoring the topical context - understandably reduces the measured importance of sentiment, compared to the results reported above. We report two versions of the model: one that puts the news variables first in the ordering (sentiment, followed by entropy), the other that puts the return and volatility measures first, followed by sentiment and then by entropy.

We find that, with minor exceptions, the effects of sentiment and entropy on returns are similar for EMs and DMs. When sentiment and entropy are first in the ordering, they both produce positive return responses in the first two months after the shock with no evidence of subsequent mean-reversion - suggesting both are capturing long-term news and not transitory

\footnotetext{
${ }^{18}$ Shiller (2017) argues that "animal spirits" can, in fact, have large fundamental economic effects. In the present work, we are not able to distinguish effects of long-lived animal spirits from news that forecasts economic fundamentals, but we are able to reject the view that the news that drives market changes reflects short-lived animal spirits. Shapiro et al. (2018) show that text-based sentiment measures forecast future macroeconomic outcomes in the US; Thorsrud (2016) presents similar evidence for Norway.

${ }^{19}$ We estimate the VAR using monthly data with two lags and country fixed effects. The variables in the VAR have units given in Table 6, except the sentiment measure, which scales to have unit variance. We are constrained to include only variables that capture monthly variation. In particular, we do not include twelve month returns or drawdowns in the VAR models. However, our impulse response functions allow us to gauge the persistence of shocks to returns and volatility over many months.
} 
"animal spirits." When sentiment and entropy are second in the ordering, there is less evidence of persistent effects on returns, but this is because we do not differentiate sentiment according to its topical context; it is likely that this approach aggregates positive and negative responses across different topics. ${ }^{20}$ Similarly, for both EMs and DMs, positive sentiment forecasts drops in realized volatility that persist for over a year after the initial shock. This is true regardless of the ordering of the VAR. In the case of DMs, entropy shocks depress realized volatility for several months when entropy is the second variable in the system, and have no effect when entropy is last in the system. Interestingly, in the case of EMs, entropy shocks increase realized volatility regardless of the ordering of the variables. Sentiment and entropy are also dynamically related: shocks to either of these variables produces protracted negative results in the other.

We also find that intertemporal influence flows in both directions. Shocks to returns and volatility have significant, and sometimes protracted influences on sentiment and entropy. Return shocks increase sentiment and decrease entropy, while realized volatility shocks decrease sentiment and increase entropy, again regardless of the ordering. These results highlight the importance of examining long-term cumulative effects of news on returns and drawdowns, and of including lagged measures of returns and volatility, as we do in our above models that evaluate the predictive importance of news flow for future returns, volatility, and drawdowns. ${ }^{21}$

\footnotetext{
${ }^{20}$ Our panel regressions in Table 7-12 and our out-of-sample results in Section 4 both show that topic sentiment is important for future market outcomes even after controlling for lagged returns and volatility. With only 18 years of monthly data, we do not believe we can reliably estimate a VAR with topic-specific sentiment (with 2 lags this requires estimating two $8 \times 8$ coefficient matrixes).

${ }^{21}$ Our modeling of the effects of text on returns, volatility and drawdown in Tables 7-12 employs one lag of each of the twelve text measures, but two lags of returns and of volatility. In results not reported here, we also experimented with adding additional lags of text measures. Doing so slightly improves the statistical significance of text measures in some cases, raises R-squared slightly, and sometimes diminishes the importance of non-text measures. Overall, the effect of adding additional lags of text measures is small, and usually divides the explanatory power captured in the one-lag specifications across the greater number of lags of the text measures in the expanded version. We report only the one-lag specifications of text measures because doing so is more conservative, and avoids falsely attributing effects to text measures that can be explained by lagged volatility or returns.
} 


\subsection{Comparison with Baker, Bloom and Davis' A Priori Approach}

The Baker, Bloom, Davis (BBD) (2016) index of economic policy uncertainty (EPU) measures the frequency with which newspapers in a given country mention the words "economy" and "uncertainty", along with references to political acts or actors in the same article. For a subsample of EM and DM countries, it is possible to compare our approach to measuring news with that of BBD (2016). Those countries include 11 DMs (the U.S., Canada, Germany, the U.K., Italy, France, Spain, Netherlands, Japan, Australia and Ireland), and 6 EMs (Chile, China, South Korea, Brazil, Russia and India). Although these DM and EM sub-samples are a small proportion of the total number of countries in our sample, they represent a very large proportion of the total economic activity in the larger sample, and therefore, this is a highly relevant subsample. Our sample time frame is from 1998 to 2015.

In Table 13 we show that our word measures can explain substantial future variation in the BBD uncertainty measure (Table 13 shows a panel regression of the time $t$ value of $E P U$ on time $t$-1values of macro control variables and our text measures). It is interesting to note that we explain a much larger portion of future EPU variation in the DM sample than the EM sample. High Mkt sentiment and frequency, high Macro frequency for EM and positive Credit sentiment for DM forecast lower future EPUs; whereas high Govt and Corp sentiment and frequency in DM, as well as higher article counts in EM, forecast higher future EPUs. Perhaps this is one channel through which positive sentiment and high frequency in the Govt and Corp topics forecast adverse market outcomes, as we discussed in Section 3.1.

Of greater interest is the explanatory power of the BBD economic policy uncertainty measure $\left(E P U_{t-1}\right)$ for return ${ }^{12}$, sigma and drawdown, both by itself and in regressions that 
include our word flow measures. ${ }^{22}$ Tables 14 and 15 evaluate the incremental explanatory power of the BBD measure for those three variables. For each variable we report four regressions: a Baseline regression that includes neither our word flow measures nor the BBD measure, a second regression that includes only the BBD measure, a third that includes only our word flow measures, and a fourth that includes both the BBD measure and our word flow measures.

For DMs, in the second regressions for each variable in Table 14, the BBD measure does exhibit incremental explanatory power, but the effects are small. R-squared for return ${ }^{12}$ is increased by 0.008 , for sigma by 0.002 , and for drawdown by 0.011 . In contrast, including our word flow measures raise R-squared by much larger amounts. Furthermore, as shown in the fourth regressions for each dependent variable, in the presence of our measures, the BBD measure loses its statistical significance. In other words, the part of the BBD measure that contains incremental explanatory power for return $^{12}$, sigma and drawdown is subsumed by our word flow measures, and our word flow measures also contain additional explanatory power.

For EMs, the second regression results shown in Table 15, for return ${ }^{12}$, sigma and drawdown, show that the BBD measure adds almost no incremental explanatory power for all three variables relative to the Baseline. Only in the return ${ }^{12}$ regression does the BBD measure enter significantly, and then only in the specification that includes all our text measures (adding the BBD measure there results in a low R-squared increase of 0.008). In contrast, for EMs, adding our word flow measures meaningfully increases R-squared for all three specifications. We conclude that our atheoretical approach provides a more effective means of distilling the information contained in news stories that is relevant for market return and risk.

\footnotetext{
${ }^{22}$ It should be noted that Baker, Bloom and Davis (2106) argue that economic policy uncertainty is useful in forecasting macroeconomic - not market - outcomes.
} 


\section{Out-of-sample tests}

There are two important reasons to explore out-of-sample forecasting properties of our model. First, as the above discussion of changes in estimated coefficients reveals, we observe substantial variation over time in coefficient estimates reported in Tables 7-12, where the timing of the split was suggested by the principle components analysis reported in Figures 4-5. Given that variation, it is unclear whether a forward-looking application of our model would produce useful forecasts of market return and risk. Second, the baseline and augmented models from Section 3 contain many explanatory - text and non-text - variables which make them susceptible to overfitting in any given sample.

When over-fitting is a concern, the typical solution is to penalize coefficient estimates by shrinking their absolute value based on an objective function that weighs each (normalized) coefficient's contribution to explanatory power (which receives a positive weight) against the magnitude of that coefficient (which receives a negative weight). We use the elastic net estimator (implemented in the glmnet package of Hastie and Qian 2016), which combines the least absolute shrinkage and selection operator (lasso) regression, introduced by Tibshirani (1996), with a ridge regression, to ameliorate this over-fitting problem. In our panel setting, we estimate rolling five-year regressions using the elastic net objective function, which is given by

$$
\min _{\beta} \frac{1}{2 N} \sum_{i, t}\left(y_{i, t}-x_{i, t-1}^{\prime} \beta\right)^{2}+\lambda\left(\alpha\|\beta\|_{1}+(1-\alpha)\|\beta\|_{2}^{2} / 2\right)
$$

where $N$ is the total number of observations in the regression, $y_{i, t}$ is the response variable, $x_{i, t-1}$ is a vector of the predictors, $\|\beta\|_{1}$ is the L1-norm of the coefficients (the sum of the absolute values of the $\beta$ vector) and $\|\beta\|_{2}^{2}$ is the L2-norm squared (the sum of the squares of the 
$\beta$ coefficients). ${ }^{23}$ In this specification, we implement country fixed effects by constructing demeaned $y$ 's and $x$ 's within each country grouping - so a constant in the above regression is not necessary. The choice of $\lambda$ determines the penalty applied to the blended L1- and L2-norms of the coefficients. This parameter is selected in each 60 -month window to minimize the crossvalidation error. We set $\alpha=0.75$ though this choice has little effect on the predictions obtained from the model (setting $\alpha<1$ improves the numerical behavior of the algorithm, as discussed in Hastie and Qian 2016).

Even a powerful model selection procedure has a hard time when confronted with too many explanatory variables and a relatively small data set. Therefore, we impose some structure on our estimation by using only a subset of our non-text variables for the out-of-sample tests: one-month returns (for our volatility model we use only the negative portion of returns), onemonth realized volatility, our value measure, the private sector credit-to-GDP ratio, and the local interest rate. Except the credit-to-GDP measure, all of these proxy for well-known asset pricing effects. The credit-to-GDP ratio was very important in the in-sample regressions (perhaps because of its predictive power for returns around the financial crisis), and so we keep it for the out-of-sample tests. These five variables, with a country fixed effect, constitute our out-ofsample Baseline model. Since we selected variables with known forecasting power for the Baseline model, we effectively have raised the bar for our text measures to add any value. ${ }^{24} \mathrm{We}$ keep all our text measures for the out-of-sample tests, except we drop commodity frequency and sentiment because these were unimportant in most of our full sample regressions. By dropping

\footnotetext{
${ }^{23}$ One important subtlety in the out-of-sample estimation for 12-month ahead returns and drawdowns is to truncate the measured 12-month ahead outcomes in the pre time- $t+1$ estimation window to ensure that they do not overlap with the $t+1$ through $t+12$ outcome that we are trying to forecast out-of-sample.

${ }^{24} \mathrm{Had}$ we selected more non-text variables, the out-of-sample performance of the Baseline model would be degraded because the elastic net would have too many degrees of freedom. Choosing variables that we know will work a priori makes the Baseline model a tougher comparison.
} 
only two text measures, as opposed to many non-text measures, we believe we are being conservative in our out-of-sample tests.

An elastic net regression performs both model selection - many of the $\beta$ 's can be set to zero - and shrinkage - the non-zero coefficient estimates are smaller than their OLS counterparts. A measure of the degree to which elastic net coefficients are smaller than their OLS counterparts is the ratio $\|\beta\|_{1} /\left\|\beta^{O L S}\right\|_{1}$. In our empirical results this ratio (reported in the upper left-hand corners of Figures 10-12 and A2) ranges from close to zero, to nearly 100 percent, meaning that the elastic net sometimes chooses an optimal in-sample model with no explanatory variables (often this happens for our 1 month return forecasting regressions), and sometimes chooses a model with coefficient estimates almost as large as their OLS counterparts (for example, in many windows for forecasting 12 month ahead returns).

Figures 10-12 show significant changes over time in the elastic net coefficient estimates for the variables in our model, including the text measures. Coefficients magnitudes, when nonzero, are large and similar to the statistically significant coefficients identified in Tables 7-12, and have similar temporal patterns. For example, the flip in the sign of entropy for EM and DM drawdowns and 12-month ahead returns is visible in the elastic net estimates. When interpreting coefficient magnitudes it is important to bear in mind that multicollinearity (which, by construction, is not apparent in elastic net estimates) leads to some non-comparability of coefficient magnitudes reported in Tables 7-12 and in Figures 10-12. Nevertheless, the elastic net results reinforce the message of Tables 7-12 about coefficient magnitudes and their variation over time. One year-ahead return and drawdown display similar pictures (with opposite signs) for individual variables for EMs and DMs. For example, both sets of results show similar changes in model fit over time. Value plays an important but varying role in the regressions for 
both EMs and DMs, as do $s$ Govt and sMkt. Rate is important, but varying, in DMs. artcount and fMacro are important, but varying, in EMs. Note that in out-of-sample tests, as well as in our panel analysis, positive $s M k t$ tends to be good news for future market outcomes, whereas positive sGovt, fGovt, sCorp and fCorp tend to be bad news.

It is noteworthy that some variables have very similar coefficient estimates for EMs and DMs (such as entropy, sGovt, and $s$ Corp), whereas others (like rate and artcount) only seems to matter in one group (rate for DM, and artcount for EM) but not in the other. The entropy measure, in particular, is associated with very consistent coefficient estimates between EMs and DMs in all four of our forecasting specifications.

\subsection{Trading Strategy based on Out-of-Sample Model Predictions}

To evaluate the economic importance of our text measures, we analyze how useful textual information would be to a mean-variance optimizing investor who already had access to our Baseline model's out-of-sample forecasts. In other words, we assume an investor forms at each time $t$ an estimate of future returns and volatility using the five variables that constitute our Baseline out-of-sample model. We then additionally allow this investor to condition, using only out-of-sample data, on our text measures. We refer to this as the $C M$ model. Finally, we also allow an investor to estimate next period's mean return and volatility for a given country using only historical return data. We refer to this as the Naïve model (this model is just a rolling country fixed effect).

Following Campbell and Thompson (CT, 2008), we assume a myopic mean-variance investor whose allocation to country $i$ at time $t$ is 


$$
w_{t}^{i}=\frac{E_{t}\left[r_{t+1}^{i}-r_{f}\right]}{\gamma \times \operatorname{var}_{t}\left(r_{t+1}^{i}-r_{f}\right)}
$$

This weight is applied to the month $t+1$ excess return of country $i$. Like Campbell and Thompson we cap $w_{t}^{i}$ at 1.5 , but unlike CT we allow short-selling by imposing a floor of -1.5 . A floor of zero makes sense in the CT setting because they analyze the allocation between cash and the stock market; but in our context, negative information in our signals about a given country's stock returns is useful and ought to be used in the trading strategy, which is feasible given our focus on country-level stock index trading. Furthermore, we set $\gamma=5$ (it is 3 in their paper) because with lower risk aversion our weights often hit the 1.5/-1.5 boundary rendering intermodel variation less important. Finally, to aggregate country weights into a portfolio at time $t$ we divide all $w_{t}^{i}$, s by the number of countries for which we have a time $t$ signal. These weights are applied to time $t+1$ returns. The net portfolio position is invested in the US 6-month T-bill.

The conditional moments in (8) are calculated using either the out-of-sample Naïve, Baseline or CM model (the latter two are estimated using the elastic net model). We use the model's 12-month ahead return estimate to proxy for the forward-looking monthly return expectation (estimating the model using one-month ahead returns does not identify the proper dependencies in the data because the time horizon is too short - as we discuss further below), and we use the square of the model's one-month ahead volatility estimate for the conditional variance (both quantities are reported in annualized terms). The portfolio is held for one month, and then reconstituted at time $t+1$ (to then realize time $t+2$ returns) based on the month $t+1$ ending information. Since we use a five-year window, our first portfolio is formed in April of 2003 and our last is formed in December of 2015. The aggregate amount invested varies 
between -0.5 and 1.4 times the portfolio capital, so the strategy we have parameterized is not overly levered. We run the strategy separately for our EM and DM countries.

We intentionally ignore portfolio level optimization (e.g., correlations across countries) and also employ (as do Campbell and Thompson) a myopic investment rule, to isolate the informational content of our text measures. Our approach follows DeMiguel, Garlappi and Uppal (2007) in using (i) rolling 5-year estimates of conditional moments to form myopic meanvariance portfolios, and (ii) an equal-weighted portfolio (in our case, equally weighted across countries though each country allocation varies according to equation (8)). We use overlapping 12-month observations to estimate how expected returns depend on our predictors in order to address the well-known problems of estimation error with using short-horizon returns. ${ }^{25}$ In fact, Britten-Jones et al. (2011) show that a predictive model with overlapping returns can be transformed into a predictive model for one-period ahead returns but with a properly transformed set of regressors. Our use of the untransformed 12-month ahead forecast in a monthly rebalanced myopic portfolio is certainly suboptimal, ${ }^{26}$ but is transparent and captures enough of the underlying structure in the data to lead to meaningful results.

To evaluate the economic significance of our results we estimate the three-factor international asset pricing model suggested by Brusa, Ramadorai, and Verdelhan (2017), henceforth BRV. Our factors are the net total return of the MSCI global index, the return on a currency carry trade, and the return on an investment in the US dollar funded by borrowing

\footnotetext{
${ }^{25}$ For example, Cochrane and Piazzesi (2005) claim on p. 139 that to see the underlying economic structure in their model for bond returns they can't use monthly observations, and must use overlapping annual ones. Also see Britten-Jones, Neuberger, and Nolte (2011).

${ }^{26}$ See Barberis (2000) for a comparison of myopic versus dynamic portfolio rules in the presence of return predictability.
} 
against a basket of global currencies. ${ }^{27}$ Table 16 shows the results of these regressions. The CM strategy generates lower market exposures than the Baseline and Naïve models. Both strategies have minimal exposures to the currency carry factor, and are short the dollar (which is a mechanical outcome of a net long in foreign stock markets without hedging the currency exposure). We note that for both EM and DM strategies, the Naïve strategy (i.e. rolling countrylevel means and volatilities) leads to very poor investment outcomes. The Baseline model is better, and leads to an economically significant $6.8 \%$ annual alpha for DM countries (in general, with a single series with 153 monthly observations we will not have much power against the null), although the Baseline model delivers a much weaker 3.27\% alpha for EM countries. The CM model, which augments the Baseline model with our text measures, is the best performer in both samples, with an annual alpha of $8.8 \%$ in each - this is a very large economic effect, though the alpha is statistically significant only in the EM sample.

Given our interest in the incremental information content of textual measures, perhaps the more interesting aspect of our analysis is not the absolute values of the alphas but whether the difference between the alphas of the CM and Baseline models is large. As Panel B of Table 16 shows, the difference is $2 \%$ per year for DMs and 5.5\% percent per year for EMs. Both differences are clearly important economically, and especially so for EM countries. Differences are also statistically significant at standard levels. ${ }^{28}$ This confirms our finding from the in-

\footnotetext{
${ }^{27}$ The currency carry trade and US dollar index return data are available from Lustig and Verdelhan's websites, but do not cover our entire sample. Instead, for the carry trade we use the Deutsche Bank Currency Carry USD Total Return Index, and for the US dollar index we use the US Dollar Index. The US Dollar Index is adjusted to have a negative $1.8 \%$ per year carry to match the average return of the US dollar index obtained from Lustig and Verdelhan. This adjusted dollar index and the Deutsche Bank carry trade index match the BRV factors very closely in the part of the sample where they overlap. The MSCI returns and both currency series are obtained from Bloomberg.

${ }^{28}$ The reason we have much more power to reject the null that the differences are zero is because the residuals from the CM and Baseline models are highly positively correlated (over 90\%) which leads to their difference having very little volatility.
} 
sample panels that our text-based measures yielded incrementally more predictive power for the EM countries. In a carefully constructed out-of-sample test we have shown that by using modern model selection techniques, we are able to use the information content of our text-based country level measures to meaningfully improve on investment performance.

\section{Conclusion}

We believe this is the first study of country-level stock returns and risks that relates news to future risk and return. We develop an atheoretical approach for capturing news through various word flow measures, including sentiment, frequency, unusualness (entropy), and the topical context in which these word flow outcomes occur. We apply that approach to 51 countries over the time period 1998 to 2015 . We find that it is possible to develop a parsimonious and flexible approach to extract from news flow information that is useful for forecasting equity market risk and returns. We find that news contained in our text flow measures forecasts one year ahead returns and drawdowns. One interpretation of this finding is that word flow captures "collective unconscious" aspects of news that are not understood at the time articles appear, but that capture influences on the market that have increasing relevance over time. It may be that these unconscious aspects of news even influence fundamental economic behavior in ways that produce changes in returns and risks, as conjectured by Shiller (2017).

We consider the importance of topical context by giving all news articles weights according to the topics they cover. Topical context is defined using the Louvain method for grouping words into clusters, or word groups. In our sample, there are five such topic clusters for EMs and five for DMs, four of which are common to both sets of countries. 
It is useful to divide news analysis of countries by considering EMs and DMs separately, because the basic statistical properties of news and returns are different for the two sets of countries, as are the relevant topics for news stories.

Principal components analysis of topic areas suggests a possible change in coefficient values occurs during the onset of the global financial crisis. We divide our sample period into two at February 2007 to take this change into account, and we find that coefficient values on various word flow measures do change over time.

Our word flow measures (sentiment, frequency, and entropy) capture important aspects of news that are relevant for returns, volatility, and drawdown risk, and have incremental predictive power over and above a Baseline specification of standard control variables. Coefficient magnitudes of text flow measures are often large. News tends to divide into good or bad news that is relevant both for returns or for risk (measured either by volatility or drawdown).

The predictive content of sentiment, frequency and entropy not only vary over time, but are also context-specific. Depending on the topic area of the article in which word flow appears, and the timing, some positive sentiment news days appear as negative news events.

Word flow measures tend to have greater incremental predictive power (measured in terms of percentage improvement in R-squared) for understanding returns and risks in EMs, although they also have important incremental predictive power for returns and drawdowns in DMs.

We compare the predictive power of our atheoretical approach to analyzing the word flow of news with the Baker, Bloom and Davis approach to measuring economic policy uncertainty through an a priori identification of key words. We find that our approach is 
correlated with the BBD measure. The BBD measure, however, has much less incremental explanatory power for returns, volatility and drawdown risk than our word flow measures, and in regressions that include both the $\mathrm{BBD}$ measure and our measures, the $\mathrm{BBD}$ measure loses statistical significance.

We perform out-of-sample testing using an elastic net regression to investigate whether our model is economically useful despite the large number of explanatory variables and the time variation in estimated coefficient parameters. From the standpoint of out-of-sample trading strategies, the additional alpha generated by using text flow measures is greater in EMs. For both DMs and EMs, text measures contribute significantly to improvements in out-of-sample forecasts relative to a Baseline model that excludes text measures.

We conclude that the meaning of news flow can be captured usefully through a small number of atheoretical measures (sentiment, frequency and entropy). The meaning of those measures for stock market risk and return vary over time, vary across EMs and DMs, and vary according to the topical context in which sentiment and frequency are measured. Thus, it is important to distinguish across country types and topical contexts, and permit coefficient estimates to vary over time, when using text to forecast risk and return. Nevertheless, we find that it is possible to construct a parsimonious and flexible forecasting model that maps usefully from these atheoretical, context-specific measures of news flow into equity market risk and return. 


\section{$\underline{\text { References }}$}

Ang, A. and G. Bekaert, 2007, Stock Return Predictability: Is it There? Review of Financial Studies, 20 (3), 651-707.

Angelidis, T. and N. Tessaromatis, 2017, Global Equity Country Allocation: An Application of Factor Investing, Financial Analysts Journal, 73 (4), 55-73.

Asness, C., J. Liew, and R. Stevens, 1997, Parallels Between the Cross-Sectional Predictability of Stock and Country Returns," Journal of Portfolio Management, 23 (3), 79-87.

Asness, C., T. Moskowitz, and L. Pedersen, 2013, Value and Momentum Everywhere. Journal of Finance, 68 (3), 929-985.

Baker, S. R., Bloom, N., and Davis, S. J., 2016, Measuring Economic Policy Uncertainty, Quarterly Journal of Economics, 131 (4), 1593-1636.

Barberis, N., 2000, Investing for the Long Run when Returns Are Predictable, Journal of Finance, 55 (1), 225-264.

Beim, D. O., and Calomiris, C. W., 2001, Emerging Financial Markets. New York: IrwinMcGraw Hill.

Bekaert, G., Erb, C., Harvey, C. and Viskanta, T. 1998, Distributional Characteristics of Emerging Market Returns and Asset Allocation, Journal of Portfolio Management, Winter, 102116.

Bekaert, G. and M. Hoerova, 2014, The VIX, the Variance Premium and Stock Market Volatility, Journal of Econometrics, 183, 181-192.

Blei, D., Ng, A. and Jordan, M., 2003, Latent Dirichlet Allocation. Journal of Machine Learning Research, 3, 993-1022.

Blondel, V., Guillaume, J.-L., Lambiotte, R., and Lefebvre, E., 2008, Fast Unfolding of Communities in Large Networks, Journal of Statistical Mechanics, 10, 10008.

Britten-Jones, M., Neuberger, A. and Nolte, I., 2011, Improved Inference in Regression with Overlapping Observations. Journal of Business Finance \& Accounting, 38, 657-683.

Brusa, F., Ramadorai, T., and Verdelhan, A., 2017, The International CAPM Redux, Working Paper, Oxford University.

Calomiris, C. W., Love, I., and Martinez Peria, M.S., 2012, Global Returns' Sensitivities to Crisis Shocks, Journal of International Money and Finance, 30, 1-23.

Calvo, G. A., and Reinhart, C. M., 2002, Fear of Floating, Quarterly Journal of Economics 117 (2), 379-408. 
Campbell, J. and Thompson, S., 2008, Predicting Excess Stock Returns Out of Sample: Can Anything Beat the Historical Average? Review of Financial Studies, 21 (4), 1509-1531.

Cochrane, J. and Piazzesi, M., 2005. Bond Risk Premia, American Economic Review, 95 (1), $138-160$.

Cruces, J. J., and Trebesch, C. 2013. Sovereign Defaults: The Price of Haircuts, American Economic Journal: Macroeconomics, 5 (3), 85-117.

Das, S.R., and Chen, M.Y., 2007, Yahoo! For Amazon: Sentiment Extraction from Small Talk on the Web, Journal of Management Science, 53(9) 1375-1388.

DeMiguel, V., Garlappi, L., and Uppal, R., 2007, Optimal Versus Naive Diversification: How Inefficient Is the 1/N Portfolio Strategy? The Review of Financial Studies, 22 (5), 1915-1953.

Fama, E., Fisher, L., Jensen, M. and Roll, R., 1969, The Adjustment of Stock Prices to New Information, International Economic Review, 10(1), 1-21.

Fama, E. and K. French, 1988, Dividend Yields and Expected Stock Returns, Journal of Financial Economics, 22, 3-25.

Froot, K., X. Lou, G. Ozik, R. Sadka, and S. Shen, 2017, Media Reinforcement in International Financial Markets, working paper.

Garcia, D. 2013. Sentiment during Recessions, Journal of Finance, 68(3), 1267-1300.

Glasserman, P., and Mamaysky, H., 2016a., Does Unusual News Forecast Market Stress? OFR Working Paper 16-04, April 20.

Glasserman, P., and Mamaysky, H., 2016b, Market Efficiency with Micro and Macro Information, Working Paper, Columbia University.

Ghysels, E., Plazzi, A., and Valkanov, R. 2016. Why Invest in Emerging Markets? The Role of Conditional Return Asymmetry, Journal of Finance 71 (5), 2145-2191.

Hastie, T. and Qian, J., 2016, Glmnet Vignette, Working Paper, Stanford University.

Hendershott, T., Livdan, D., and Schurhoff, N., 2015, Are Institutions Informed about News? Journal of Financial Economics, 117, 249-287.

Heston, S. and Sinha, N., 2017, News vs. Sentiment: Predicting Stock Returns from News Stories, Financial Analysts Journal, 73 (3), 67-83.

Hjalmarsson, E., 2010, Predicting Global Stock Returns," Journal of Financial and Quantitative Analysis, 45 (1), 49-80. 
Kaminsky, G., and Schmukler, S., 2008, Short-Run Pain, Long-Run Gain: The Effects of Financial Liberalization, Review of Finance, 12(2), 253-292.

Karolyi, G. A., 2015, Cracking the Emerging Markets Enigma. New York: Oxford University Press.

La Porta, R., Lopez de Silanes, F., Shleifer, A., and Vishny, R., 1998, Law and Finance, Journal of Political Economy, 106 (6), 1113-1155.

Loughran, T., and McDonald, B., 2011, When Is a Liability not a Liability? Textual Analysis, Dictionaries, and 10-Ks, Journal of Finance 66 (1), 35-65.

Meila, M., 2007, Comparing Clusterings - An Information Based Distance, Journal of Multivariate Analysis, 98, 873-895.

Netzer, O., Feldman, R., Goldenberg, J., and Fresko, M., 2012, Mine Your Own Business: Market Structure Surveillance Through Text Mining, Marketing Science, 31 (3), 1-23.

Newman, M.E.J. 2006. Modularity and Community Structure in Networks, Proceedings of the National Academy of Sciences, 103 (23), 8577-8582.

Newman, M.E.J. and Girvan, M., 2004, Finding and Evaluating Community Structure in Networks, Physical Review E, 69, 026113.

Nickell, S., 1981, Biases in Dynamic Models with Fixed Effects," Econometrica, 49 (6), $1417-1426$.

Richards, A., 1997, Winner-Loser Reversals in National Stock Market Indices: Can They be Explained? Journal of Finance, 52 (5), 2129-2144.

Roll, R., 1984, Orange Juice and Weather, American Economic Review, 74 (5), 861-880.

Rule, A., Cointet, J.-P. and Bearman, P.S., 2015, Lexical shifts, substantive changes, and continuity in State of the Union discourse, 17902014, Proceedings of the National Academy of Sciences, 112 (35), 1790-2014.

Shapiro, A., M. Sudhof, and D. Wilson, 2018, Measuring News Sentiment, FRBSF Working Paper 2017-01.

Shiller, R. J., 2017, Narrative economics. Presidential Address at the $129^{\text {th }}$ Annual Meeting of the American Economic Association.

Sinha, N. R., 2016, Underreaction to News in the US Stock Market, Quarterly Journal of Finance, 6 (2), 165000501-165000546. 
Stambaugh, R., 1999, Predictive Regressions, Journal of Financial Economics, 54, 375-421.

Tetlock, P. C., 2007, Giving Content to Investor Sentiment: The Role of Media in the Stock Market, Journal of Finance 62 (3), 1139-1168.

Tetlock, P. C., Saar-Tsechansky, M., and Macskassy, S., 2008, More Than Words: Quantifying Language to Measure Firms' Fundamentals, Journal of Finance, 63 (3), 1437-1467.

Tetlock, P. C., 2010, Does Public Financial News Resolve Asymmetric Information? Review of Financial Studies 23 (9), 3520-3557.

Tetlock, P. C., 2011, All the News That's Fit to Reprint: Do Investors React to Stale Information? Review of Financial Studies 24 (5), 1481-1512.

Thompson, S., 2011, Simple Formulas for Standard Errors that Cluster by Both Firm and Time, Journal of Financial Economics, 99, 1-10.

Thorsrud, L.A., 2016, Nowcasting Using News Topics: Big Data versus Big Bank, Working Paper, Norges Bank.

Tibshirani, R., 1996, Regression Shrinkage and Selection via the Lasso, Journal of the Royal Statistical Society, Series B, 58 (1), 267-288. 
Wordcloud

Mkt \# words $(\mathrm{mm})=236.23$
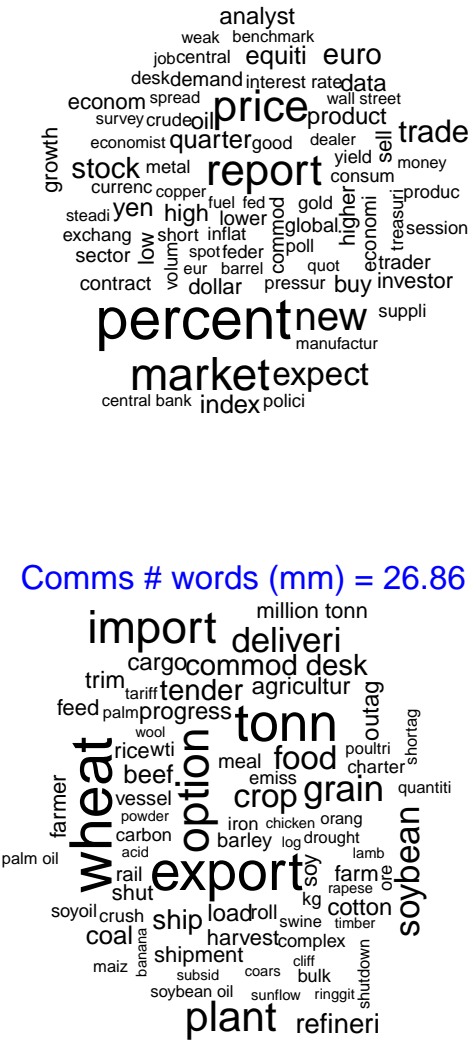

Govt \# words $(\mathrm{mm})=111.02$

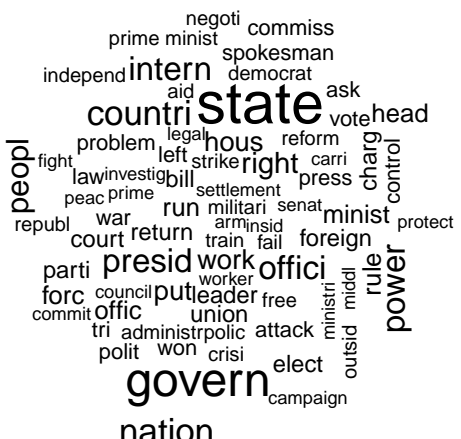

Credit \# words $(\mathrm{mm})=139.63$

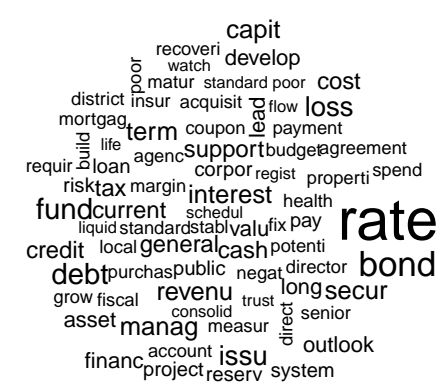

Corp \# words $(\mathrm{mm})=131.95$

compani

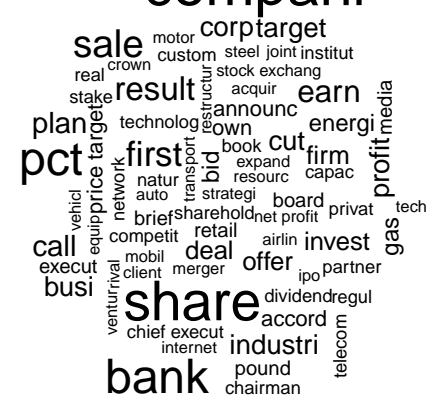

Figure 1: Word cloud plots for topics extracted from the developed markets corpus using the Louvain clustering algorithm. Each cluster shows the number of occurrences (in millions) of its words in the corpus. 
Wordcloud

Mkt \# words $(\mathrm{mm})=113.80$

percent

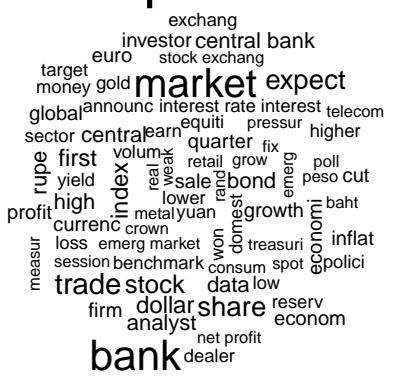

Comms \# words $(\mathrm{mm})=58.90$

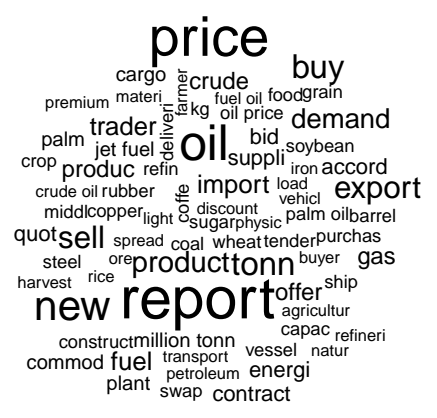

Govt \# words $\underset{\text { elect }}{(\mathrm{mm})}=76.58$

parliament

countr

build
brisi work
control

housfederpolit offici forc peopl

housfeder polik offici forc prime ministinternarm ask
vote protest fail
strime public polic roop law olland war put run prime

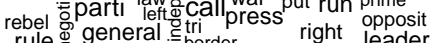

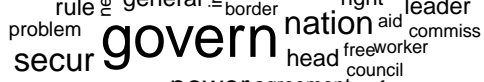
union power agreement reform return ministagenc carri

state ${ }_{\substack{\text { peac militar } \\ \text { spokesman }}}$

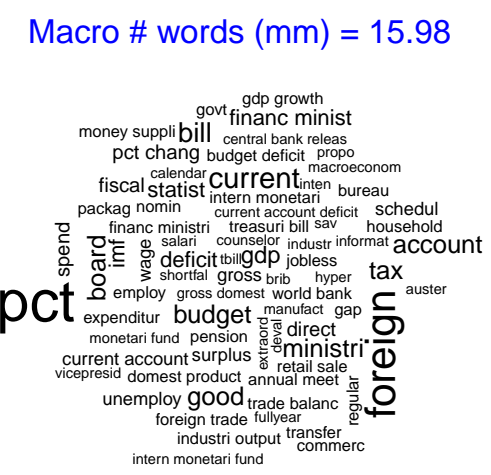

Corp \# words $(\mathrm{mm})=58.77$

compani invest debt infrastructur

rate $\begin{gathered}\text { asset } \\ \text { option }\end{gathered}$

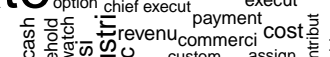

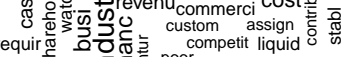

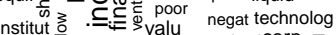

mstitut o seniorprojectstandardcorp o

matur plandevelop term airlin own

त partner system term airlin

lead margin issu motor $\mathbb{E}_{\text {co }}$ corpor

potentiloan privat capit

manag ${ }^{\text {credit deposit }}$.

local long

Figure 2: Word cloud plots for topics extracted from the emerging markets corpus using the Louvain clustering algorithm. Each cluster shows the number of occurrences (in millions) of its words in the corpus. 
Emerging markets

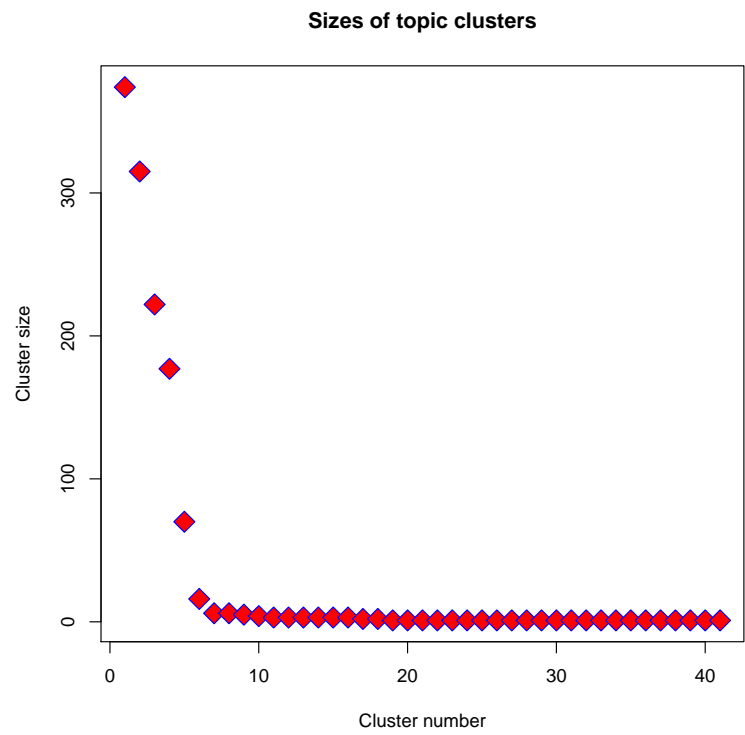

Developed markets

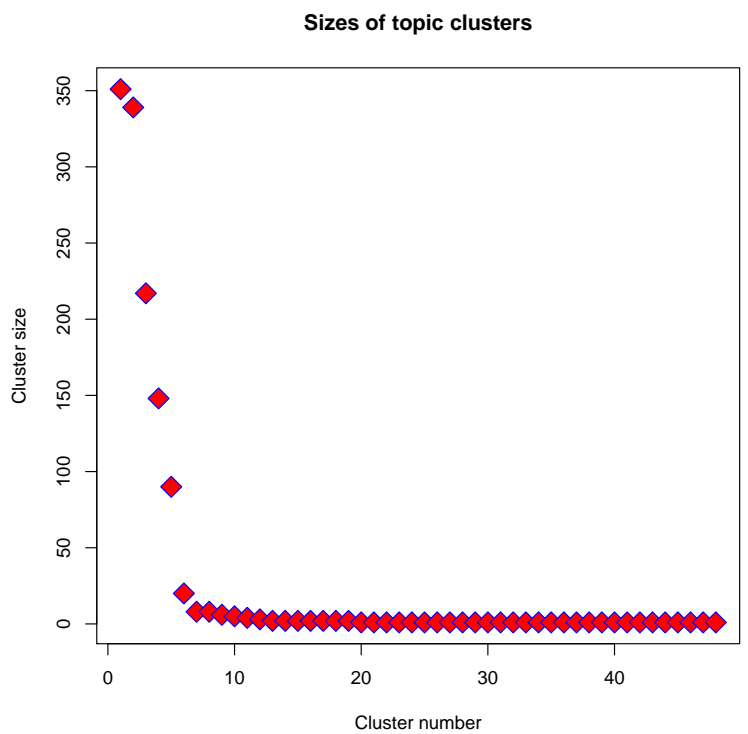

Figure 3: Number of words in original set of clusters identified by the Louvain algorithm for the emerging and developed markets corpora respectively. 
Factors decomposition of news topic sentiment in emerging markets

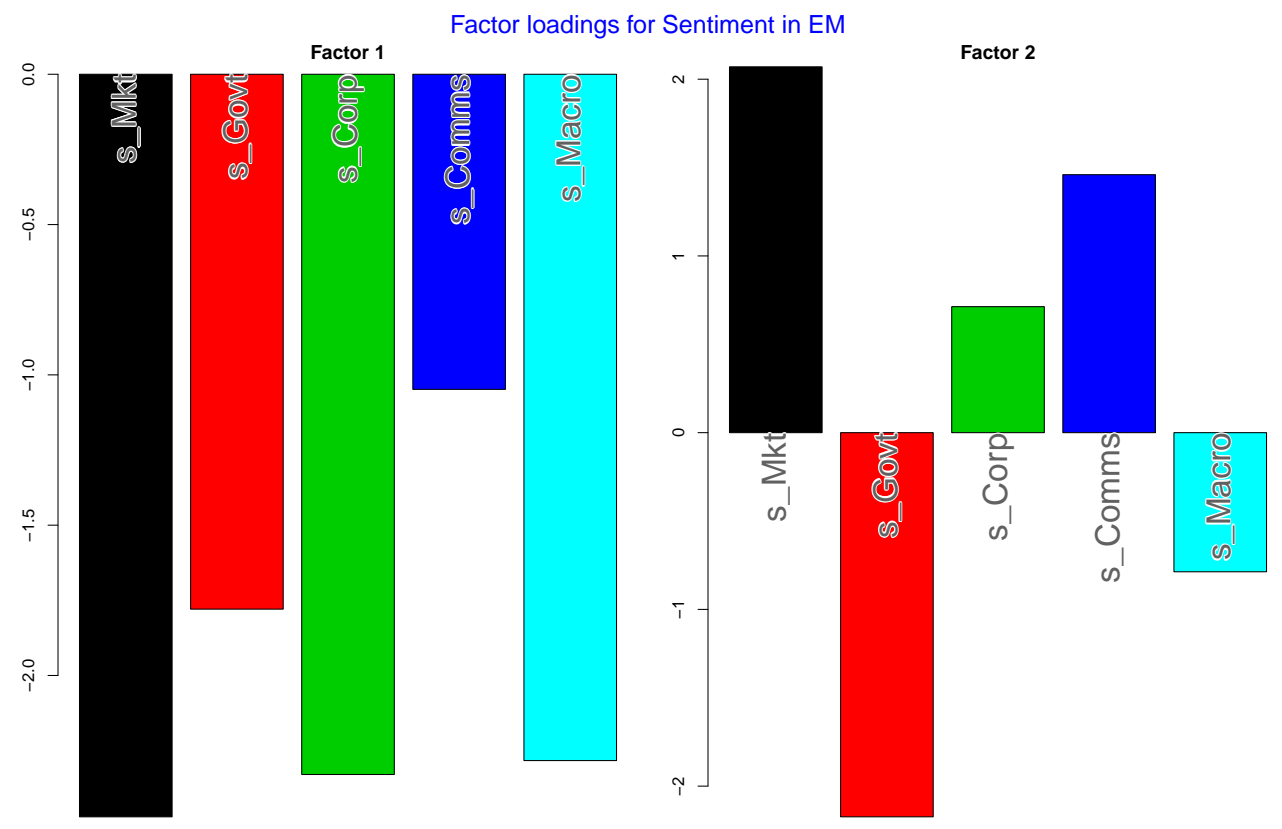

Factors for Sentiment in EM
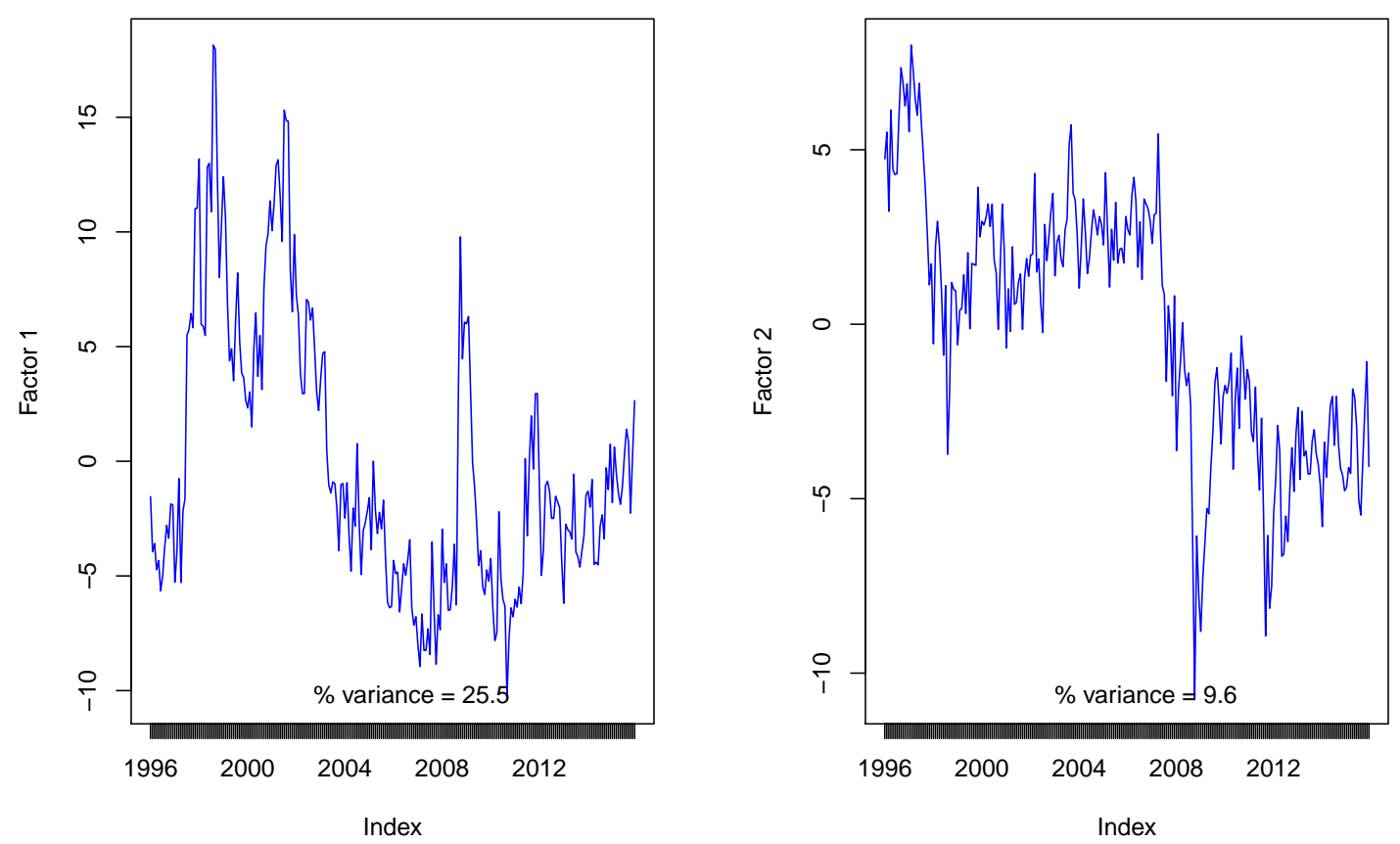

Figure 4: The figure shows the top factors from a principal components analysis of all country-topic sentiment series (i.e. \#(countries) $\times \#($ topics $)$ ) from emerging market countries. The top row shows the topic loadings of each factor. All country-topic sentiment series were normalized to unit variance. 
Factors decomposition of news topic sentiment in developed markets

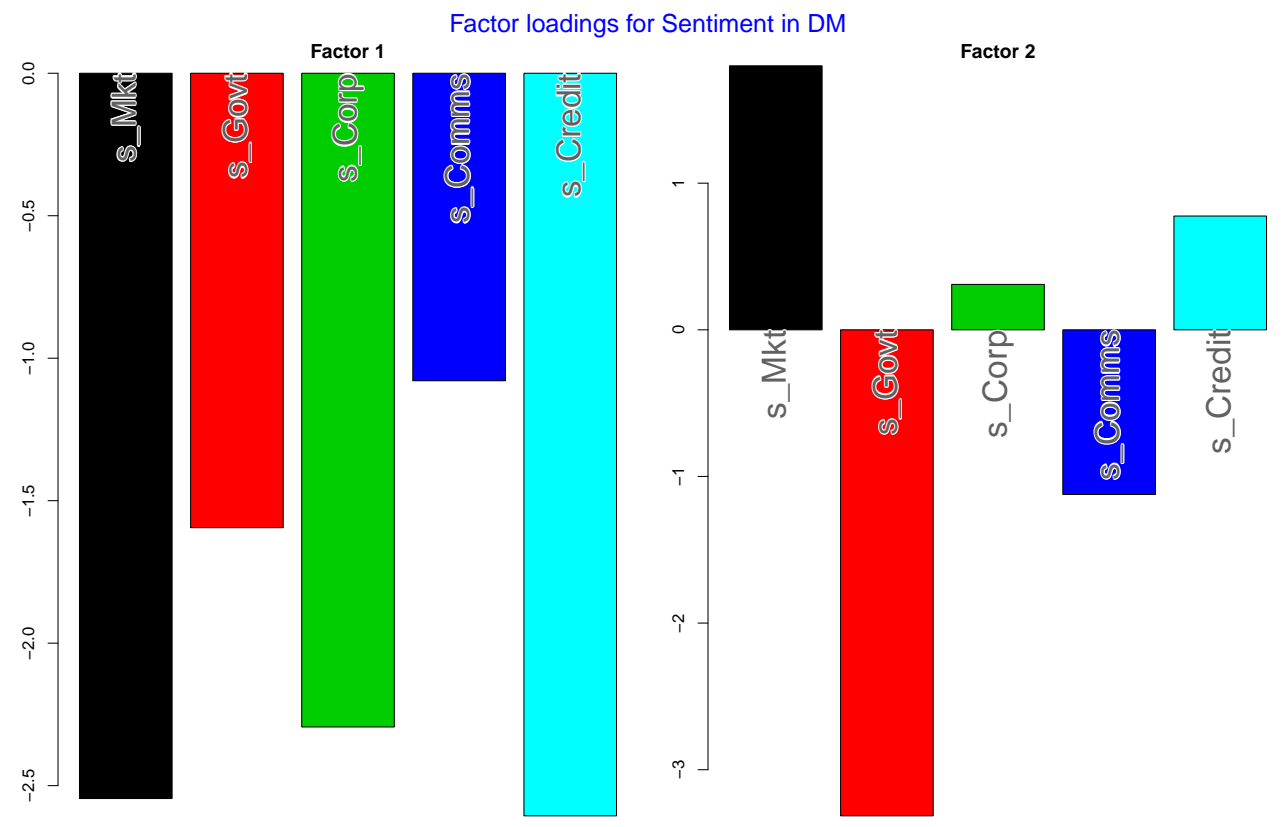

Factors for Sentiment in DM
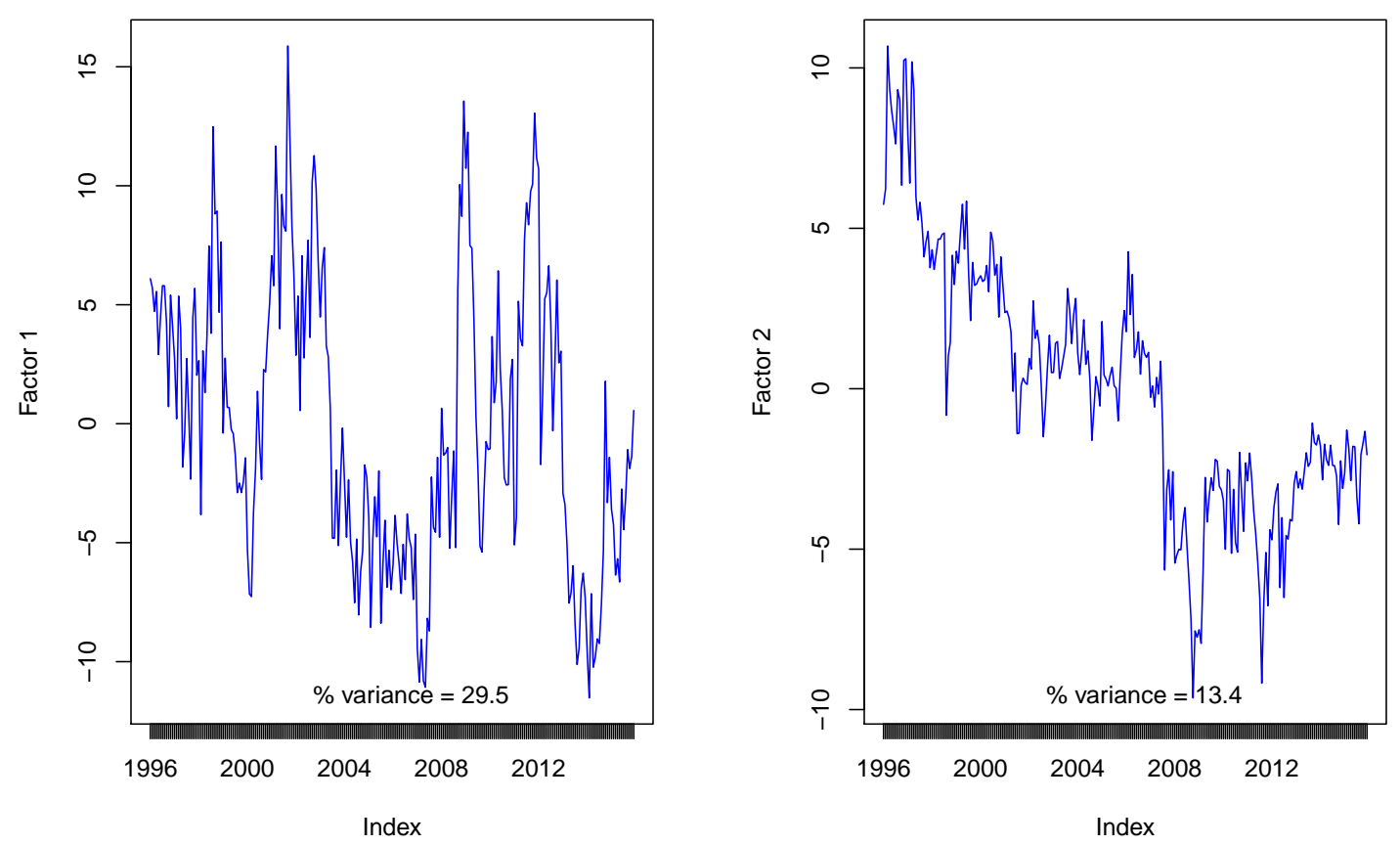

Figure 5: The figure shows the top factors from a principal components analysis of all country-topic sentiment series (i.e. \#(countries) $\times \#($ topics $)$ ) from developed market countries. The top row shows the topic loadings of each factor. All country-topic sentiment series were normalized to unit variance. 


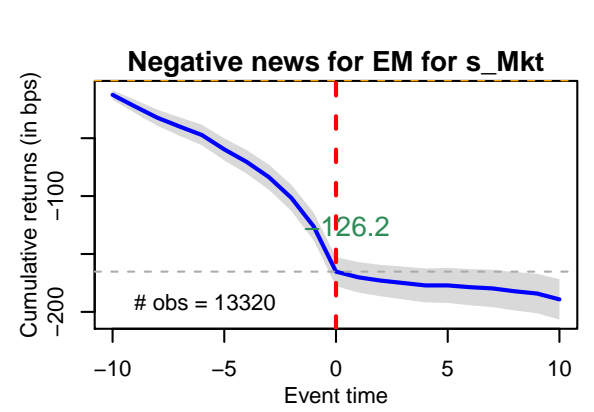

Event study for cumulative returns for EM
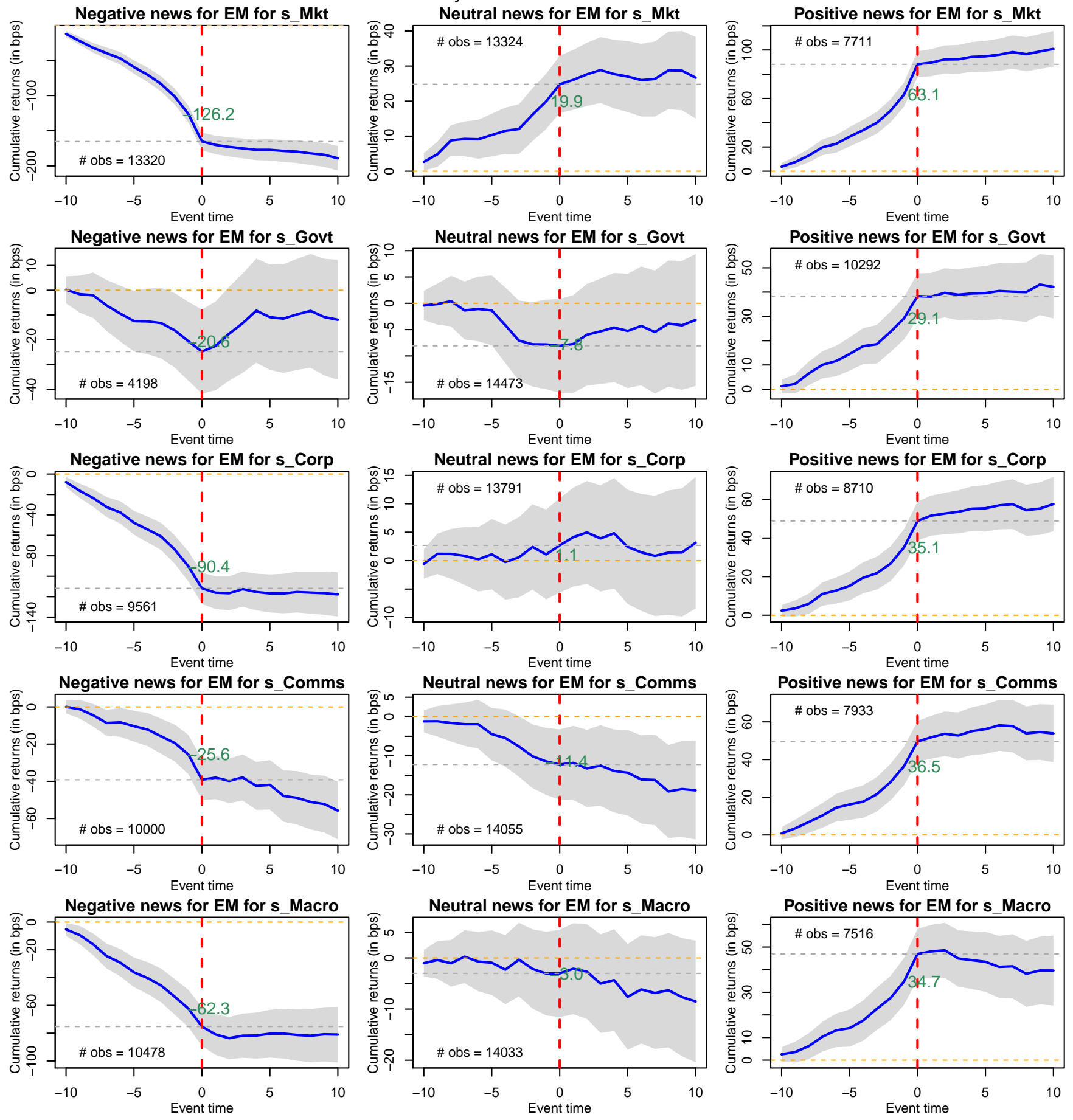

Figure 6: Event studies of cumulative abnormal returns on days that are in the bottom, middle (45\%-55\%) and top deciles by sentiment for each topic. Abnormal returns are the residuals from a regression of US dollar country index returns on a EM market index and a constant. Cumulative returns are shown in basis points, with two standard error bands. The cumulative return on the day prior to the event is labeled. The number of events in each study is shown on the plot. Events runs from Jan 1, 1996 to Dec 31, 2015. 


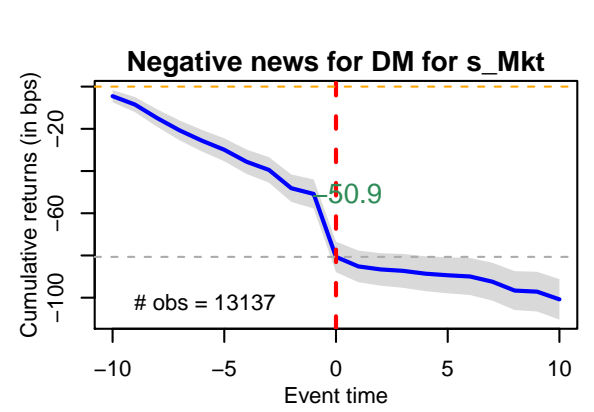

Event study for cumulative returns for DM
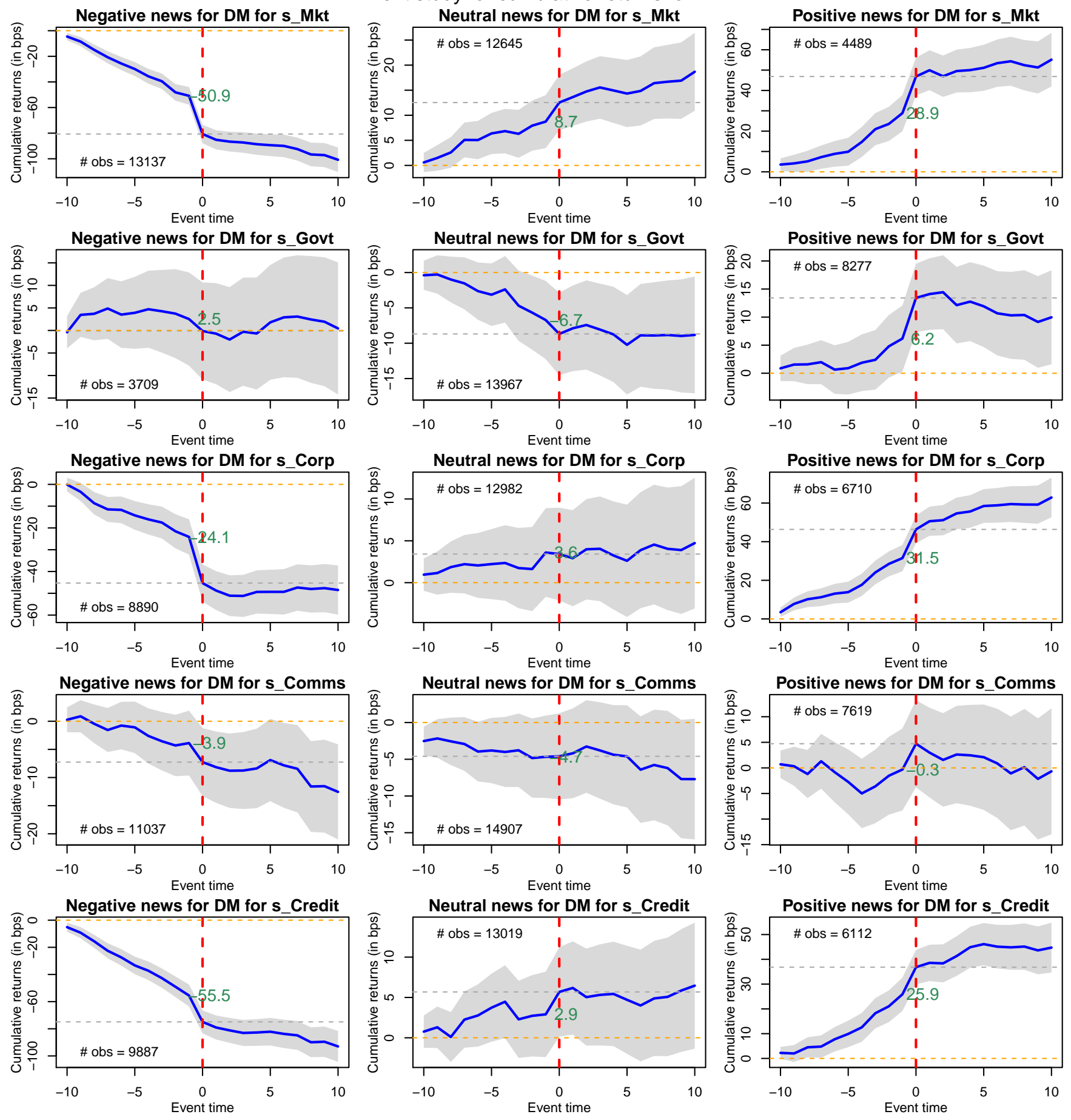

Figure 7: Event studies of cumulative abnormal returns on days that are in the bottom, middle (45\%-55\%) and top deciles by sentiment for each topic. Abnormal returns are the residuals from a regression of US dollar country index returns on a DM market index and a constant. Cumulative returns are shown in basis points, with two standard error bands. The cumulative return on the day prior to the event is labeled. The number of events in each study is shown on the plot. Events runs from Jan 1, 1996 to Dec 31, 2015. 


\section{How high entropy days are different for EM}
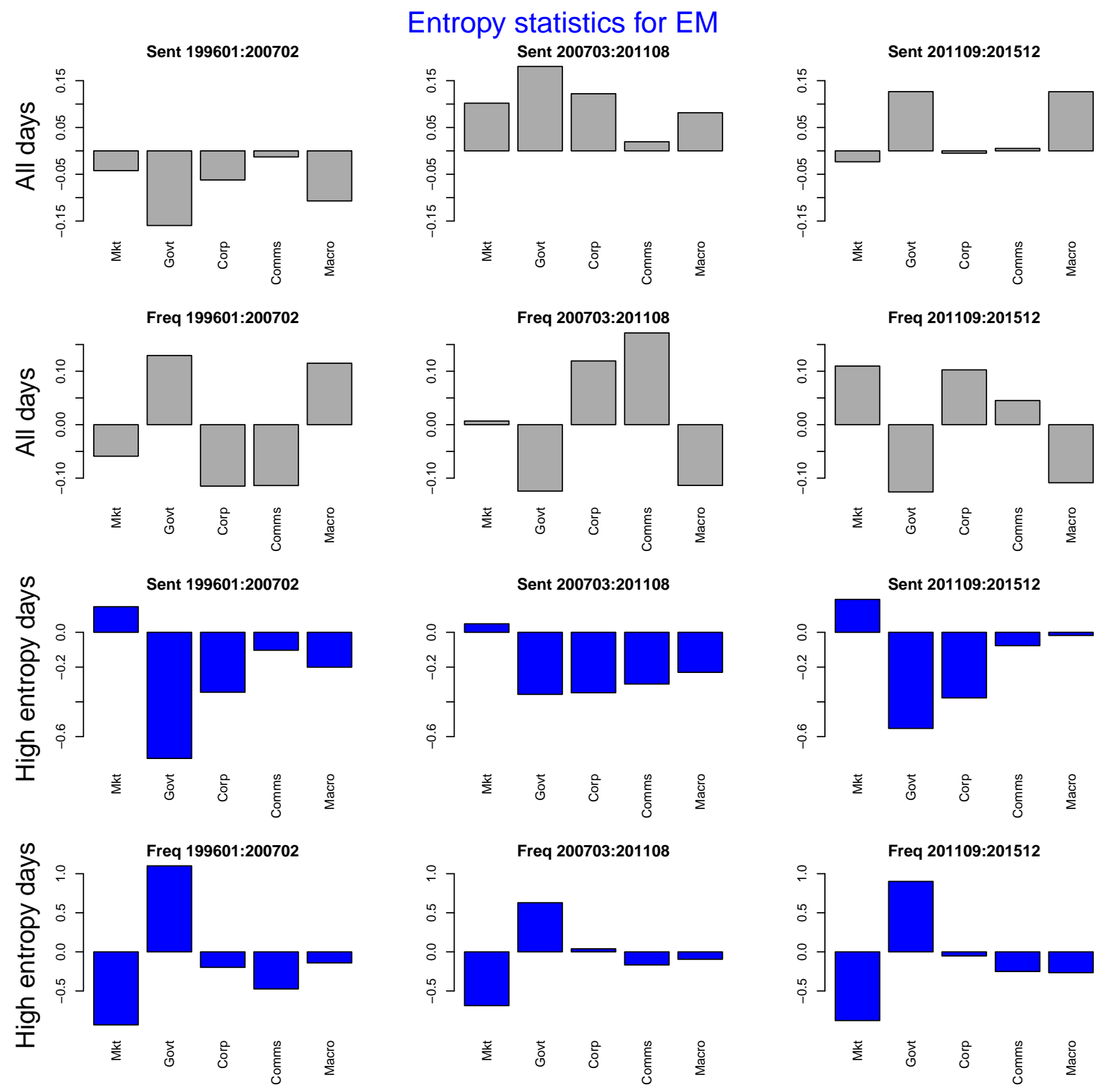

Figure 8: The sample is split into three time periods - pre-crisis, crisis, and post-crisis. Within each subperiod, the first row shows: the average sentiment by topic in that subsample minus the full-sample topic mean, divided by the full sample standard deviation of topic sentiment. The second row shows the same calculation but for topic frequency. The third row shows the same measure (sentiment) as in row 1 but restricted to country/day observations in the top $5 \%$ by entropy. The fourth row shows the same measure (frequency) as in row 2 but again for only the top $5 \%$ of country/days by entropy. 


\section{How high entropy days are different for DM}
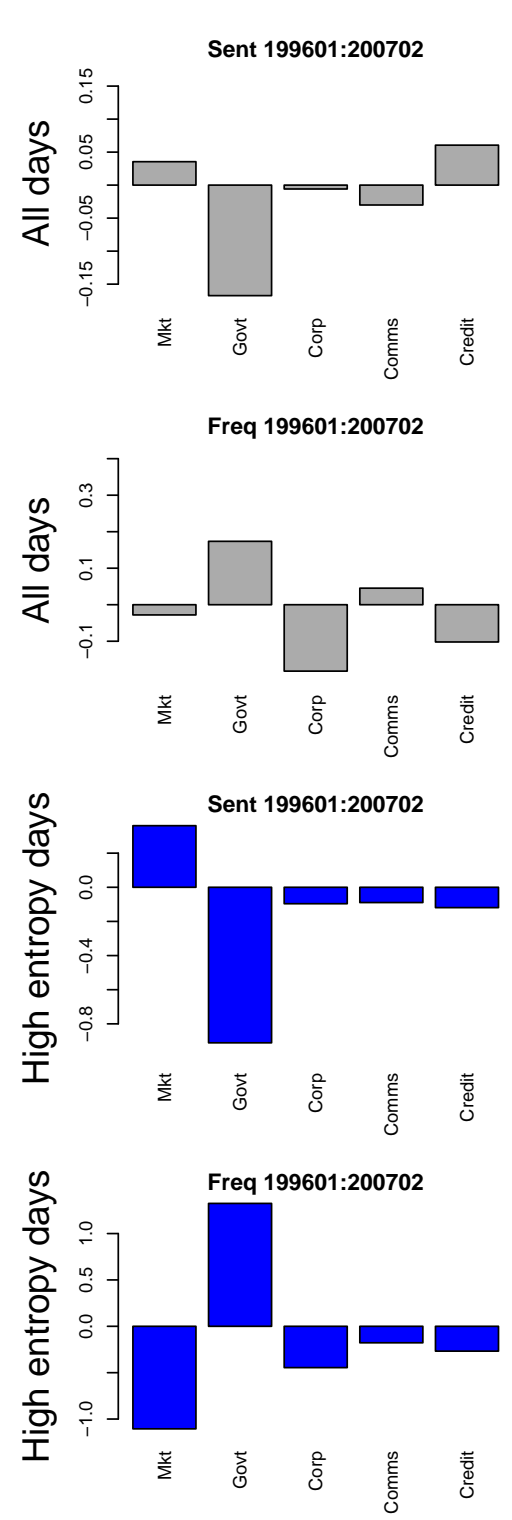

\section{Entropy statistics for DM}

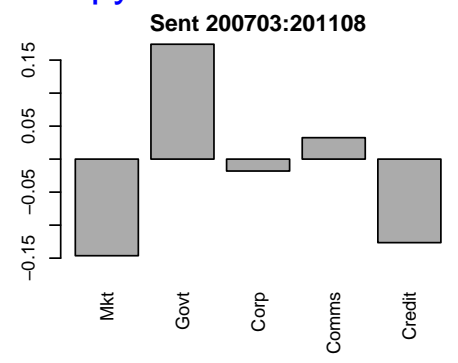

Freq 200703:201108

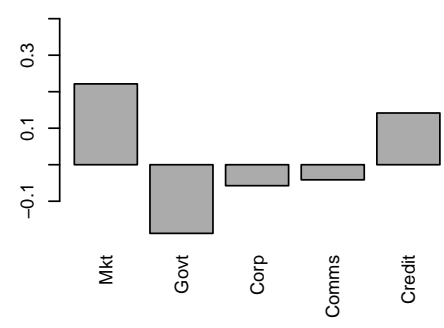

Sent 200703:201108

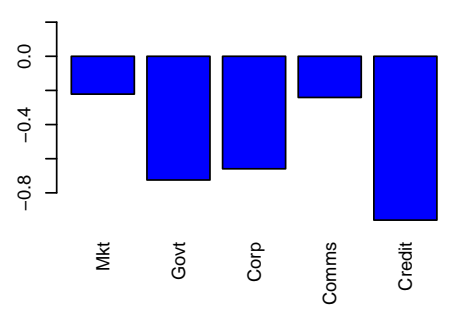

Freq 200703:201108

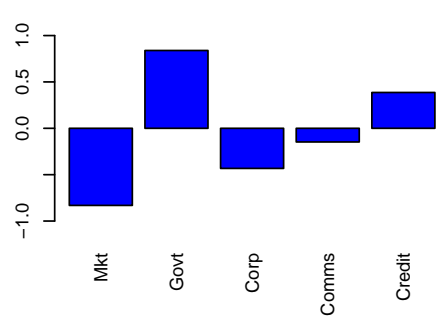

Sent 201109:201512

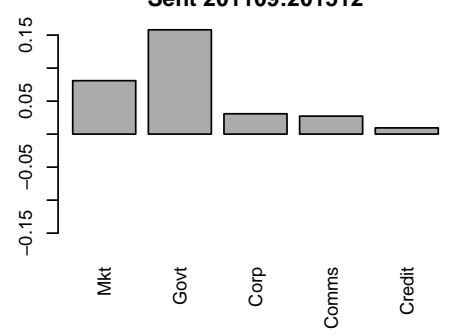

Freq 201109:201512

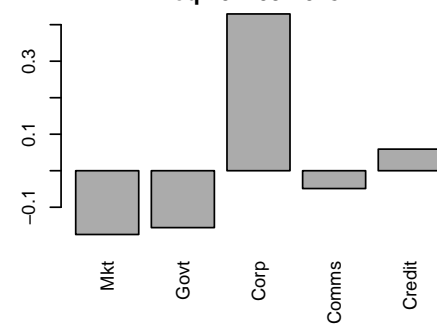

Sent 201109:201512

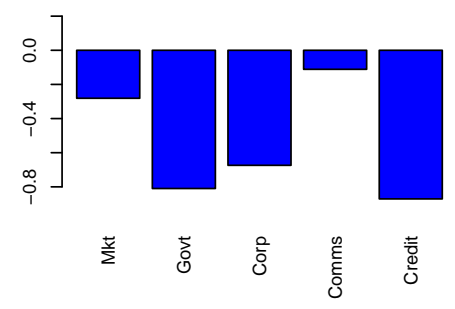

Freq 201109:201512

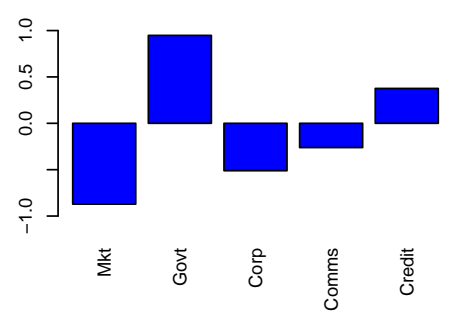

Figure 9: The sample is split into three time periods - pre-crisis, crisis, and post-crisis. Within each subperiod, the first row shows: the average sentiment by topic in that subsample minus the full-sample topic mean, divided by the full sample standard deviation of topic sentiment. The second row shows the same calculation but for topic frequency. The third row shows the same measure (sentiment) as in row 1 but restricted to country/day observations in the top $5 \%$ by entropy. The fourth row shows the same measure (frequency) as in row 2 but again for only the top $5 \%$ of country/days by entropy. 
Coefficient time series from elastic net for 12 month returns

Coefficients for return12 DM and EM countries
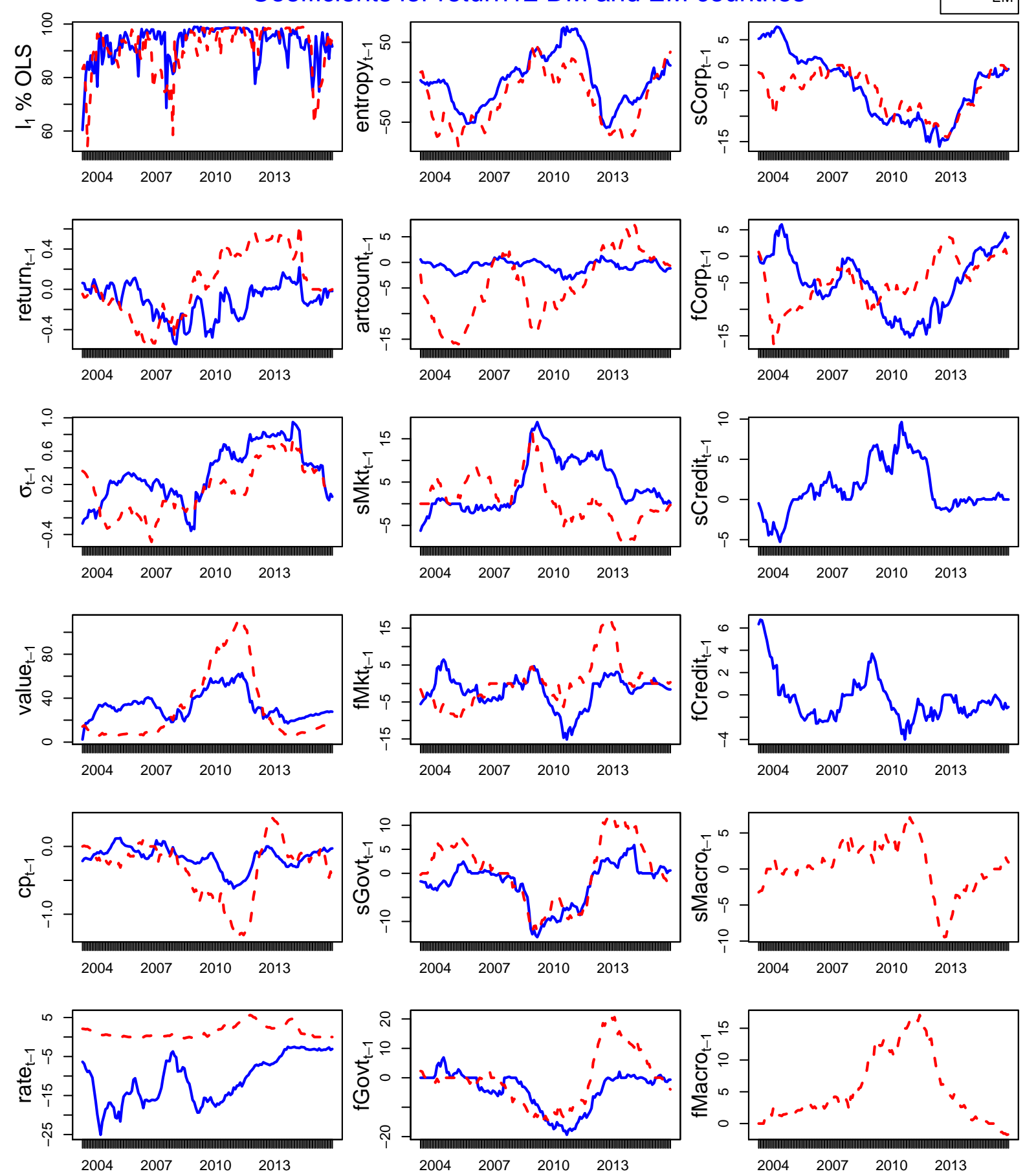

Figure 10: The charts show the time series of coefficient estimates from a rolling elastic net regression to forecast 12 month returns. The chart labeled " $l_{1} \%$ of OLS" gives the ratio of the elastic net coefficient $l_{1}$-norm to the OLS coefficient $l_{1}$-norm in every time period. The elastic net regressions are run over rolling 60 -month windows, with weighting parameter chosen to minimize cross-validation error. The penalty is 0.75 elastic net and 0.25 ridge regression. 
Coefficient time series from elastic net for next 12 month drawdown
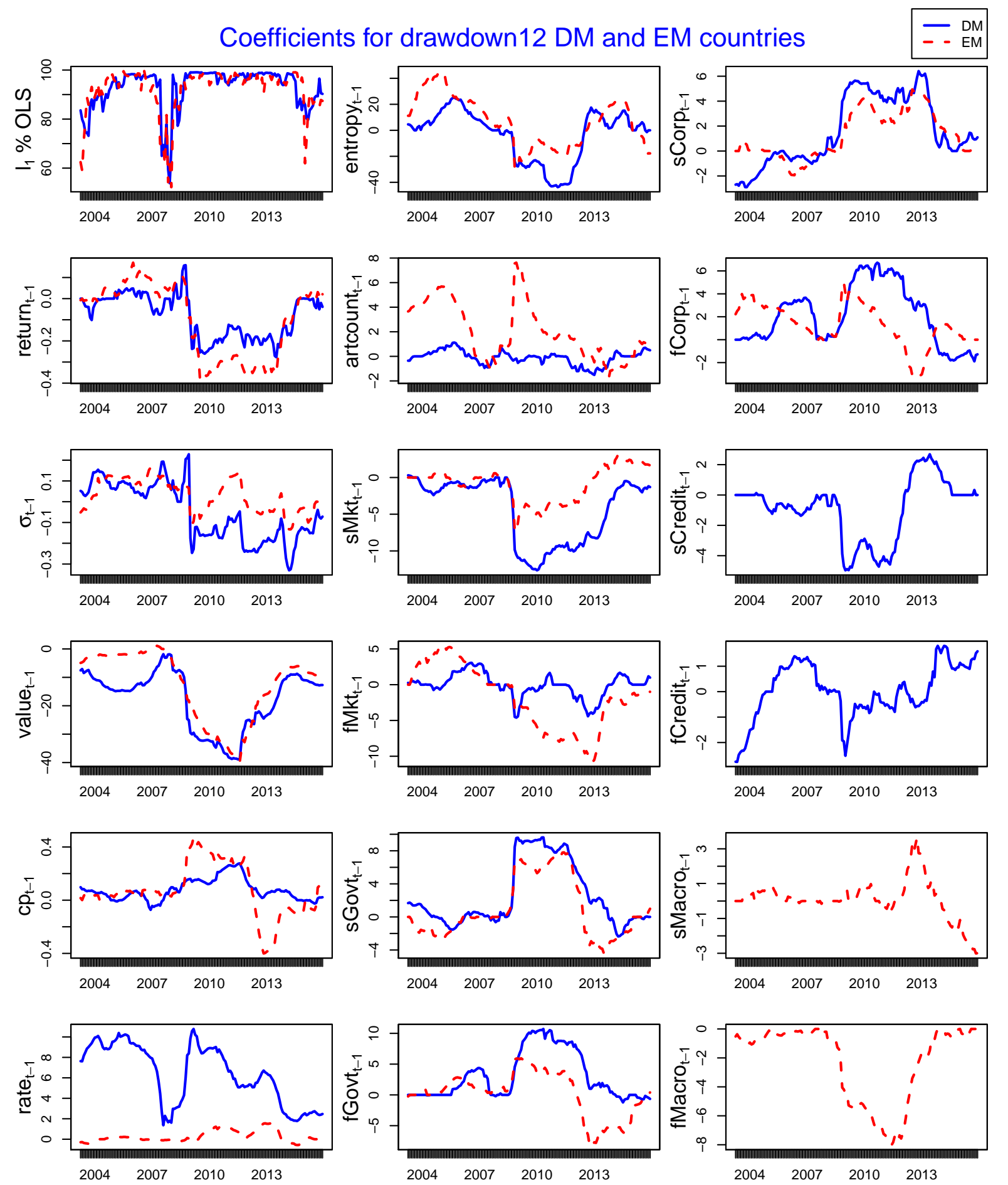

Figure 11: The charts show the time series of coefficient estimates from a rolling elastic net regression to forecast next 12 month drawdown. The chart labeled " $l_{1} \%$ of OLS" gives the ratio of the elastic net coefficient $l_{1}$-norm to the OLS coefficient $l_{1}$-norm in every time period. The elastic net regressions are run over rolling 60 -month windows, with weighting parameter chosen to minimize cross-validation error. The penalty is 0.75 elastic net and 0.25 ridge regression. 
Coefficient time series from elastic net for realized volatility Coefficients for sigma DM and EM countries
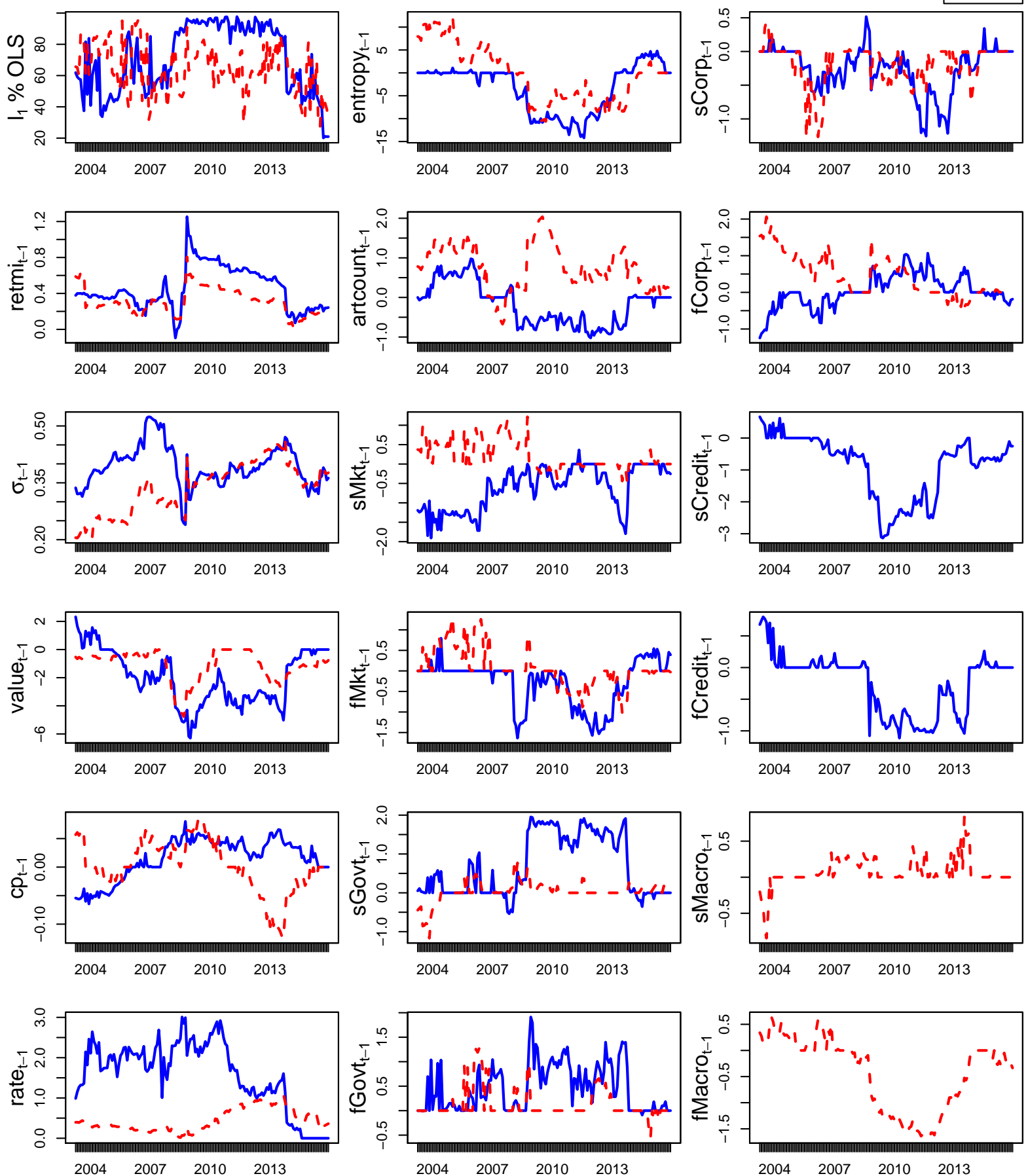

Figure 12: The charts show the time series of coefficient estimates from a rolling elastic net regression to forecast realized volatility. The chart labeled " $l_{1} \%$ of OLS" gives the ratio of the elastic net coefficient $l_{1}$-norm to the OLS coefficient $l_{1}$-norm in every time period. The elastic net regressions are run over rolling 60 -month windows, with weighting parameter chosen to minimize cross-validation error. The penalty is 0.75 elastic net and 0.25 ridge regression. 
List of EM and DM countries

\begin{tabular}{|c|c|c|c|c|c|c|c|}
\hline \multicolumn{4}{|c|}{ EM Countries } & \multicolumn{4}{|c|}{ DM Countries } \\
\hline & Country & BBG Index & TR Code & & Country & BBG Index & TR Code \\
\hline 1 & Argentina & BURCAP & $\mathrm{AR}$ & 1 & Australia & AS52 & $\mathrm{AU}$ \\
\hline 2 & Brazil & IBOV & $\mathrm{BR}$ & 2 & Austria & ATX & AT \\
\hline 3 & Chile & IGPA & CL & 3 & Belgium & BELPRC & $\mathrm{BE}$ \\
\hline 4 & China (PRC) & SHCOMP & $\mathrm{CN}$ & 4 & Canada & SPTSX & $\mathrm{CA}$ \\
\hline 5 & Colombia & COLCAP & $\mathrm{CO}$ & 5 & Denmark & $\mathrm{KAX}$ & DK \\
\hline 6 & Czech Republic & PX & $\mathrm{CZ}$ & 6 & $\mathrm{DM}$ & MXWO & - \\
\hline 7 & EM & MXEF & EMRG & 7 & Finland & HEX & FI \\
\hline 8 & Estonia & TALSE & $\mathrm{EE}$ & 8 & France & $\mathrm{CAC}$ & FR \\
\hline 9 & Ghana & GGSECI & $\mathrm{GH}$ & 9 & Germany & DAX & $\mathrm{DE}$ \\
\hline 10 & Hong Kong & HSI & HK & 10 & Greece & ASE & GR \\
\hline 11 & Hungary & BUX & $\mathrm{HU}$ & 11 & Iceland & ICEXI & IS \\
\hline 12 & India & SENSEX & IN & 12 & Ireland & ISEQ & IE \\
\hline 13 & Indonesia & JCI & ID & 13 & Italy & ITLMS & IT \\
\hline 14 & Israel & TA-25 & IL & 14 & Japan & NKY & JP \\
\hline 15 & Kenya & NSEASI & $\mathrm{KE}$ & 15 & Luxembourg & LUXXX & $\mathrm{LU}$ \\
\hline 16 & Malaysia & FBMKLCI & MY & 16 & Netherlands & AEX & NL \\
\hline 17 & Mexico & INMEX & MX & 17 & New Zealand & NZSE & NZ \\
\hline 18 & Nigeria & NGSEINDX & $\mathrm{NG}$ & 18 & Norway & OSEBX & $\mathrm{NO}$ \\
\hline 19 & Peru & SPBL25PT & $\mathrm{PE}$ & 19 & Portugal & BVLX & PT \\
\hline 20 & Philippines & PCOMP & $\mathrm{PH}$ & 20 & Singapore & STI & SG \\
\hline 21 & Poland & WIG20 & PL & 21 & Spain & IBEX & $\mathrm{ES}$ \\
\hline 22 & Russia & INDEXCF & $\mathrm{RU}$ & 22 & Sweden & OMX & $\mathrm{SE}$ \\
\hline 23 & Slovakia & SKSM & SK & 23 & Switzerland & SPI & $\mathrm{CH}$ \\
\hline 24 & Slovenia & SBITOP & SQ & 24 & United Kingdom & UKX & GB \\
\hline 25 & South Africa & JALSH & $\mathrm{ZA}$ & 25 & United States & SPX & US \\
\hline 26 & South Korea & KOSPI & $\mathrm{KR}$ & & & & \\
\hline 27 & Thailand & SET50 & $\mathrm{TH}$ & & & & \\
\hline 28 & Turkey & XU100 & $\mathrm{TR}$ & & & & \\
\hline 29 & Ukraine & PFTS & $\mathrm{UA}$ & & & & \\
\hline
\end{tabular}

Table 1: List of EM and DM countries and their associated stock market index from Bloomberg ("BBG Index") as well as their country code ("TR Code") in the ThomsonReuters Machine Readable News archive. The EM and DM rows refer to the MSCI EM and DM indexes respectively, which are used as the market benchmark in several sections of the paper. Iceland is used in our event studies and in the topic clustering analysis, but is excluded from all our panel and out-of-sample forecasting analysis. 
Similarity of developed and emerging market clusters

\begin{tabular}{rrrrrr}
\hline & Mkt (EM) & Govt (EM) & Corp (EM) & Comms (EM) & Macro (EM) \\
\hline Mkt (DM) & 0.59 & 0.01 & 0.01 & 0.13 & 0.05 \\
Govt (DM) & 0.01 & 0.82 & 0.01 & 0.01 & 0.04 \\
Corp (DM) & 0.10 & 0.02 & 0.23 & 0.07 & 0.04 \\
Comms (DM) & 0.01 & 0.01 & 0.03 & 0.33 & 0.21 \\
Credit (DM) & 0.04 & 0.04 & 0.46 & 0.03 & 0.06 \\
\hline
\end{tabular}

Table 2: Comparison of overlap between developed and emerging market clusters obtained via the Louvain network algorithm. For two clusters, $A$ and $B$, the corresponding entry in the table reports $\#(A \cap B) / \#(A \cup B)$.

Sample articles in each topic for emerging markets

\begin{tabular}{llrl}
\hline Topic & Date & Sent & Headline \\
\hline Mkt & $1997-11-06$ & -0.22 & Elbit Ltd $<$ ELBT3.TA $><$ ELBTF.O $>$ Q3 loss $\$ 0.11$ per share \\
Mkt & $1996-02-16$ & -0.22 & Uganda shilling weakens against dollar \\
Mkt & $1999-09-06$ & 0.12 & Hungarian shares open higher on Dow gains \\
Mkt & $2015-03-05$ & 0.12 & BUZZ-USD/THB eked out small gains \\
Govt & $2011-03-16$ & -0.23 & US objects to 'excessive force' in Bahrain \\
Govt & $1997-09-18$ & -0.22 & Tehran mayor rejects resignations of 12 mayors \\
Govt & $2000-06-04$ & 0.10 & Clinton says Putin can build strong, free Russia \\
Govt & $2008-04-03$ & 0.11 & Mugabe's party expects runoff, says he will win \\
Corp & $2011-01-19$ & -0.25 & BRIEF-Moody's downgrades Tunisia's to Baa3, outlook nega- \\
& & & tive \\
Corp & $2011-01-31$ & -0.25 & BRIEF-Moody's downgrades Egypt to Ba2, negative outlook \\
Corp & $2013-05-02$ & 0.14 & CORRECTED-TABLE-Philippines' sovereign credit rating his- \\
& & & tory \\
Corp & $2013-03-27$ & 0.16 & TABLE-Philippines' sovereign credit rating history \\
Comms & $2008-09-12$ & -0.13 & BP says Baku-Supsa oil pipeline remains shut \\
Comms & $1996-05-09$ & -0.12 & Russia's Novorossiisk oil port still shut by fog \\
Comms & $2006-12-27$ & 0.08 & Great Offshore buys anchor-handling tug vessel \\
Comms & $1997-06-26$ & 0.08 & Tunisia tender for 150,000 T U.S. wheat detailed \\
Macro & $1996-03-07$ & -0.12 & Hungary 1995 C/A deficit falls to $\$ 2.48$ billion \\
Macro & $2003-04-30$ & -0.11 & Turkish Jan-Feb c/a deficit jumps to $\$ 1.178$ bln \\
Macro & $2006-03-10$ & 0.00 & Sao Paulo volta a registrar inflacao no comeco de marco \\
Macro & $2012-09-11$ & 0.01 & CORRECTED-Lithuania current account surplus rises in June \\
\hline
\end{tabular}

Table 3: For each topic, we show sample articles whose topic allocation, i.e. $e_{\tau, j} / e_{j}$, is between $80 \%$ and $90 \%$. For all articles that satisfy this criteria, we show the top and bottom two articles by sentiment within each topic. The Sent column shows our sentiment measure $s_{j}$ for each article. 
Sample articles in each topic for developed markets

\begin{tabular}{|c|c|c|c|}
\hline Topic & Date & Sent & \\
\hline Mkt & $2012-05-21$ & -0.20 & $\begin{array}{l}\text { BRIEF-FINRA Panel awards John Galinsky } \$ 3.5 \mathrm{mln} \text { in com- } \\
\text { pensatory damages for breach of contract against Advanced Eq- } \\
\text { uities }\end{array}$ \\
\hline Mkt & $03-03-25$ & -0.20 & o rises above $\$ 1.07 \mathrm{a}$ \\
\hline Ikt & 96-01-18 & 0.12 & larke confiden \\
\hline Ikt & 10-11-02 & 0.12 & usly optimistic for good cl \\
\hline Govt & 2009-01-08 & -0.30 & $\begin{array}{l}\text { BRIEF-UK Serious Fraud Office to probe Madoff's UK opera- } \\
\text { tions }\end{array}$ \\
\hline ovt & & -0.25 & er-Former \\
\hline ovt & & 0.13 & UZ \\
\hline ovt & & 0.13 & UZ \\
\hline Corp & 2014 & -0.15 & ulators about Allerg: \\
\hline Corp & 6 & -0.15 & ces termination of proposed divest- \\
\hline rp & & 0 . & anga \\
\hline orp & & 0.1 & e \\
\hline omn & 17 & -0.07 & ust \\
\hline $\mathrm{m}$ & & -0.07 & op \\
\hline omms & & 0.13 & ess rel \\
\hline & & 0.13 & גו דוטה \\
\hline & & -0 . & \\
\hline & & -0.2 & $\rightarrow$ Q2 \\
\hline & & & $\begin{array}{l}\text { BRIEF-Moody's revises euramax's outlook to stable from posi- } \\
\text { tive }\end{array}$ \\
\hline Credit & 21 & 0.23 & Moody's revises Pulte's outlook to stable from posit \\
\hline
\end{tabular}

Table 4: For each topic, we show sample articles whose topic allocation, i.e. $e_{\tau, j} / e_{j}$, is between $80 \%$ and $90 \%$. For all articles that satisfy this criteria, we show the top and bottom two articles by sentiment within each topic. The Sent column shows our sentiment measure $s_{j}$ for each article. 


\section{Data definitions summary}

\begin{tabular}{|c|c|}
\hline Variable & Definition \\
\hline return & Total monthly stock returns (in \%) including capital gains and dividend yield \\
\hline return ${ }^{N}$ & Cumulative stock returns from the start of month $t$ to the end of month $t+N-1$ \\
\hline sigma & Rolling 20-day realized volatility reported in annualized terms \\
\hline drawdown $n^{N}$ & $\begin{array}{l}\text { For a } \$ 100 \text { initial investment, the maximum loss - potentially } 0 \text { - experienced } \\
\text { over the subsequent } N \text {-month period (for } 12 \text {-month drawdowns, we often omit } \\
N)\end{array}$ \\
\hline retmi & Negative portion of returns (i.e. $\max (-$ return, 0$)$ ) \\
\hline retpl & Positive portion of returns (i.e. $\max ($ return, 0$)$ ) \\
\hline value & $\begin{array}{l}\text { Average stock index level from } 4.5 \text { to } 5.5 \text { years ago divided by current index } \\
\text { level }\end{array}$ \\
\hline$g d p$ & Rate of growth of real GDP \\
\hline gdpdeflator & Rate of change of the GDP deflator \\
\hline$c p$ & Private sector credit to GDP ratio \\
\hline$d c p$ & First difference of credit to GDP ratio \\
\hline rate & $\begin{array}{l}\text { Local currency rate: deposit rate for EM and 5-10 year governemnt bond yields } \\
\text { for DM }\end{array}$ \\
\hline dexch & $\begin{array}{l}\text { Change in value of US Dollar in terms of local currency (positive values are } \\
\text { local currency depreciations), truncated at } \pm 50 \%\end{array}$ \\
\hline pre & Dummy variable set to 1 if month $t$ is 6 or fewer months prior to an election \\
\hline post & Dummy variable set to 1 if month $t$ is 6 of fewer months after an election \\
\hline entropy & Daily word count weighted average of article level $H_{j}$ averaged over a month \\
\hline artcount & Number of articles written about a country per day, averaged over a month \\
\hline$s[$ Topic $]$ & Sentiment $s_{\tau}$ in a given month due to Topic \\
\hline$f[$ Topic $]$ & Frequency $f_{\tau}$ of articles in a given month in Topic \\
\hline
\end{tabular}

Table 5: Data definitions summary. More detailed information on variable construction and data sources is available in the Appendix. Topic is one of: government, markets, macroeconomics, credit, commodities or corporate events. 


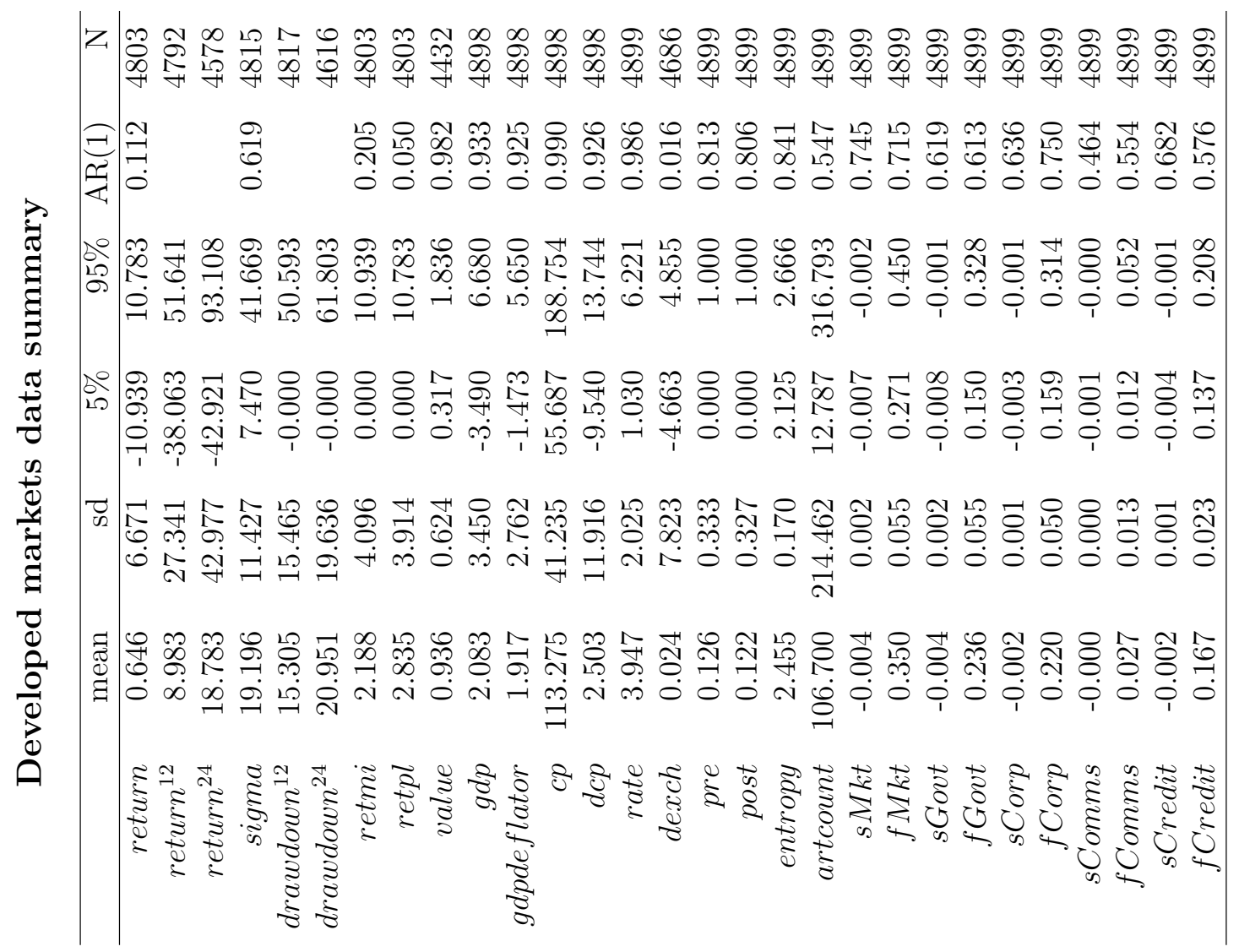

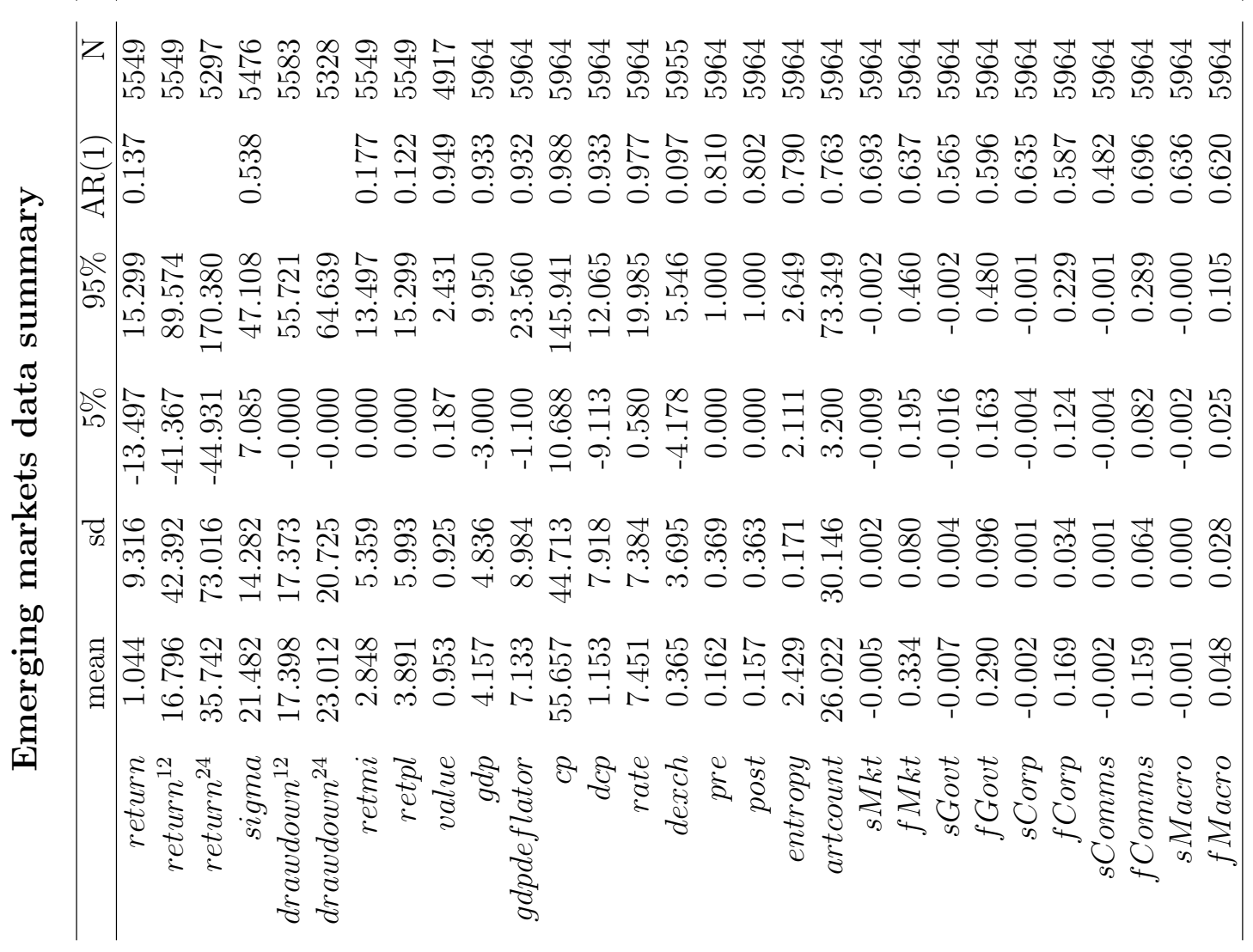

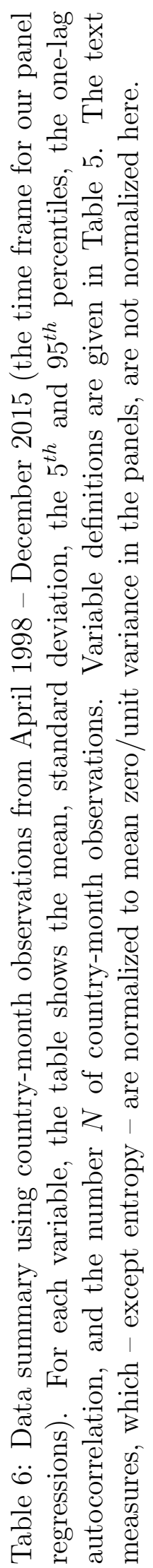




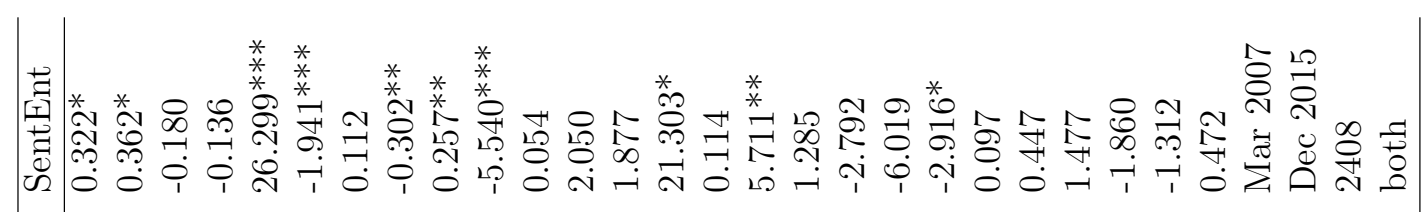

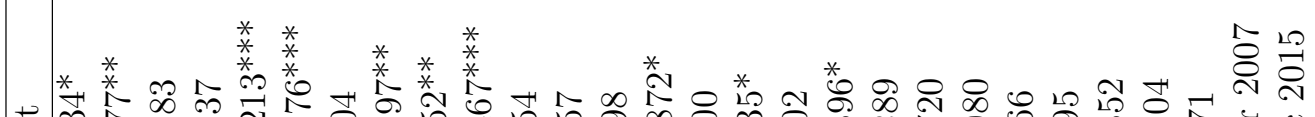

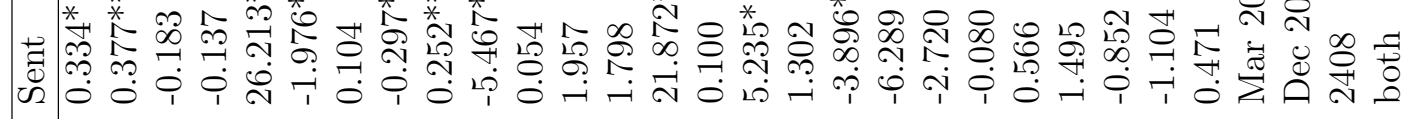

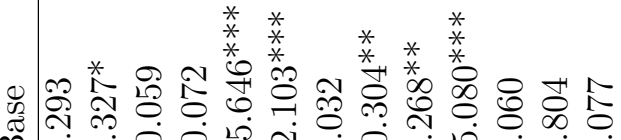

and

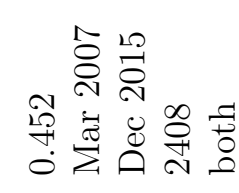

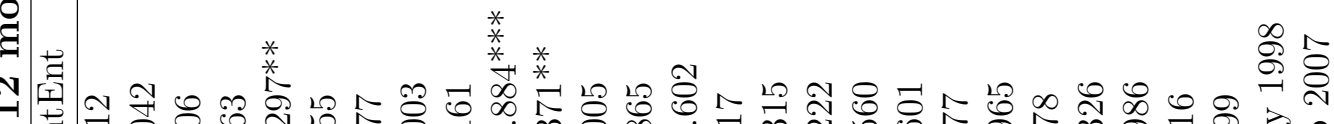

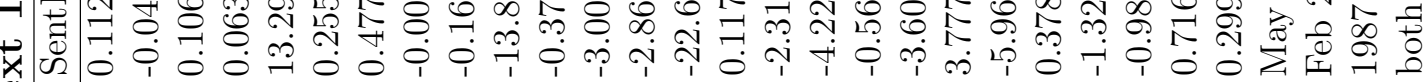

s

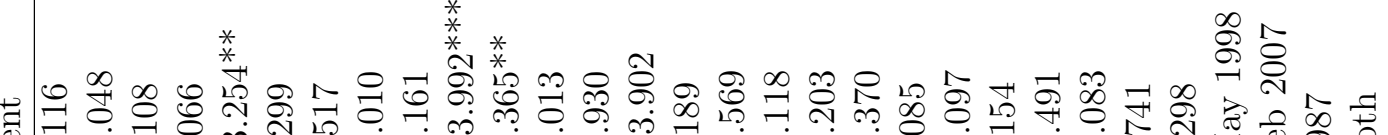

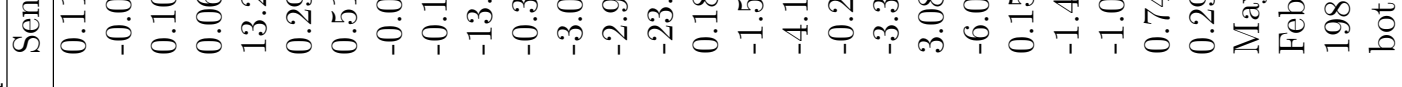

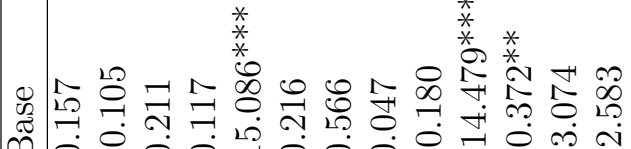

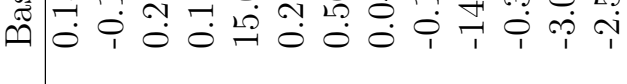

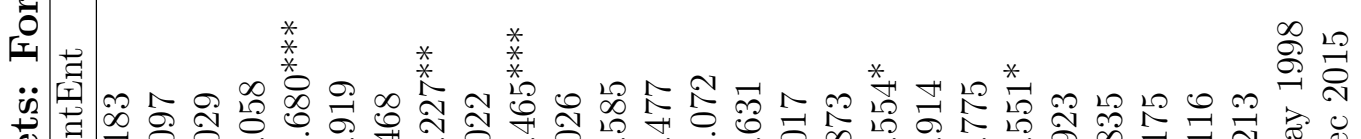

분

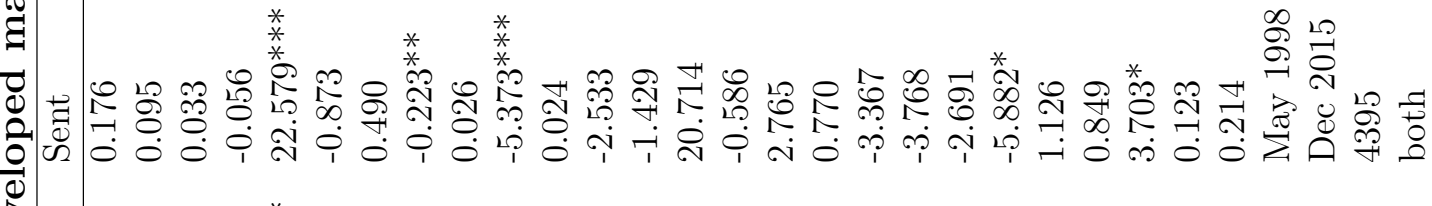

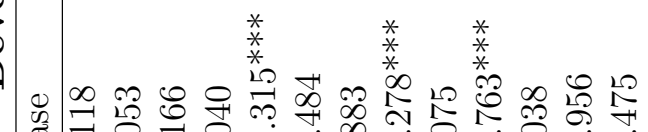

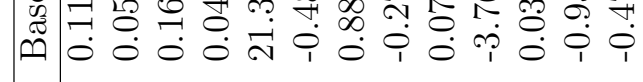

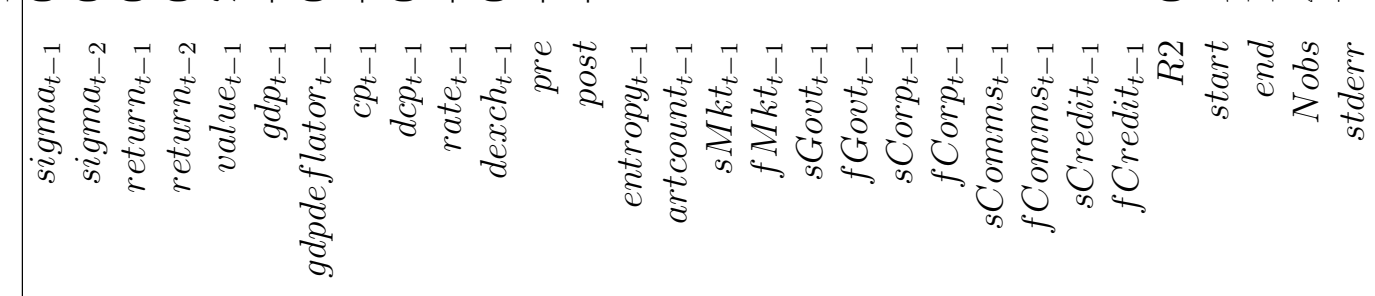

责

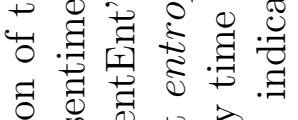
.

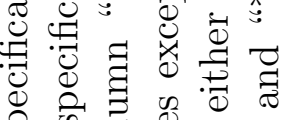
员 O

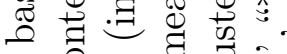

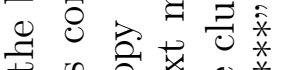

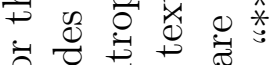

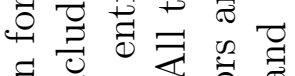

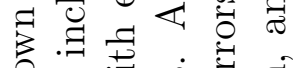
光苛完 击

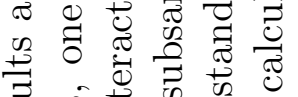
苟

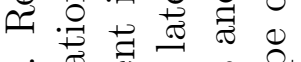

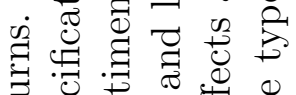

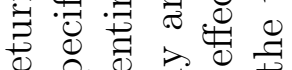

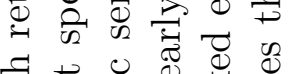

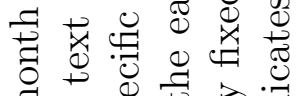

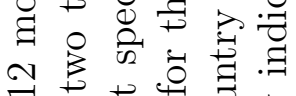

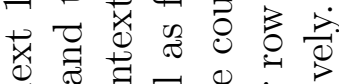
व

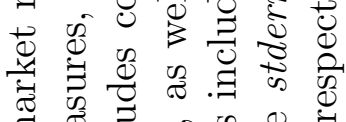

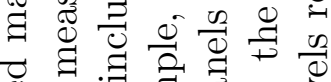

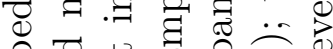

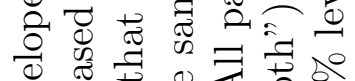

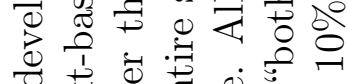

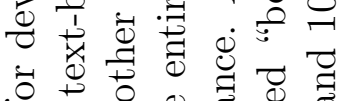
苛要

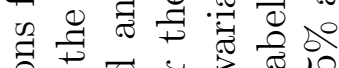

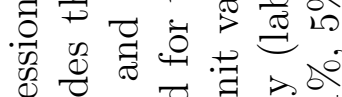

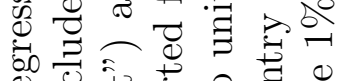

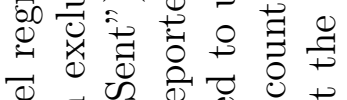
昰 ๘. N 엉

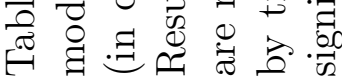




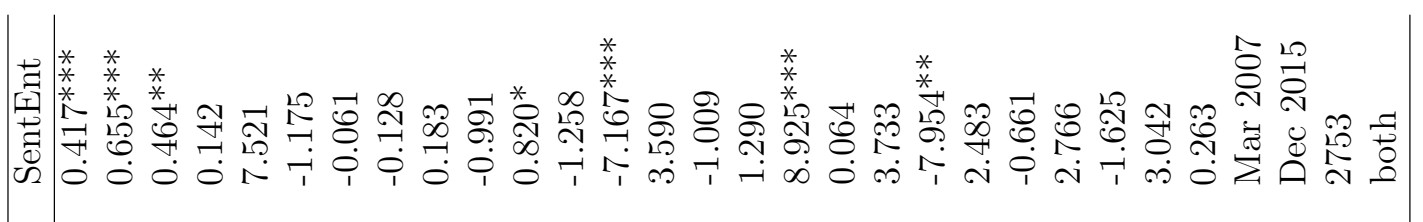

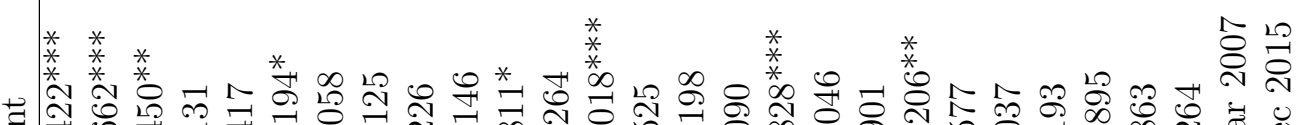

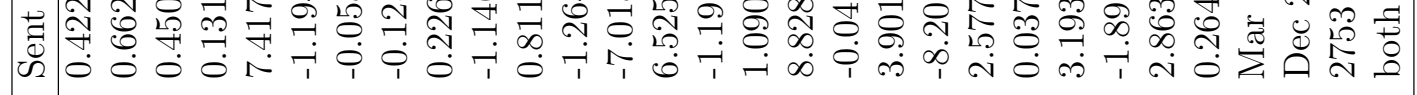

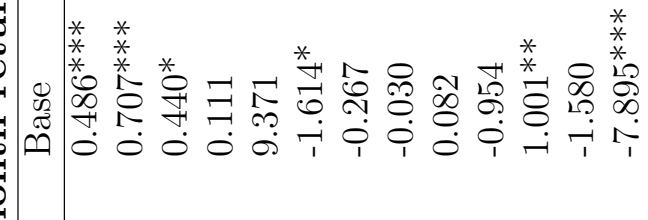

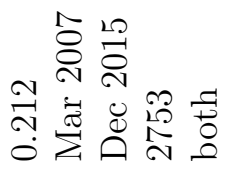

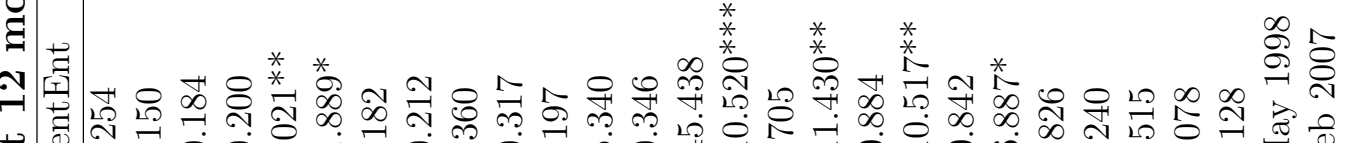

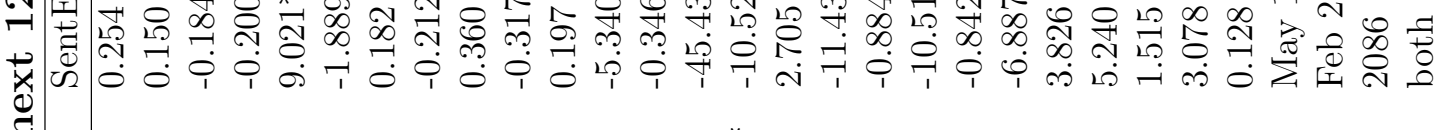

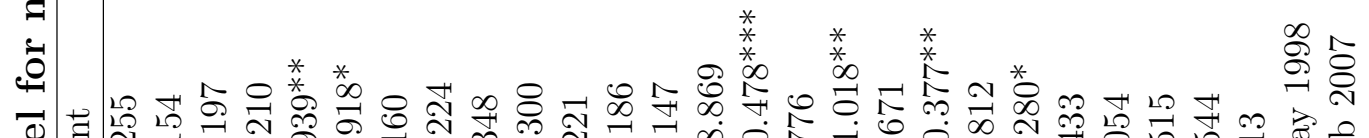

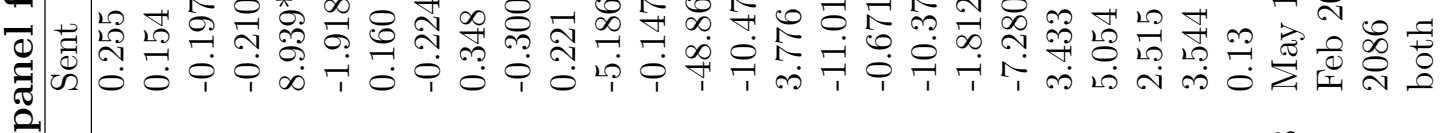

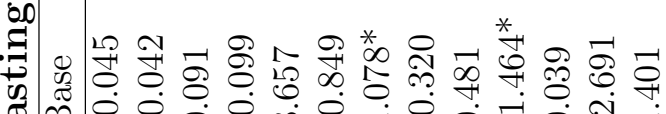

(a)

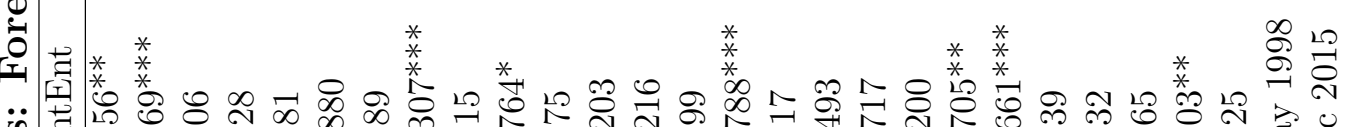

ㅂ.

胥

烍

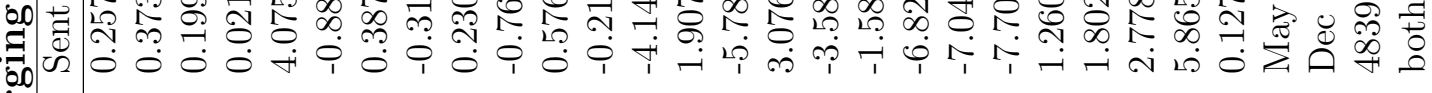

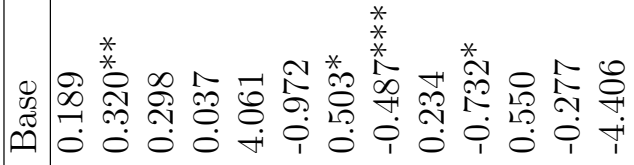

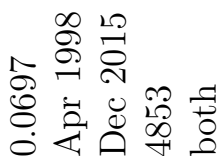

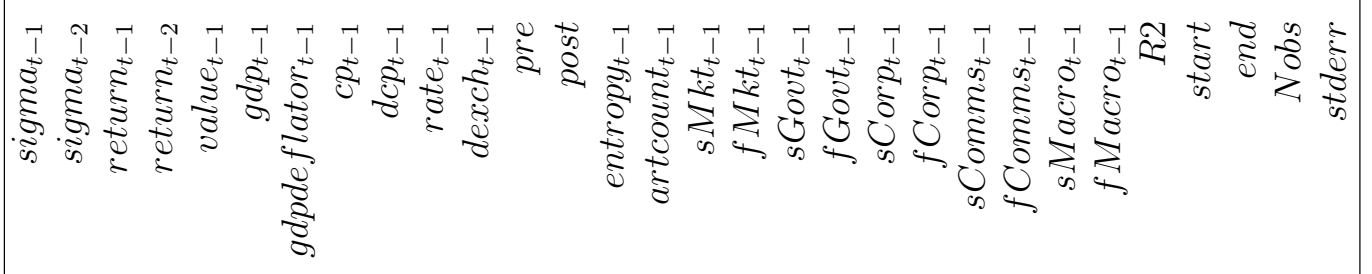




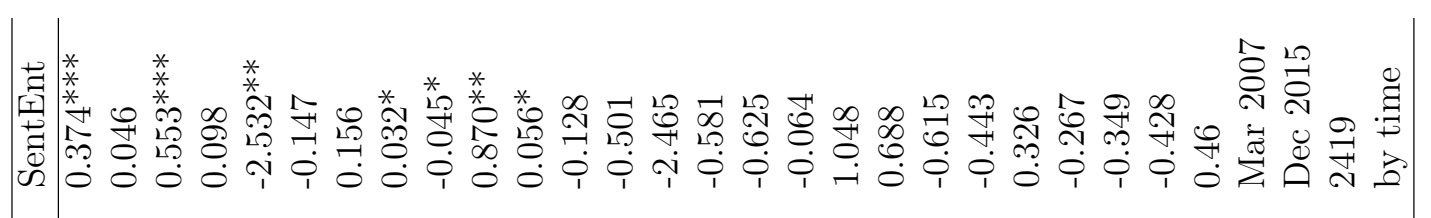

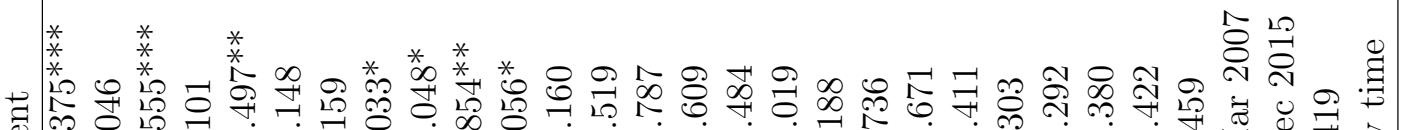
造

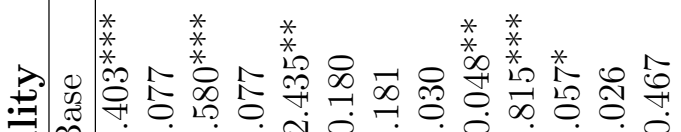

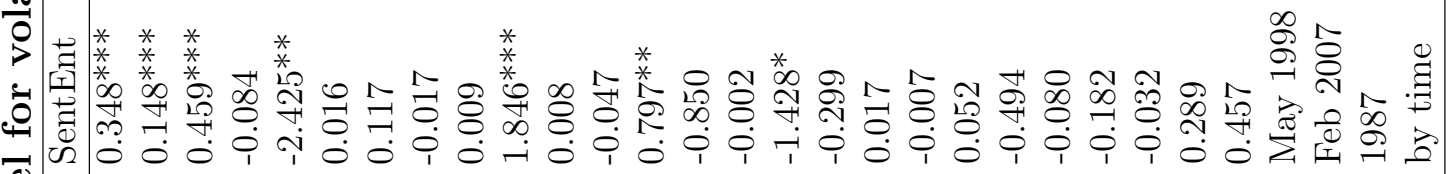

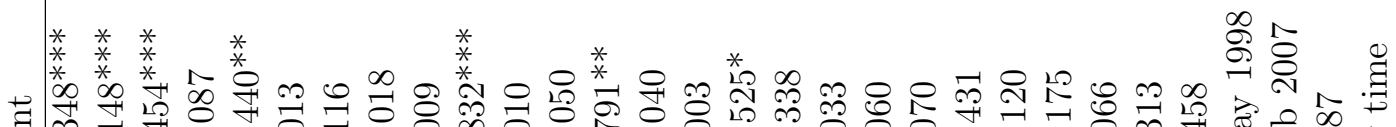

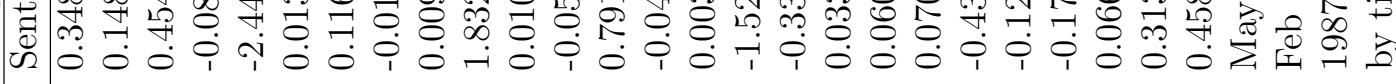

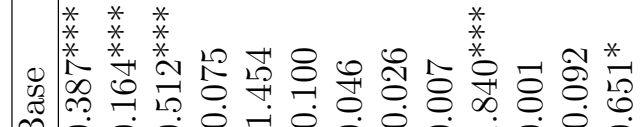

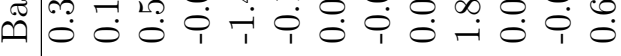

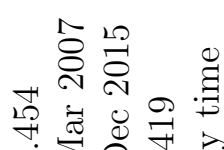

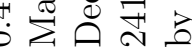

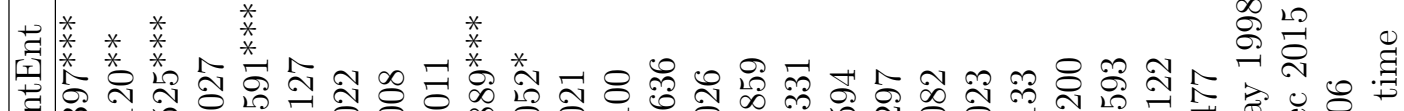

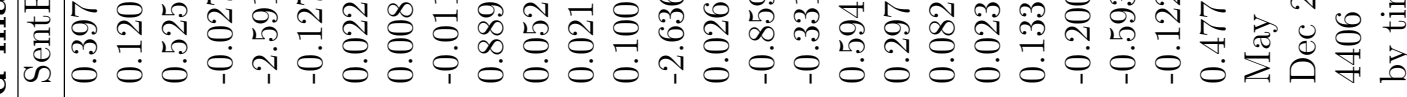

$* * * * *$

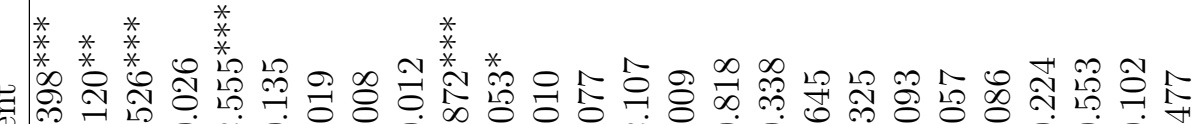

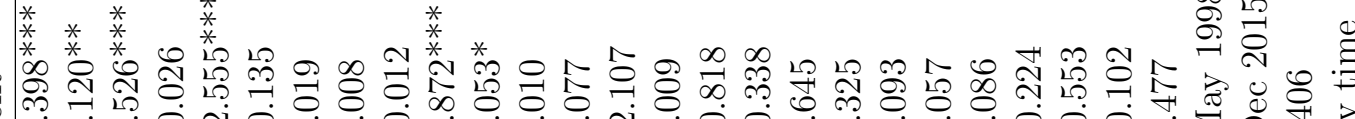

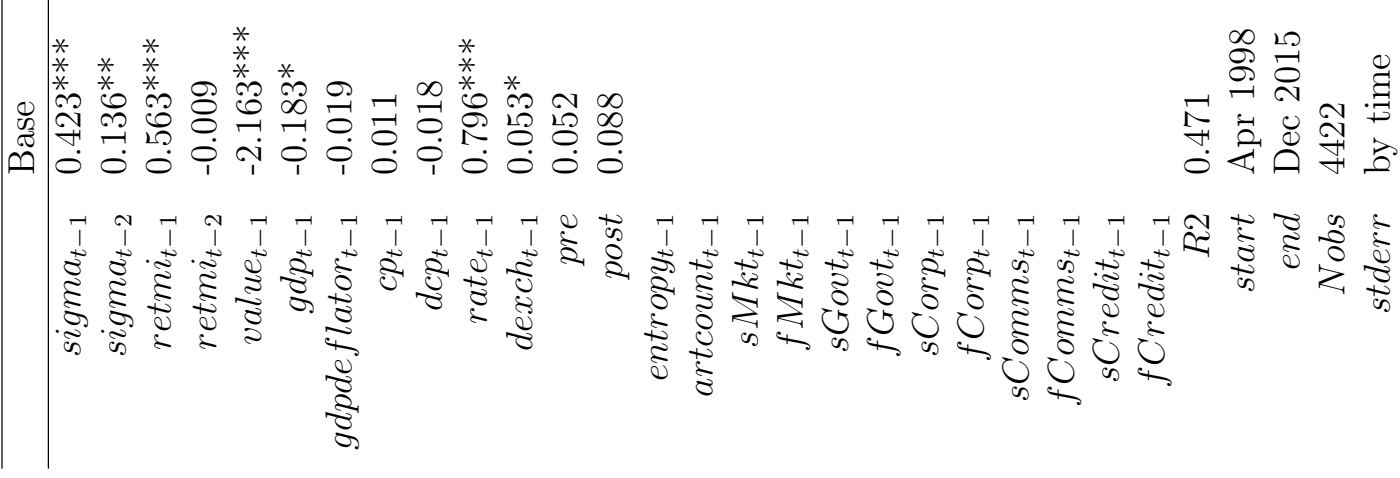

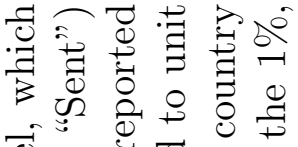

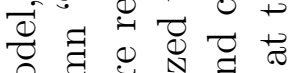

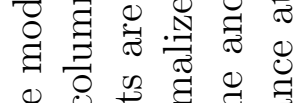

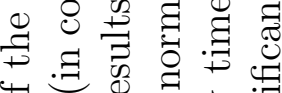
$\overrightarrow{0} \sim \overrightarrow{0}$

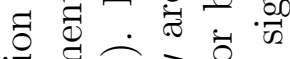
言

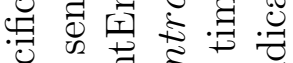

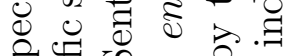
की

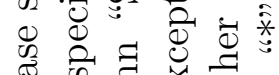

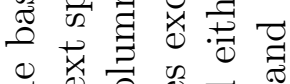
焉 के ठ․ㅇ $\exists$ के

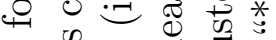
㐘完 青

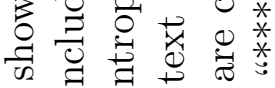

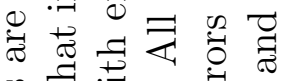

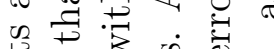

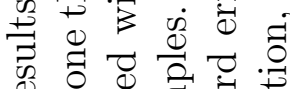

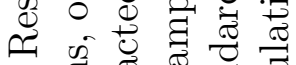

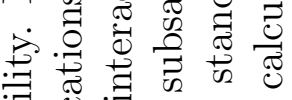

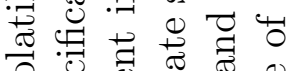
它

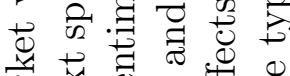

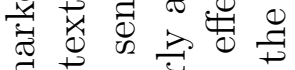
a 0 记

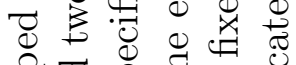

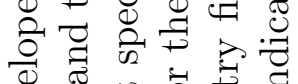

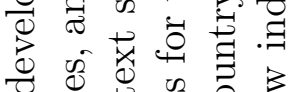
ช

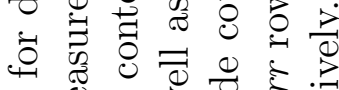
क . Uె口 o

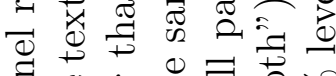

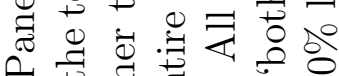
$\ddot{\theta}$ ⿹ 口 을

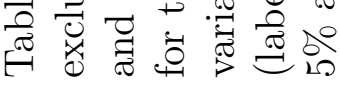




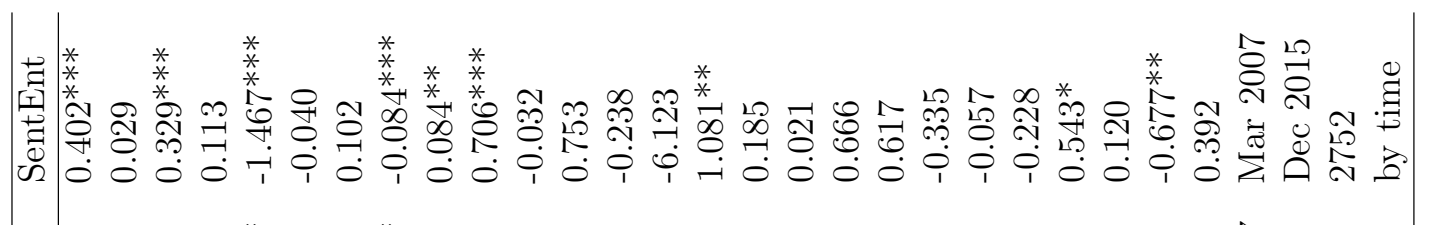

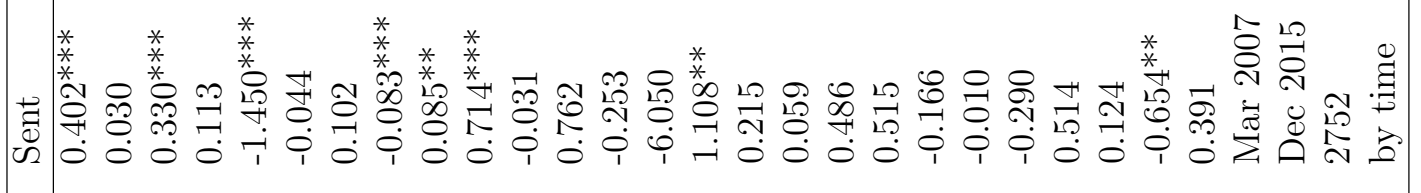

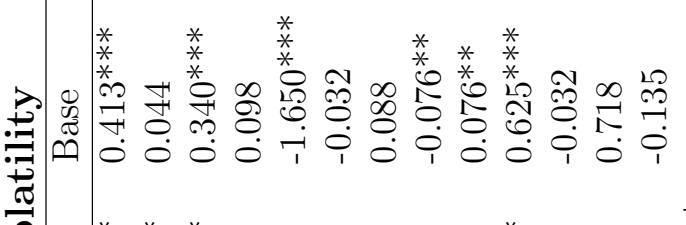

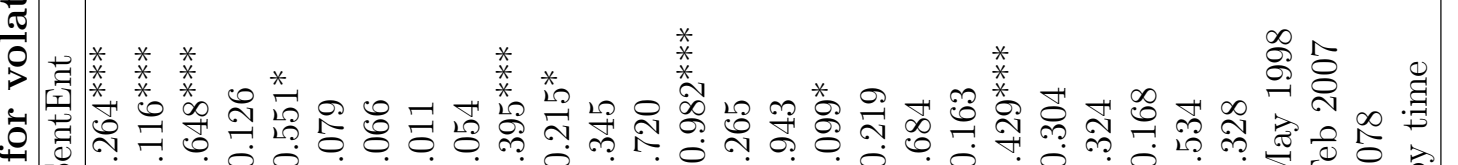

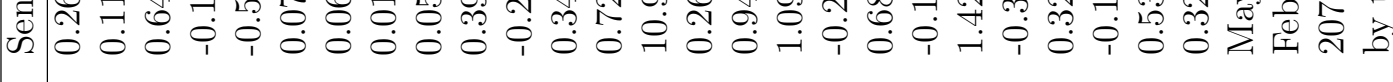

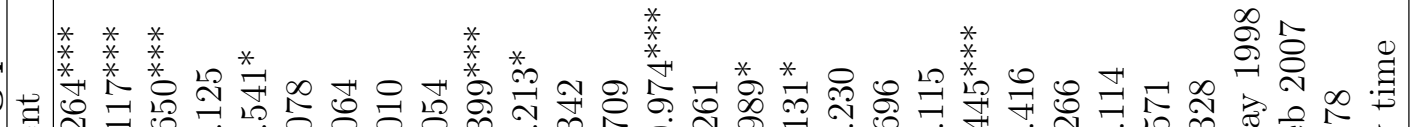

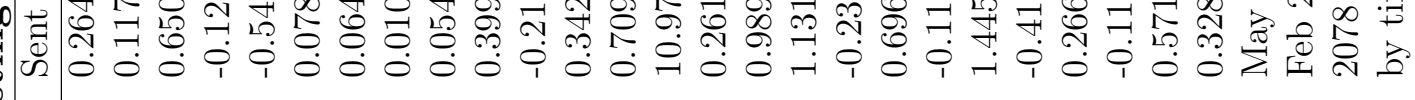

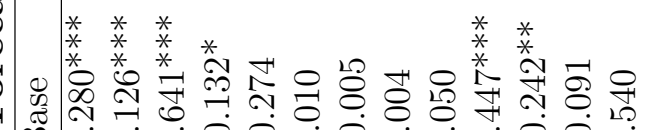

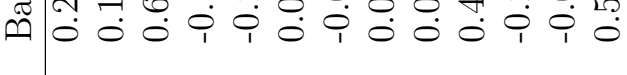

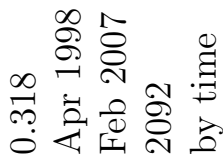

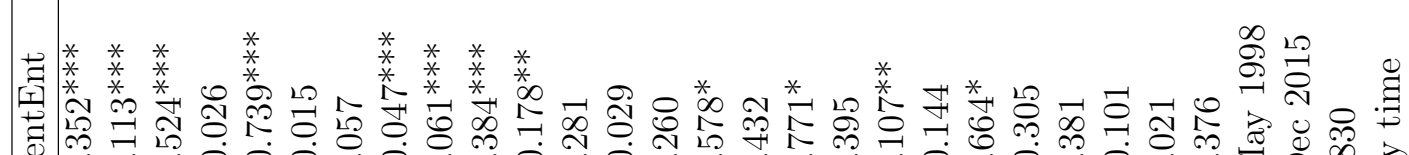

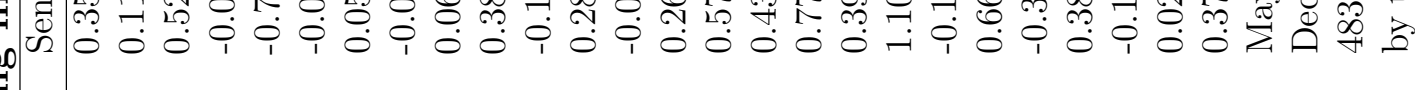

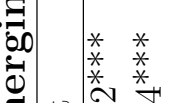

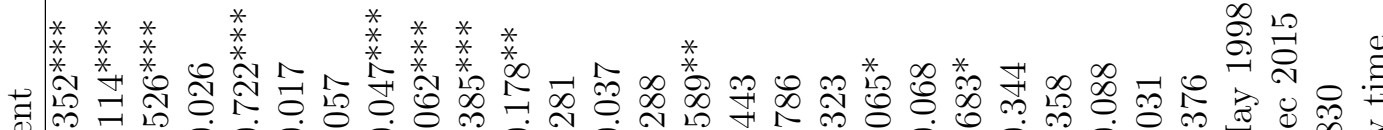

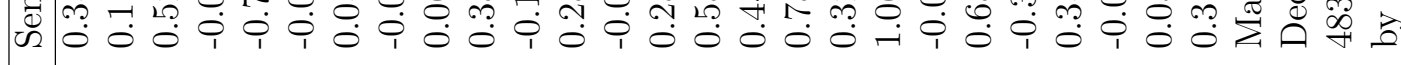

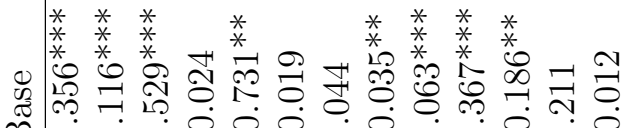

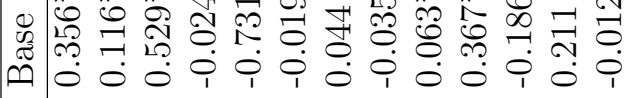

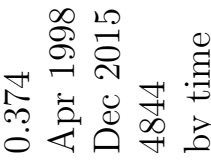

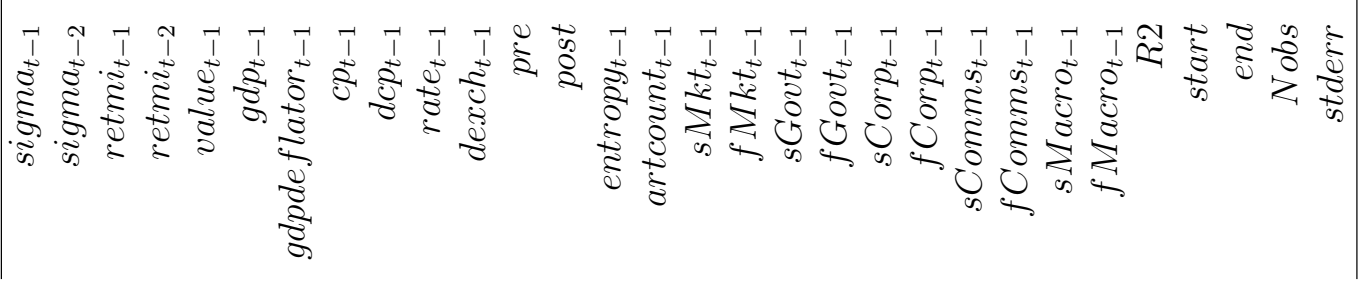




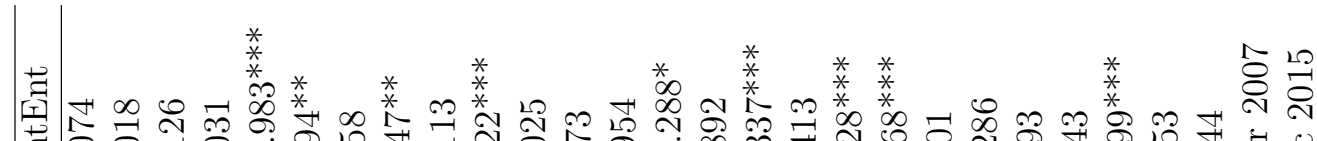

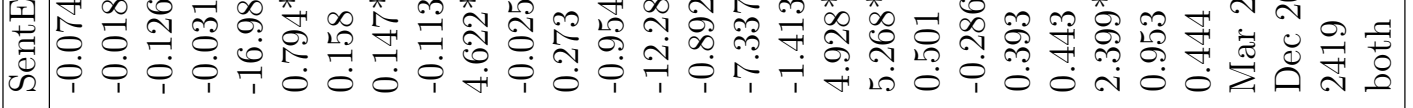

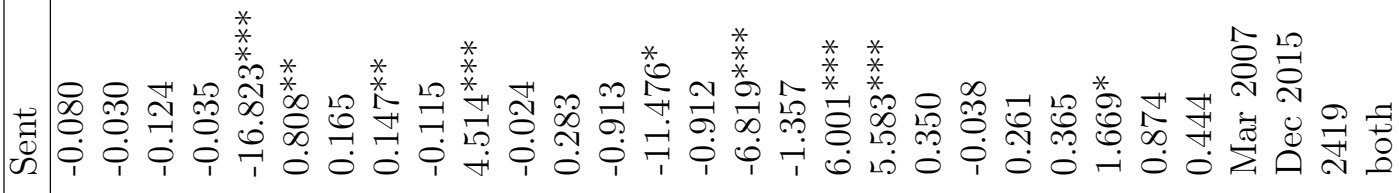

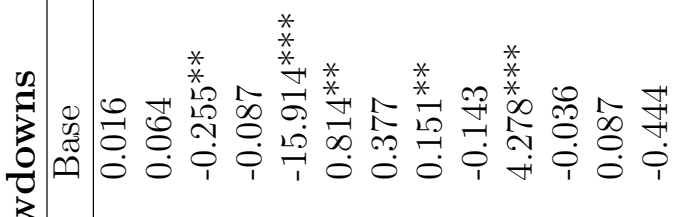

\%

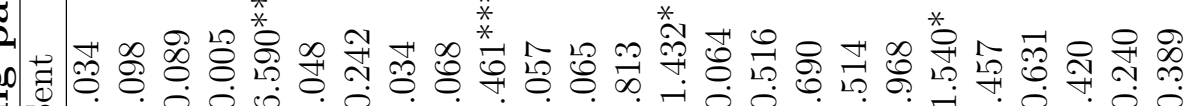

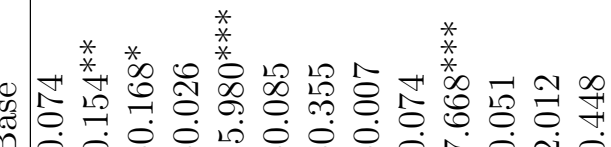

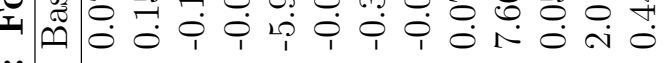

$\stackrel{\infty}{2} \stackrel{\circ}{\circ}$

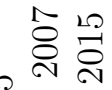

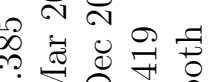

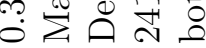

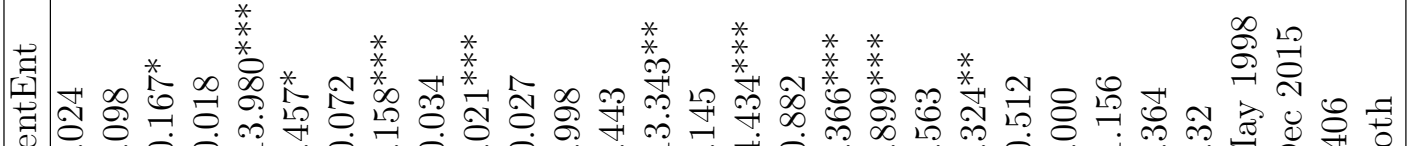

$\stackrel{\infty}{2} \stackrel{10}{\circ}$

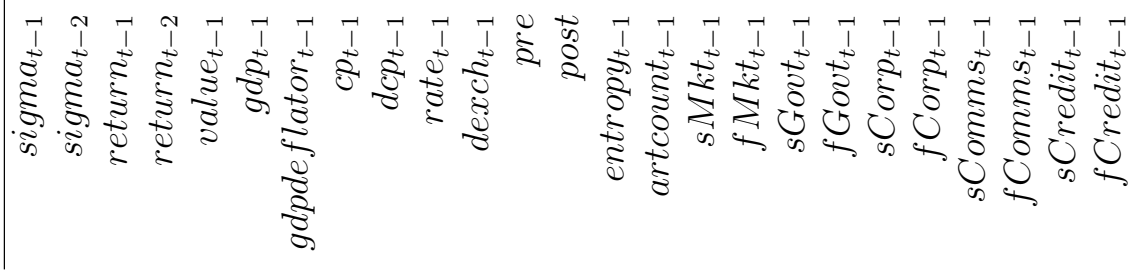

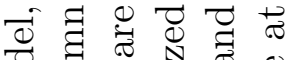
寻 号

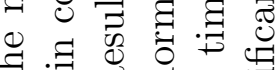

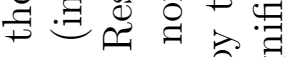

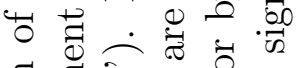

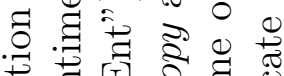
ॠ

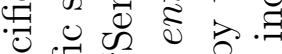
की क

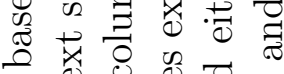

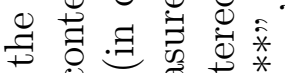

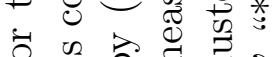

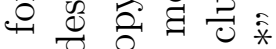

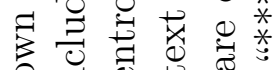
灵.

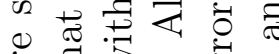
ठี 足 के की

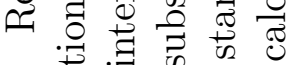
कं

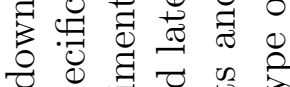

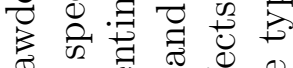

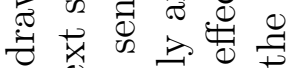
$\checkmark \stackrel{0}{0}$

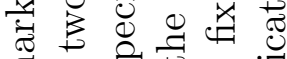
घ ठ

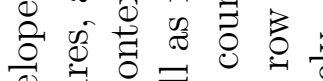

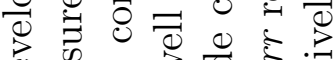

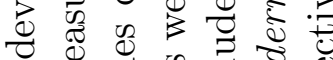
- द्व ठ

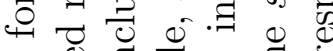

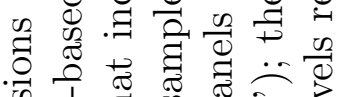

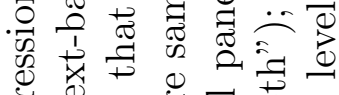

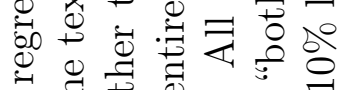
¿ శ్

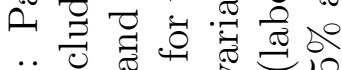
$\ddot{\ddots} \stackrel{x}{\approx}$

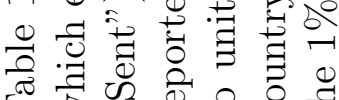
윱 


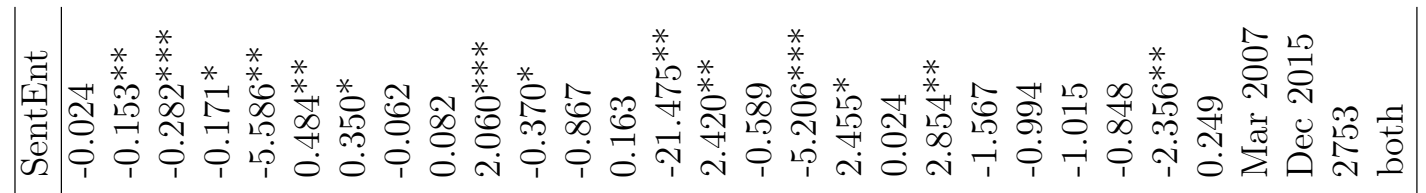

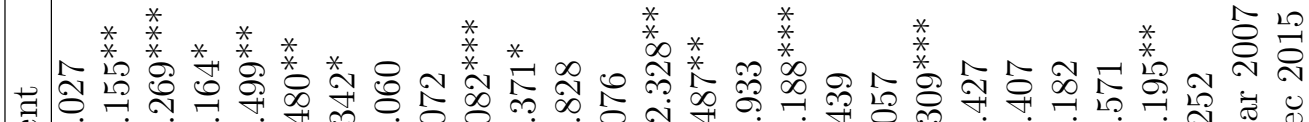

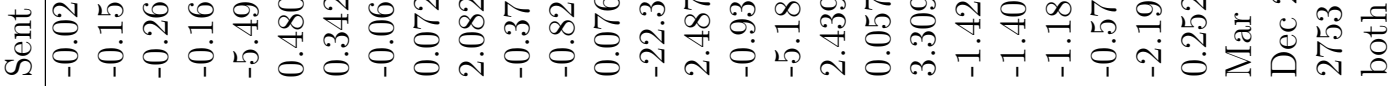

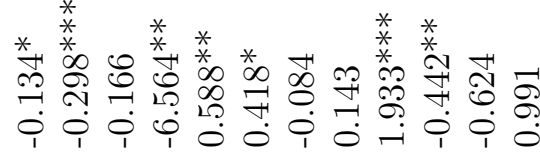

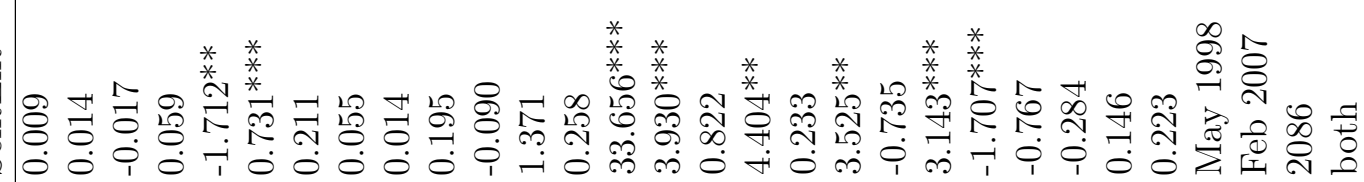

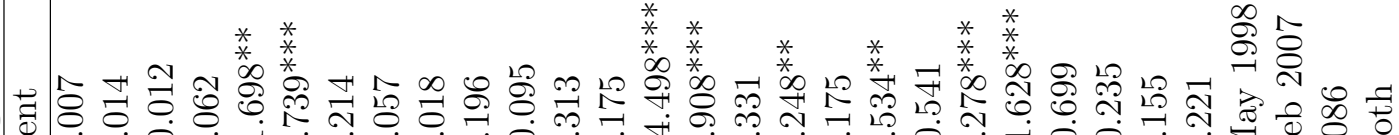

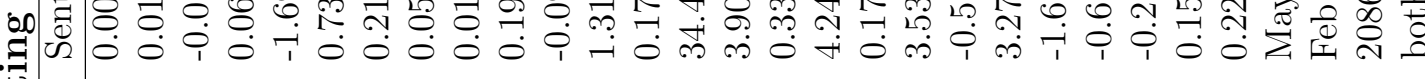

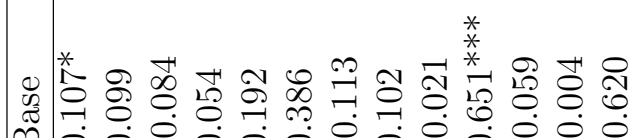

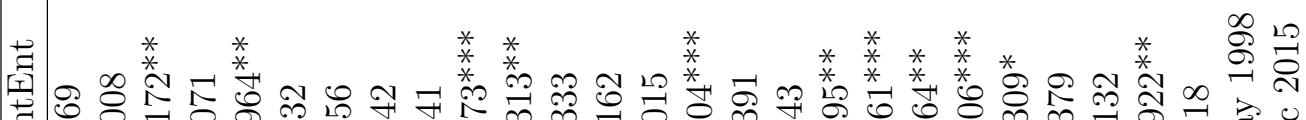

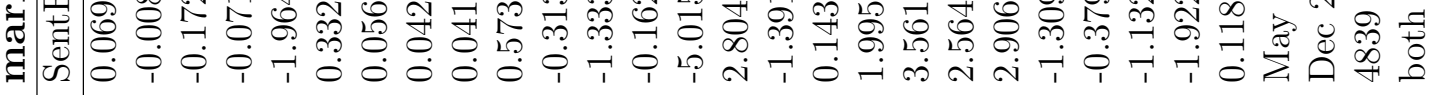

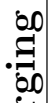

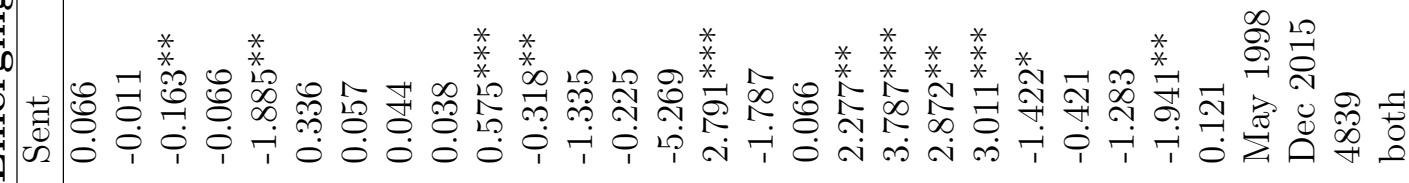

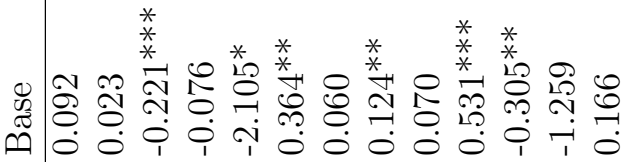

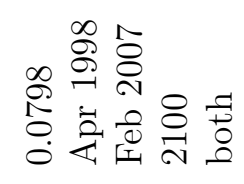

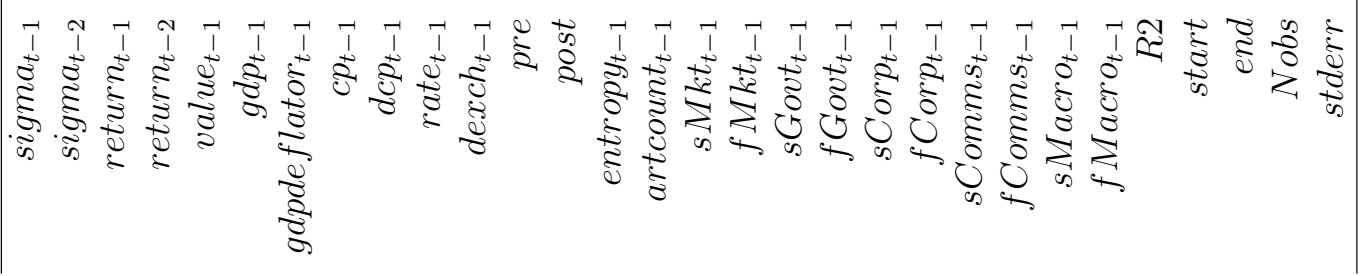

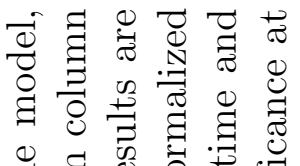

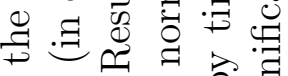

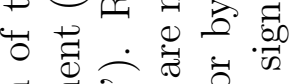

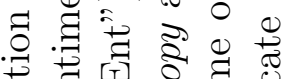

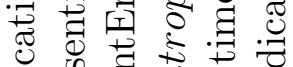

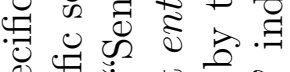
解 $\exists$ है

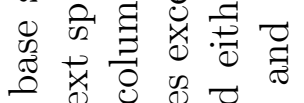
(1) 0 巳

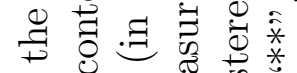

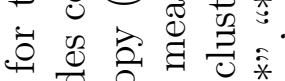

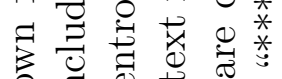

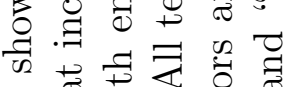

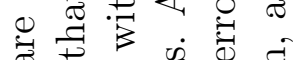

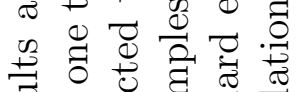
苟

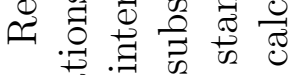
में

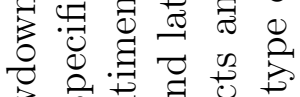

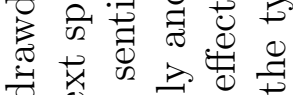

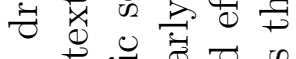

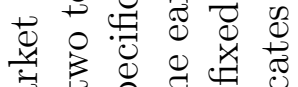

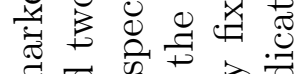

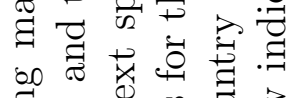
.

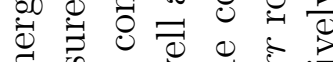
द्व के

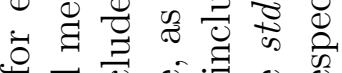
車

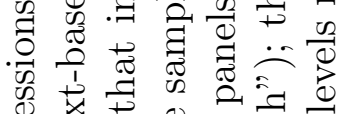

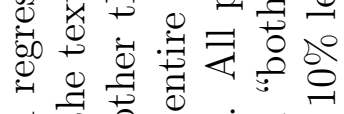

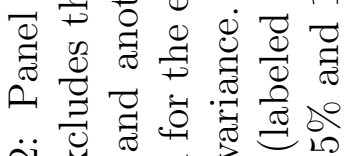

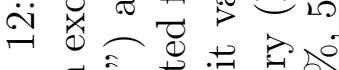

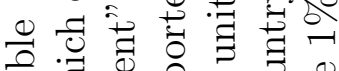
일 


\begin{tabular}{|c|c|c|c|c|}
\hline & Base EM & Full EM & Base DM & Full DM \\
\hline$g d p_{t-1}$ & $-0.028 * * *$ & -0.009 & $-0.081^{* * *}$ & $-0.029^{* * *}$ \\
\hline gdpdeflator ${ }_{t-1}$ & -0.005 & -0.006 & $-0.030 * *$ & -0.015 \\
\hline$c p_{t-1}$ & $0.014^{* * *}$ & $0.010^{* * *}$ & 0.001 & -0.001 \\
\hline$d c p_{t-1}$ & -0.004 & -0.005 & $-0.008 * * *$ & $-0.004^{* * *}$ \\
\hline rate $_{t-1}$ & 0.007 & 0.002 & $-0.188 * * *$ & $-0.226 * * *$ \\
\hline $\operatorname{dexch}_{t-1}$ & $0.033^{* * *}$ & 0.016 & -0.002 & $-0.003^{*}$ \\
\hline pre & $0.131^{* *}$ & $0.119^{*}$ & 0.024 & 0.036 \\
\hline post & $0.211^{* * *}$ & $0.171^{* *}$ & $0.162^{* * *}$ & $0.096^{*}$ \\
\hline entropy $_{t-1}$ & & $-1.236^{* * *}$ & & -0.336 \\
\hline artcount $_{t-1}$ & & $0.106^{* * *}$ & & 0.009 \\
\hline$s M k t_{t-1}$ & & $-0.164^{* * *}$ & & $-0.331^{* * *}$ \\
\hline$f M k t_{t-1}$ & & $-0.137^{* *}$ & & 0.034 \\
\hline$s_{\text {Govt }}{ }_{t-1}$ & & -0.069 & & $0.137^{* *}$ \\
\hline$f G o v t_{t-1}$ & & -0.013 & & $0.198 * *$ \\
\hline$s \operatorname{Corp}_{t-1}$ & & -0.063 & & $0.165^{* * *}$ \\
\hline$f C o r p_{t-1}$ & & 0.049 & & $0.194^{* * *}$ \\
\hline$s$ Comms $_{t-1}$ & & -0.036 & & -0.020 \\
\hline fComms $_{t-1}$ & & 0.028 & & -0.006 \\
\hline$s$ Macro $_{t-1}$ & & -0.059 & & \\
\hline$f M a c r o_{t-1}$ & & $-0.151^{* * *}$ & & \\
\hline${ }_{s}$ Credit $_{t-1}$ & & & & $-0.327 * * *$ \\
\hline fCredit $_{t-1}$ & & & & 0.016 \\
\hline$R 2$ & 0.118 & 0.227 & 0.136 & 0.336 \\
\hline start & Apr 1998 & May 1998 & Apr 1998 & May 1998 \\
\hline end & Dec 2015 & Dec 2015 & Dec 2015 & Dec 2015 \\
\hline Nobs & 1220 & 1215 & 2249 & 2240 \\
\hline stderr & by time & by time & by time & by time \\
\hline
\end{tabular}

Table 13: Regression of the BBD country-level policy uncertainty measures at time $t$ on one-month lags of macro control and one-month lags of our text measures. Results are shown for the EM sample (Brazil, Chile, China, India, Russia, and South Korea) and the DM sample (USA, Canada, Germany, UK, Italy, France, Spain, Netherlands, Japan, Australia, and Ireland). All text measures except entropy are normalized to unit variance. All panels include country fixed effects and standard errors are clustered either by time or by time and country (labeled "both"); the stderr row indicates the type of calculation, and "***", "**", and "**" indicate significance at the 1\%, 5\% and $10 \%$ levels respectively. 


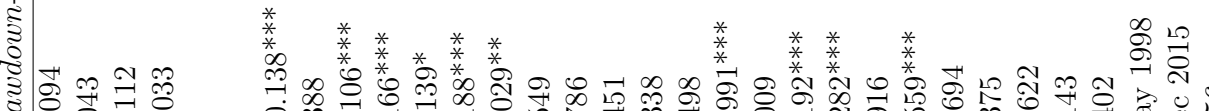

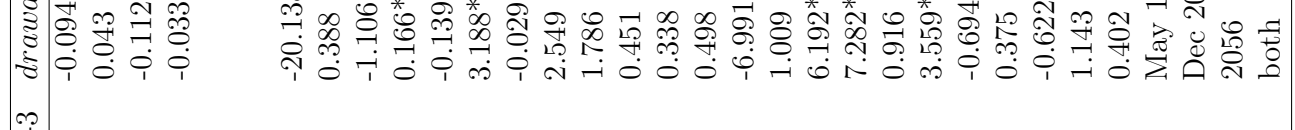

量

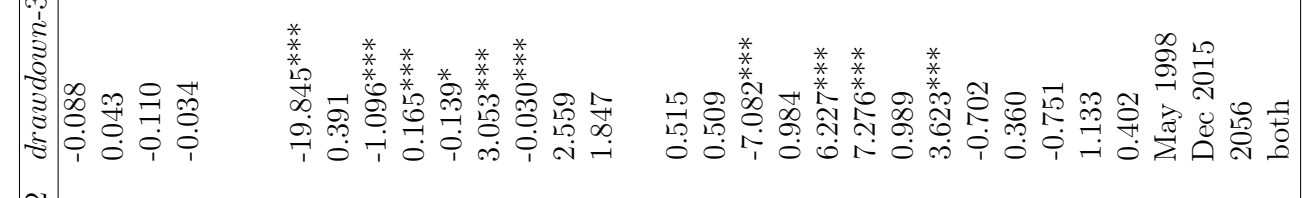

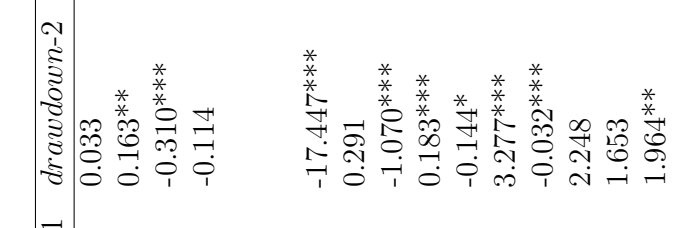

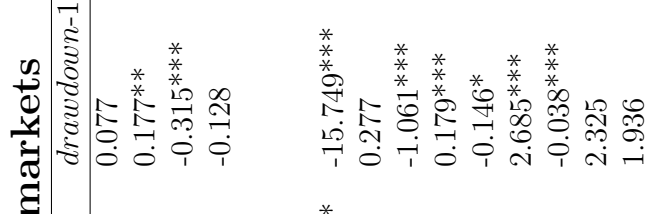

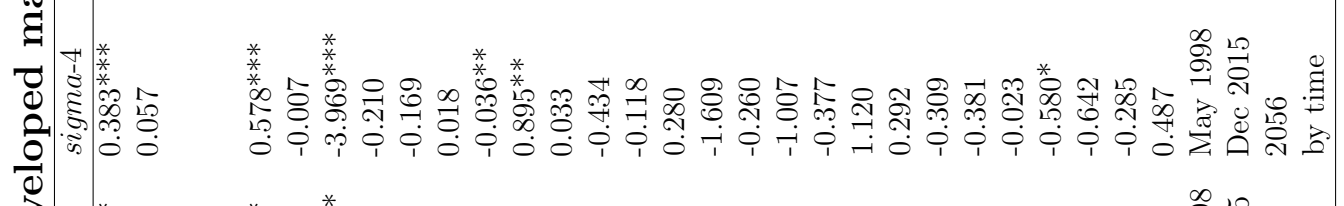

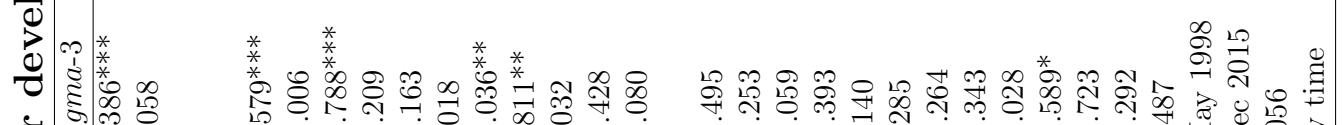

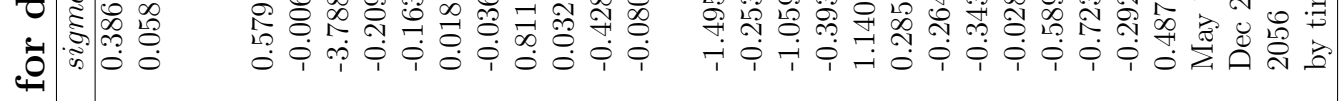

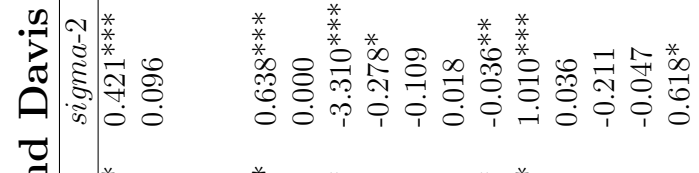

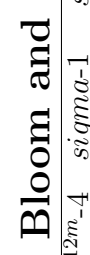

:

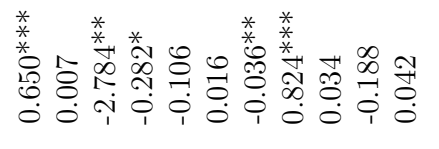

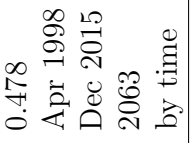

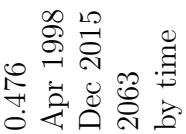

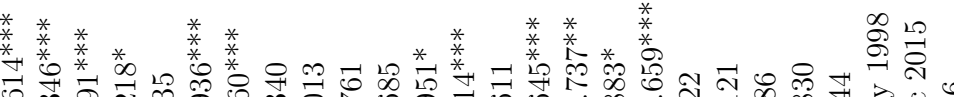

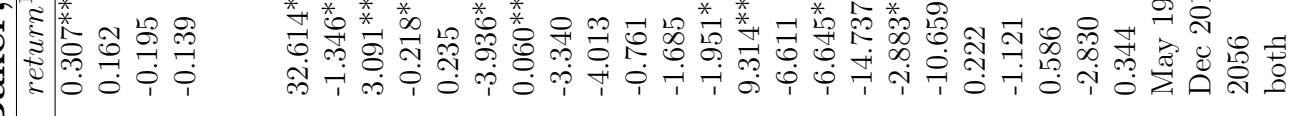

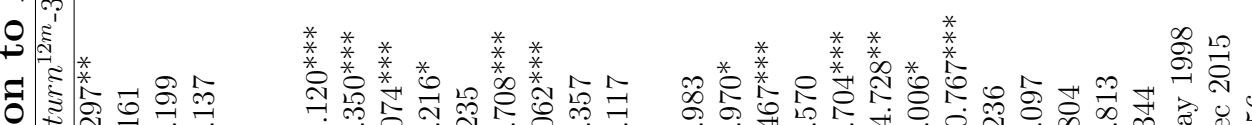

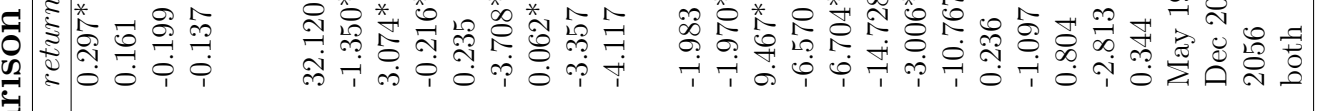

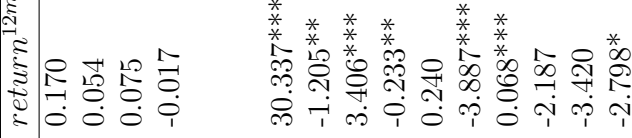

I

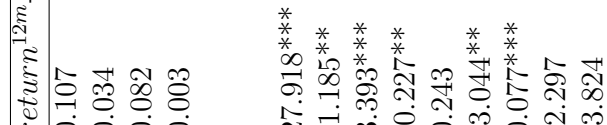

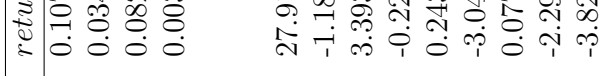

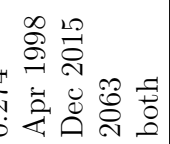

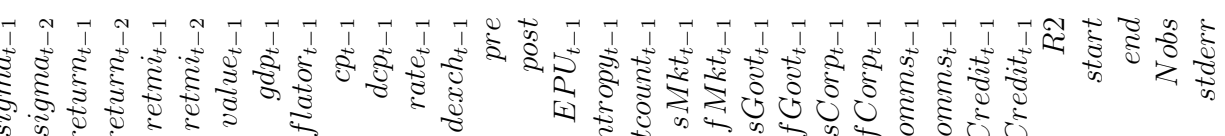

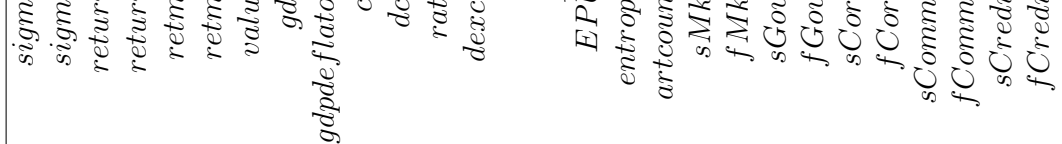

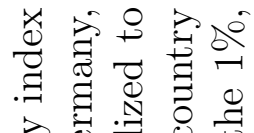

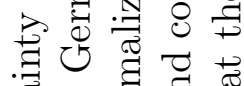

芯蕗

㱐进

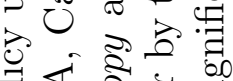

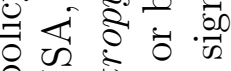

คิ:

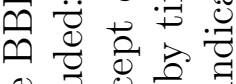

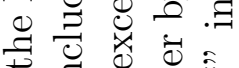

0.

荷.

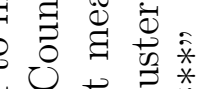

ర 氮

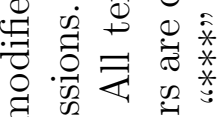

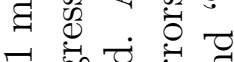

구유

ซี

$\sigma \cdot \overrightarrow{0}$

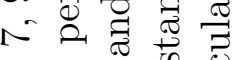

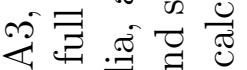

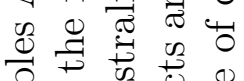

Е

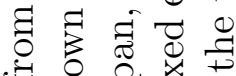

4 월

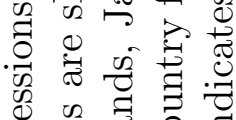

ర్⿻

త

范艺氙 है

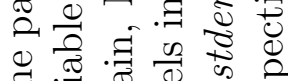

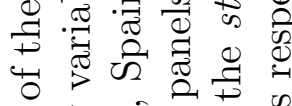

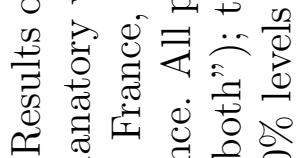

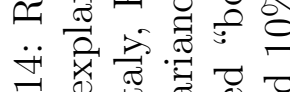

둘

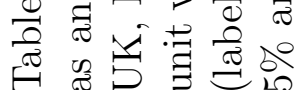




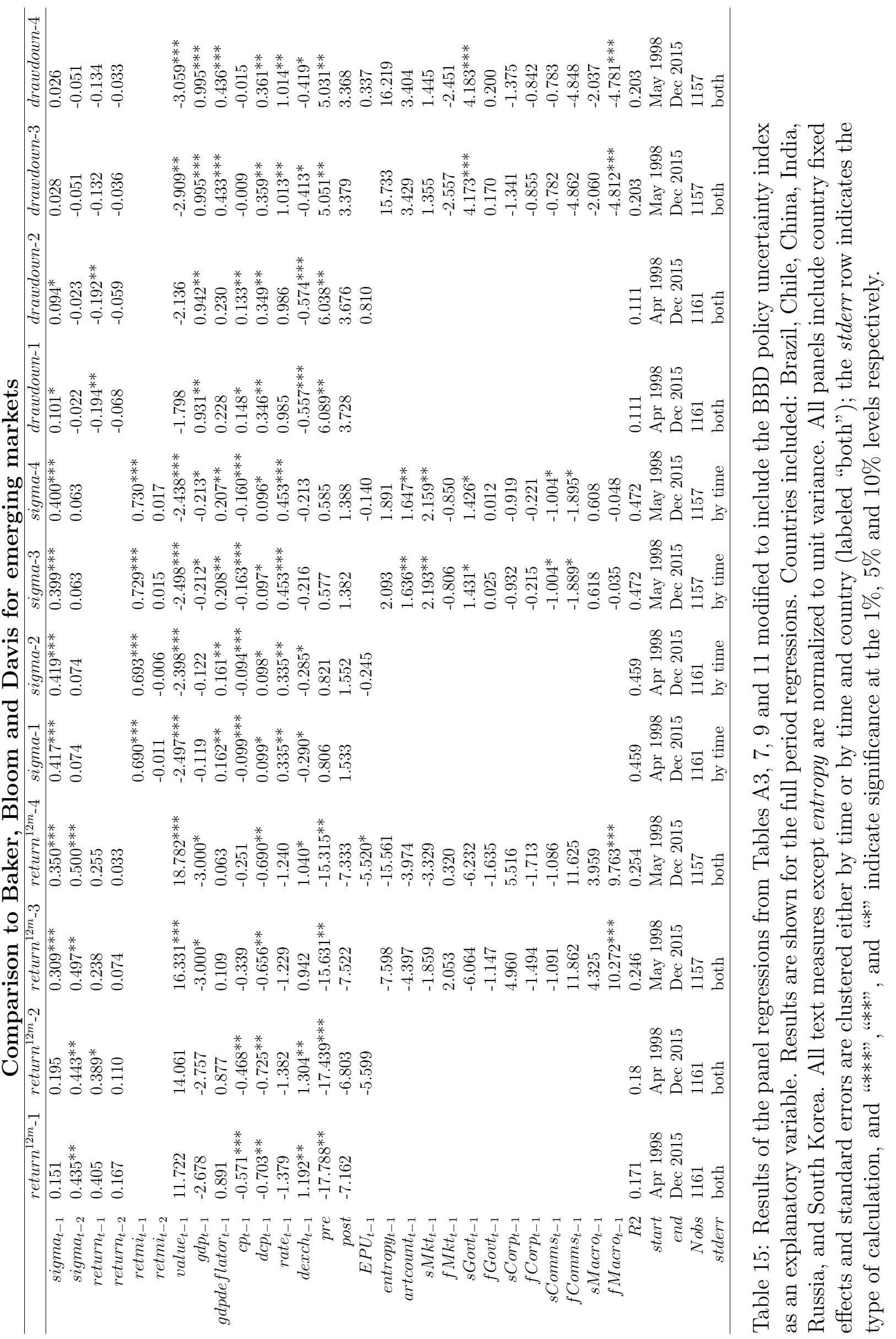




\section{$\underline{\text { Panel A }}$}

Developed market strategy

\begin{tabular}{lllll}
\hline Model & Alpha & Mkt.RF & fxcarry & fxusd \\
\hline CM & 8.816 & 0.443 & -0.168 & -0.413 \\
& $(1.438)$ & $(2.702)$ & $(-0.591)$ & $(-2.756)$ \\
Base & 6.809 & 0.570 & -0.076 & -0.395 \\
& $(1.380)$ & $(4.286)$ & $(-0.347)$ & $(-2.784)$ \\
Naive & -2.765 & 0.666 & 0.123 & -0.024 \\
& $(-0.678)$ & $(4.635)$ & $(0.749)$ & $(-0.219)$ \\
\hline
\end{tabular}

Emerging market strategy

\begin{tabular}{lllll}
\hline Model & Alpha & Mkt.RF & fxcarry & fxusd \\
\hline CM & 8.801 & 0.358 & 0.103 & -0.298 \\
& $(1.960)$ & $(2.621)$ & $(0.591)$ & $(-2.235)$ \\
Base & 3.271 & 0.499 & 0.137 & -0.318 \\
& $(0.892)$ & $(4.677)$ & $(1.158)$ & $(-2.645)$ \\
Naive & 2.347 & 0.529 & 0.334 & -0.175 \\
& $(0.780)$ & $(5.370)$ & $(2.281)$ & $(-1.766)$ \\
\hline
\end{tabular}

$\underline{\text { Panel B }}$

Tests comparing alphas of CM and Base models

\begin{tabular}{lccc}
\hline & Difference & \multicolumn{2}{c}{ T-test $\mathrm{p}$-values } \\
Market & in alphas $/ \mathrm{yr}$ & 2-sided & 1-sided \\
\hline DM & 2.01 & 0.082 & 0.041 \\
EM & 5.53 & 0.002 & 0.001 \\
\hline
\end{tabular}

Table 16: Panel A shows coefficients estimates from the LRV 3-factor model estimated with monthly returns. Alphas are reported annualized in percent. T-statistics are shown in parentheses. Panel B shows t-tests of the differences of alphas between the CM and Base models. All standard errors in both panels are calculated using Newey-West with auto lag selection.

The three forecasting models are: Naive which uses only in sample country fixed effects as the forecasting variables; Base which includes lagged macroeconomic and lagged market variables as the regressors; and $C M$ which includes country specific article counts, entropy, sentiment and frequency measures in addition to the variables from the Base model. Forecasts come from rolling elastic net regressions. The elastic net regressions are run over rolling 60-month windows, with weighting parameter chosen to minimize cross-validation error. The penalty is 0.75 elastic net and 0.25 ridge regression. 